Electronic Supporting Information for:

\title{
Mono-, Di- and Trinuclear Iron Complexes Featuring a New Monoanionic SNS Thiolate Ligand
}

Uttam K. Das, ${ }^{\dagger}$ Stephanie L. Daifuku, ${ }^{\dagger}$ Serge I. Gorelsky, ${ }^{\dagger}$ Ilia Korobkov, ${ }^{\dagger}$ Michael L. Neidig, ${ }^{\ddagger}$ Jennifer J. Le Roy, ${ }^{\dagger}$ Muralee Murugesu ${ }^{\dagger}$ and R. Tom Baker*, ${ }^{\dagger}$

${ }^{\dagger}$ Department of Chemistry and Biomolecular Sciences and Centre for Catalysis Research and Innovation, University of Ottawa, Ottawa, ON K1N 6N5, Canada

$\$$ Department of Chemistry, University of Rochester, Rochester, NY 14627, USA

\section{$\underline{\text { Page }}$ Table of Contents}

S4 $\quad$ Figure S1. ${ }^{1} \mathrm{H}$ NMR spectrum $\left(300 \mathrm{MHz}, \mathrm{CD}_{3} \mathrm{CN}\right)$ of $\left[\mathrm{S}^{M e} \mathrm{~N}^{H} \mathrm{~S}\right]$ ligand

S5 Figure S2. ${ }^{13} \mathrm{C}\left\{{ }^{1} \mathrm{H}\right\} \mathrm{NMR}$ spectrum $\left(101 \mathrm{MHz}, \mathrm{CD}_{3} \mathrm{CN}\right)$ of $\left[\mathrm{S}^{M e} \mathrm{~N}^{H} \mathrm{~S}\right]$ ligand

S6 Figure S3. UV-vis spectrum of $\left[\mathrm{S}^{M e} \mathrm{~N}^{H} \mathrm{~S}\right]$ ligand

S7 Figure S4. High-Resolution EI-MS spectrum of $\left[\mathrm{S}^{M e} \mathrm{~N}^{H} \mathrm{~S}\right]$ ligand

S8 Figure S5. ${ }^{1} \mathrm{H}$ NMR spectrum $\left(300 \mathrm{MHz}, \mathrm{CD}_{2} \mathrm{Cl}_{2}\right)$ of $\left[\mathrm{Fe}_{3}\left(\mu_{2}-\mathrm{S}^{M e} \mathrm{NS}^{-}\right)_{4}\right](\mathrm{OTf})_{2}(\mathbf{1})$

S9 Figure S6. ${ }^{19} \mathrm{~F}$ NMR spectrum $\left(282 \mathrm{MHz}, \mathrm{CD}_{2} \mathrm{Cl}_{2}\right)$ of $\left[\mathrm{Fe}_{3}\left(\mu_{2}-\mathrm{S}^{\mathrm{Me}} \mathrm{NS}^{-}\right)_{4}\right](\mathrm{OTf})_{2}(\mathbf{1})$

S10 Figure S7. UV-vis spectrum of $\left[\mathrm{Fe}_{3}\left(\mu_{2}-\mathrm{S}^{M e} \mathrm{NS}^{-}\right)_{4}\right](\mathrm{OTf})_{2}(\mathbf{1})$

S11 Figure S8. VT Mössbauer spectrum of $\left[\mathrm{Fe}_{3}\left(\mu_{2}-\mathrm{S}^{M e} \mathrm{NS}^{-}\right)_{4}\right](\mathrm{OTf})_{2}(\mathbf{1})$

S12 Figure S9. Variable temperature magnetic moment of $\left[\mathrm{Fe}_{3}\left(\mu_{2}-\mathrm{S}^{M e} \mathrm{NS}^{-}\right)_{4}\right](\mathrm{OTf})_{2}(\mathbf{1})$

S13 Figure S10. Temperature dependence of the $\chi T$ product at $0.1 \mathrm{~T}$ of $\left[\mathrm{Fe}_{3}\left(\mu_{2}-\mathrm{S}^{M e} \mathrm{NS}^{-}\right)_{4}\right](\mathrm{OTf})_{2}(\mathbf{1})$

S14 Figure S11. ${ }^{1} \mathrm{H}$ NMR spectrum $\left(300 \mathrm{MHz}, \mathrm{CD}_{3} \mathrm{CN}\right)$ of $\left\{\left[\mathrm{Fe}\left(\mu-\mathrm{S}^{M e} \mathrm{NS}^{-}\right)\left(\mathrm{PMePh}_{2}\right)\left(\mathrm{CH}_{3} \mathrm{CN}\right)\right]_{2}\right\}(\mathrm{OTf})_{2}(\mathbf{2})$

S15 Figure S12. ${ }^{31} \mathrm{P}\left\{{ }^{1} \mathrm{H}\right\} \mathrm{NMR}$ spectrum $\left(121 \mathrm{MHz}, \mathrm{CD}_{3} \mathrm{CN}\right)$ of $\left\{\left[\mathrm{Fe}\left(\mu-\mathrm{S}^{M e} \mathrm{NS}^{-}\right)\left(\mathrm{PMePh}_{2}\right)\left(\mathrm{CH}_{3} \mathrm{CN}\right)\right]_{2}\right\}(\mathrm{OTf})_{2}(\mathbf{2})$

S16 Figure S13. ${ }^{19} \mathrm{~F}$ NMR spectrum $\left(282 \mathrm{MHz}, \mathrm{CD}_{3} \mathrm{CN}\right)$ of $\left\{\left[\mathrm{Fe}\left(\mu-\mathrm{S}^{M e} \mathrm{NS}^{-}\right)\left(\mathrm{PMePh}_{2}\right)\left(\mathrm{CH}_{3} \mathrm{CN}\right)\right]_{2}\right\}(\mathrm{OTf})_{2}($ (2)

S17 Figure S14. ${ }^{1} \mathrm{H}$ NMR spectrum $\left(300 \mathrm{MHz}, \mathrm{CD}_{3} \mathrm{CN}\right)$ of $\left\{\left[\mathrm{Fe}\left(\mu-\mathrm{S}^{M e} \mathrm{NS}^{-}\right)(\mathrm{CNxylyl})_{2}\right]\right\}(\mathrm{OTf})_{2}(\mathbf{3})$ 
S18 Figure S15. ${ }^{13} \mathrm{C}\left\{{ }^{1} \mathrm{H}\right\}$ NMR spectrum $\left(75 \mathrm{MHz}, \mathrm{CD}_{3} \mathrm{CN}\right)$ of $\left\{\left[\mathrm{Fe}\left(\mu-\mathrm{S}^{M e} \mathrm{NS}^{-}\right)(\mathrm{CNxylyl})_{2}\right]\right\}(\mathrm{OTf})_{2}(3)$

S19 Figure S16. ${ }^{19} \mathrm{~F}$ NMR spectrum $\left(282 \mathrm{MHz}, \mathrm{CD}_{3} \mathrm{CN}\right)$ of $\left\{\left[\mathrm{Fe}\left(\mu-\mathrm{S}^{M e} \mathrm{NS}^{-}\right)(\mathrm{CNxylyl})_{2}\right]\right\}(\mathrm{OTf})_{2}(3)$

S20

Figure S17. IR spectrum of $\left\{\left[\mathrm{Fe}\left(\mu-\mathrm{S}^{M e} \mathrm{NS}^{-}\right)(\mathrm{CNxylyl})_{2}\right]\right\}(\mathrm{OTf})_{2}(\mathbf{3})$

S21

Figure S18. 5 K Mossbauer spectrum of $\left\{\left[\mathrm{Fe}\left(\mu-\mathrm{S}^{M e} \mathrm{NS}^{-}\right)\left(\mathrm{PMePh}_{2}\right)\left(\mathrm{CH}_{3} \mathrm{CN}\right)\right]_{2}\right\}(\mathrm{OTf})_{2}(\mathbf{2})$

S21

Figure S19. $80 \mathrm{~K}$ Mössbauer spectrum of $\left\{\left[\mathrm{Fe}\left(\mu-\mathrm{S}^{\mathrm{Me}} \mathrm{NS}^{-}\right)\left[\mathrm{P}(\mathrm{OMe})_{3}\right]_{2}\right]_{2}\right\}(\mathrm{OTf})_{2}(\mathbf{4})$

S22

Figure S20. ${ }^{1} \mathrm{H}$ NMR spectrum $\left(300 \mathrm{MHz}, \mathrm{CD}_{3} \mathrm{CN}\right)$ of $\left\{\left[\mathrm{Fe}\left(\mu-\mathrm{S}^{M e} \mathrm{NS}^{-}\right)\left[\mathrm{P}(\mathrm{OMe})_{3}\right]_{2}\right]_{2}\right\}(\mathrm{OTf})_{2}(4)$

S23

Figure S21. ${ }^{31} \mathrm{P}\left\{{ }^{1} \mathrm{H}\right\}$ NMR spectrum $\left(121 \mathrm{MHz}, \mathrm{CD}_{3} \mathrm{CN}\right)$ of $\left\{\left[\mathrm{Fe}\left(\mu-\mathrm{S}^{M e} \mathrm{NS}\right)\left[\mathrm{P}(\mathrm{OMe})_{3}\right]_{2}\right]_{2}\right\}(\mathrm{OTf})_{2}(\mathbf{4})$

S24

Figure S22. ${ }^{19} \mathrm{~F}$ NMR spectrum $\left(282 \mathrm{MHz}, \mathrm{CD}_{3} \mathrm{CN}\right)$ of $\left\{\left[\mathrm{Fe}\left(\mu-\mathrm{S}^{M e} \mathrm{NS}^{-}\right)\left[\mathrm{P}(\mathrm{OMe})_{3}\right]_{2}\right]_{2}\right\}(\mathrm{OTf})_{2}(4)$

S25

Figure S23. ORTEP diagram of $\left\{\left[\mathrm{Fe}\left(\mu-\mathrm{S}^{M e} \mathrm{NS}^{-}\right)\left(\mathrm{CH}_{3} \mathrm{CN}\right)\left[\mathrm{P}(\mathrm{OMe})_{3}\right]\right]_{2}\right\}(\mathrm{OTf})_{2}(\mathbf{4 a})$

S26

Figure S24. ${ }^{1} \mathrm{H}$ NMR spectrum $\left(300 \mathrm{MHz}, \mathrm{CD}_{3} \mathrm{CN}\right)$ of $\left\{\left[\mathrm{Fe}\left(\mu-\mathrm{S}^{M e} \mathrm{NS}^{-}\right)\left(\mathrm{CH}_{3} \mathrm{CN}\right)\left[\mathrm{P}(\mathrm{OMe})_{3}\right]\right]_{2}\right\}(\mathrm{OTf})_{2}(\mathbf{4 a})$

S27

Figure S25. ${ }^{31} \mathrm{P}\left\{{ }^{1} \mathrm{H}\right\}$ NMR spectrum $\left(121 \mathrm{MHz}, \mathrm{CD}_{3} \mathrm{CN}\right)$ of $\left\{\left[\mathrm{Fe}\left(\mu-\mathrm{S}^{M e} \mathrm{NS}\right)\left(\mathrm{CH}_{3} \mathrm{CN}\right)\left[\mathrm{P}(\mathrm{OMe})_{3}\right]\right]_{2}\right\}(\mathrm{OTf})_{2}$ (4a)

S28 Figure S26. ${ }^{1} \mathrm{H}$ NMR spectrum $\left(300 \mathrm{MHz}, \mathrm{CD}_{3} \mathrm{CN}\right)$ of the reaction of $\left[\mathrm{Fe}_{3}\left(\mu_{2}-\mathrm{S}^{M e} \mathrm{NS}^{-}\right)_{4}\right](\mathrm{OTf})_{2}(\mathbf{1})$ and $\mathrm{NaOTf}$ with excess $\mathrm{P}(\mathrm{OMe})_{3}$

Figure S27. ${ }^{31} \mathrm{P}\left\{{ }^{1} \mathrm{H}\right\}$ NMR spectrum $\left(121 \mathrm{MHz}, \mathrm{CD}_{3} \mathrm{CN}\right)$ of the reaction of $\left[\mathrm{Fe}_{3}\left(\mu_{2}-\mathrm{S}^{M e} \mathrm{NS}^{-}\right)_{4}\right](\mathrm{OTf})_{2}(\mathbf{1})$ and NaOTf with excess $\mathrm{P}(\mathrm{OMe})_{3}$

S30

Figure S28. ${ }^{1} \mathrm{H}$ NMR spectrum $\left(300 \mathrm{MHz}, \mathrm{CDCl}_{3}\right)$ of $\left[\mathrm{Fe}\left(\mathrm{S}^{\mathrm{Me}} \mathrm{NS}{ }^{-}\right)\left\{\mathrm{P}(\mathrm{OMe})_{3}\right\}_{3}\right](\mathrm{OTf})(\mathbf{5})$

S31

Figure S29. ${ }^{31} \mathrm{P}\left\{{ }^{1} \mathrm{H}\right\}$ NMR spectrum $\left(121 \mathrm{MHz}, \mathrm{CDCl}_{3}\right)$ of $\left[\mathrm{Fe}\left(\mathrm{S}^{M e} \mathrm{NS}^{-}\right)\left\{\mathrm{P}(\mathrm{OMe})_{3}\right\}_{3}\right](\mathrm{OTf})(\mathbf{5})$

S32

Figure S30. ${ }^{19} \mathrm{~F}$ NMR spectrum $\left(282 \mathrm{MHz}, \mathrm{CDCl}_{3}\right)$ of $\left[\mathrm{Fe}\left(\mathrm{S}^{\mathrm{Me}} \mathrm{NS}-\left\{\mathrm{P}(\mathrm{OMe})_{3}\right\}_{3}\right](\mathrm{OTf})(\mathbf{5})\right.$

S33

Figure S31. ESI-MS spectrum of $\left[\mathrm{Fe}\left(\mathrm{S}^{M e} \mathrm{NS}^{-}\right)\left\{\mathrm{P}(\mathrm{OMe})_{3}\right\}_{3}\right](\mathrm{OTf})(\mathbf{5})$

S34

Table S1. Energies and MPA-derived compositions of the frontier molecular orbitals of $\left[\mathrm{Fe}\left(\mathrm{S}^{M e} \mathrm{NS}^{-}\right)\left\{\mathrm{P}(\mathrm{OMe})_{3}\right\}_{3}\right](\mathrm{OTf})(\mathbf{5})$

S35 Table S2. TD-DFT-calculated electronic transitions of $\left[\mathrm{Fe}\left(\mathrm{S}^{M e} \mathrm{NS}^{-}\right)\left\{\mathrm{P}(\mathrm{OMe})_{3}\right\}_{3}\right](\mathrm{OTf})(\mathbf{5})$

S38 Figure S32. The HOMO of $\left[\mathrm{Fe}\left(\mathrm{S}^{M e} \mathrm{NS}^{-}\right)\left\{\mathrm{P}(\mathrm{OMe})_{3}\right\}_{3}\right](\mathrm{OTf})(\mathbf{5})$

S39 Figure S33. The LUMO of $\left[\mathrm{Fe}\left(\mathrm{S}^{M e} \mathrm{NS}^{-}\right)\left\{\mathrm{P}(\mathrm{OMe})_{3}\right\}_{3}\right](\mathrm{OTf})(\mathbf{5})$

S40 Figure S34. The HOMO-2 of $\left[\mathrm{Fe}\left(\mathrm{S}^{M e} \mathrm{NS}^{-}\right)\left\{\mathrm{P}(\mathrm{OMe})_{3}\right\}_{3}\right](\mathrm{OTf})(5)$

S41 Figure S35. ${ }^{31} \mathrm{P}\left\{{ }^{1} \mathrm{H}\right\}$ NMR spectrum $\left(121 \mathrm{MHz}, \mathrm{CD}_{3} \mathrm{CN}\right)$ of the reaction of $\left[\mathrm{Fe}_{3}\left(\mu_{2}-\mathrm{S}^{M e} \mathrm{NS}^{-}\right)_{4}\right](\mathrm{OTf})_{2}(\mathbf{1})$ and 
NaOTf with excess $\mathrm{PMe}_{3}$

S42 Figure S36. ${ }^{31} \mathrm{P}\left\{{ }^{1} \mathrm{H}\right\}$ NMR spectrum $\left(121 \mathrm{MHz}, \mathrm{CDCl}_{3}\right)$ of the reaction of $\left[\mathrm{Fe}_{3}\left(\mu_{2}-\mathrm{S}^{M e} \mathrm{NS}^{-}\right)_{4}\right](\mathrm{OTf})_{2}(\mathbf{1})$ and $\mathrm{NaOTf}$ with excess $\mathrm{PMe}_{3}$

S43 Figure S37. ${ }^{31} \mathrm{P}\left\{{ }^{1} \mathrm{H}\right\}$ NMR spectrum $\left\{121 \mathrm{MHz},\left(\mathrm{CD}_{3}\right)_{2} \mathrm{CO}\right\}$ of the reaction of $\left[\mathrm{Fe}_{3}\left(\mu_{2}-\mathrm{S}^{M e} \mathrm{NS}^{-}\right)_{4}\right](\mathrm{OTf})_{2}(\mathbf{1})$ and $\mathrm{NaOTf}$ with excess $\mathrm{PMe}_{3}$ at $-20^{\circ} \mathrm{C}$

S44 Figure S38. Cyclic voltammogram of $\left[\mathrm{Fe}_{3}\left(\mu_{2}-\mathrm{S}^{M e} \mathrm{NS}^{-}\right)_{4}\right](\mathrm{OTf})_{2}(\mathbf{1})$ in $\mathrm{CH}_{2} \mathrm{Cl}_{2}$

S45 Figure S39. Cyclic voltammogram of $\left\{\left[\mathrm{Fe}\left(\mu-\mathrm{S}^{M e} \mathrm{NS}^{-}\right)\left(\mathrm{PMePh}_{2}\right)\left(\mathrm{CH}_{3} \mathrm{CN}\right)\right]_{2}\right\}(\mathrm{OTf})_{2}(2)$ in $\mathrm{CH}_{2} \mathrm{Cl}_{2}$

S46 Experimental section

S48 Cartesian coordinates for the optimized geometries of $\left[\mathrm{Fe}_{3}\left(\mu_{2}-\mathrm{S}^{M e} \mathrm{NS}{ }^{-}\right)_{4}\right](\mathrm{OTf})_{2}(\mathbf{1})$

S58 Cartesian coordinates for the optimized geometries of $\left[\mathrm{Fe}\left(\mathrm{S}^{M e} \mathrm{NS}{ }^{-}\right)\left\{\mathrm{P}(\mathrm{OMe})_{3}\right\}_{3}\right](\mathrm{OTf})(5)$

S62 $\quad$ References 

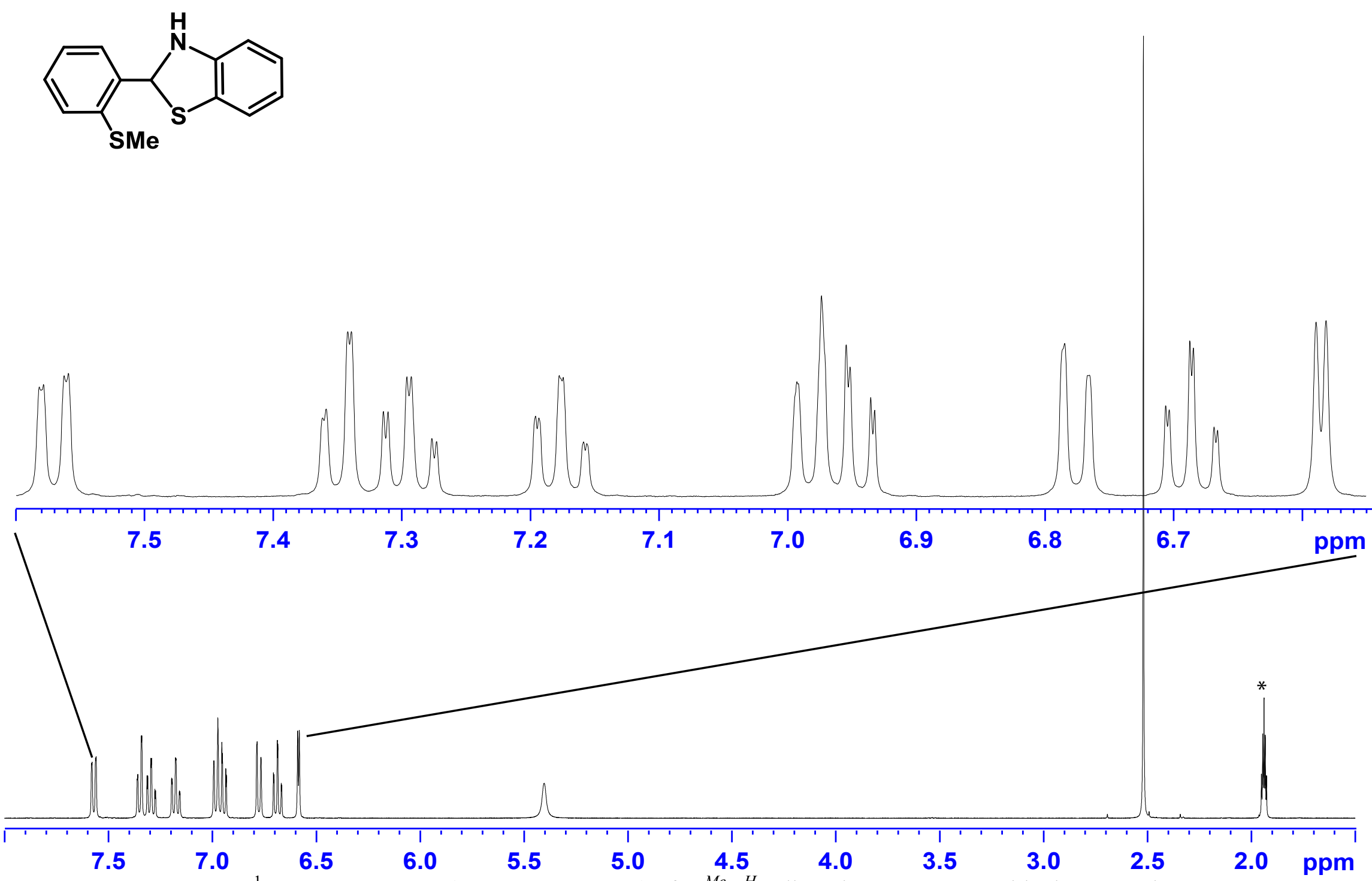

Figure S1. ${ }^{1} \mathrm{H}$ NMR spectrum $\left(300 \mathrm{MHz}, \mathrm{CD}_{3} \mathrm{CN}\right)$ of $\left[\mathrm{S}^{M e} \mathrm{~N}^{H} \mathrm{~S}\right.$ ] ligand precursor. [*residual NMR solvent]. 


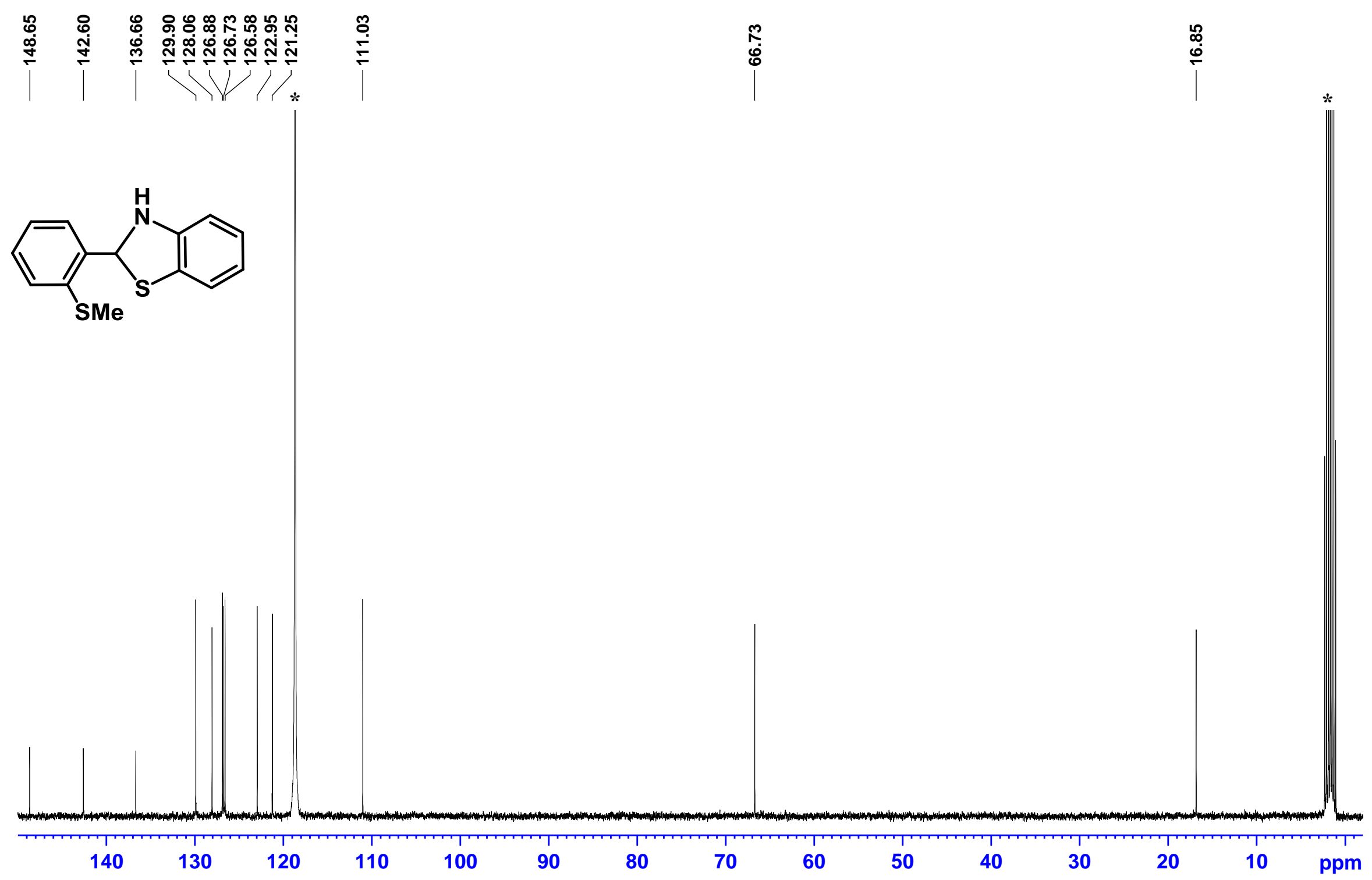

Figure S2. ${ }^{13} \mathrm{C}\left\{{ }^{1} \mathrm{H}\right\} \mathrm{NMR}$ spectrum $\left(101 \mathrm{MHz}, \mathrm{CD}_{3} \mathrm{CN}\right)$ of $\left[\mathrm{S}^{M e} \mathrm{~N}^{H} \mathrm{~S}\right]$ ligand precursor. [*residual NMR solvent]. 


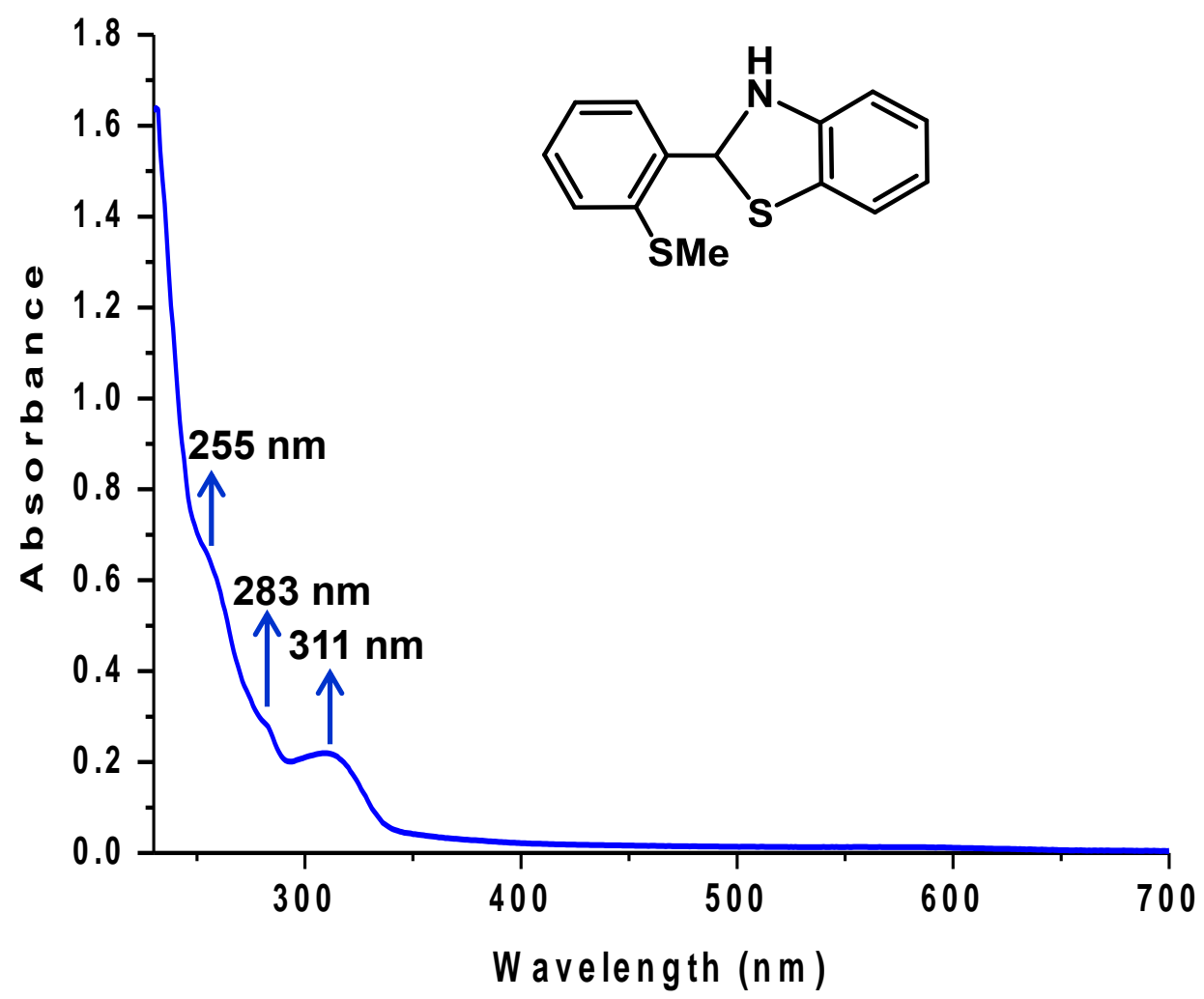

Figure S3. UV-vis spectrum of $\left[\mathrm{S}^{M e} \mathrm{~N}^{H} \mathrm{~S}\right]$ ligand precursor $\left(5 \times 10^{-3} \mathrm{M}\right.$ in dichloromethane $)$. 


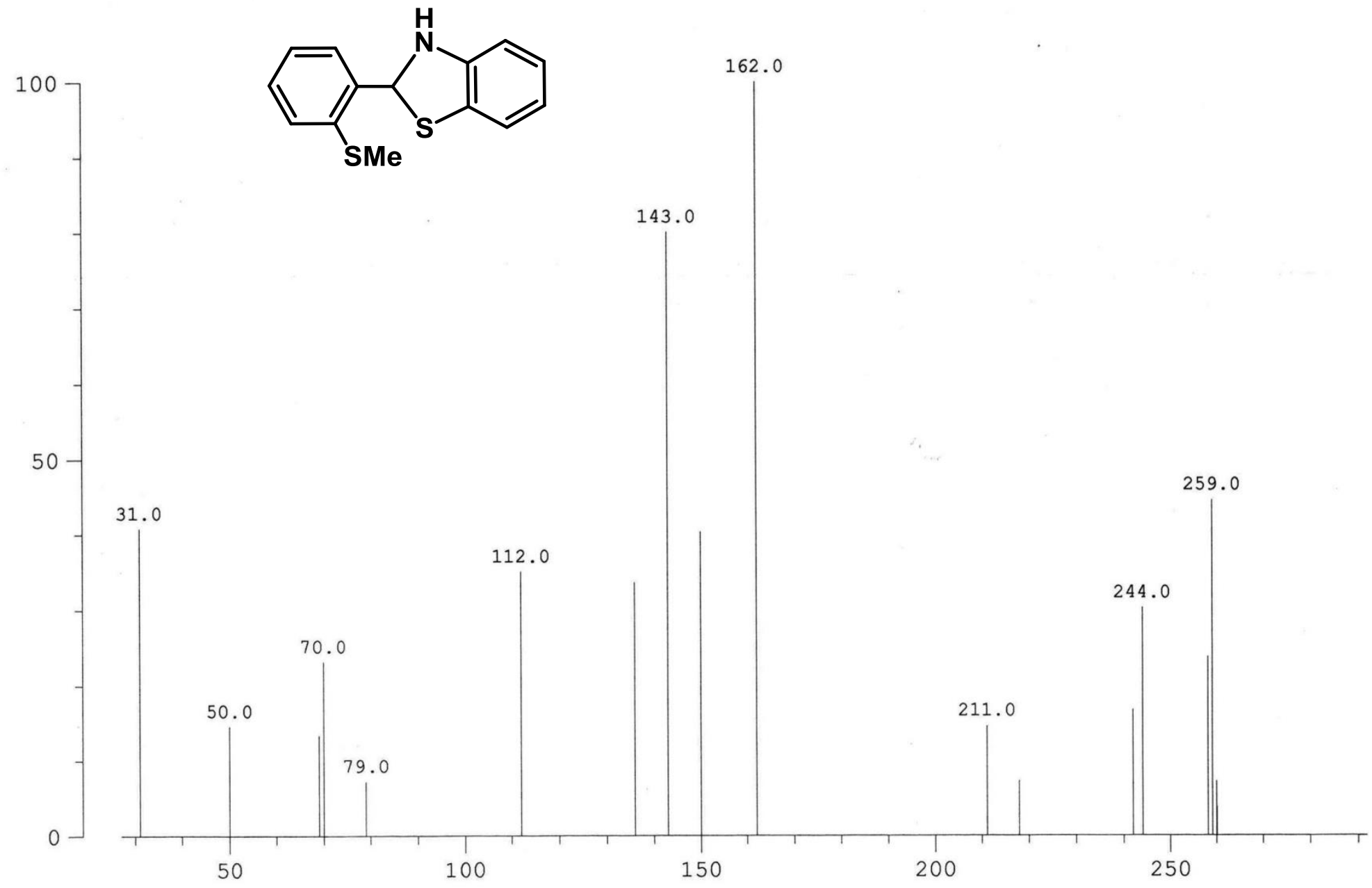

Figure S4. High-Resolution EI-MS spectrum of $\left[\mathrm{S}^{M e} \mathrm{~N}^{H} \mathrm{~S}\right]$ ligand precursor. 


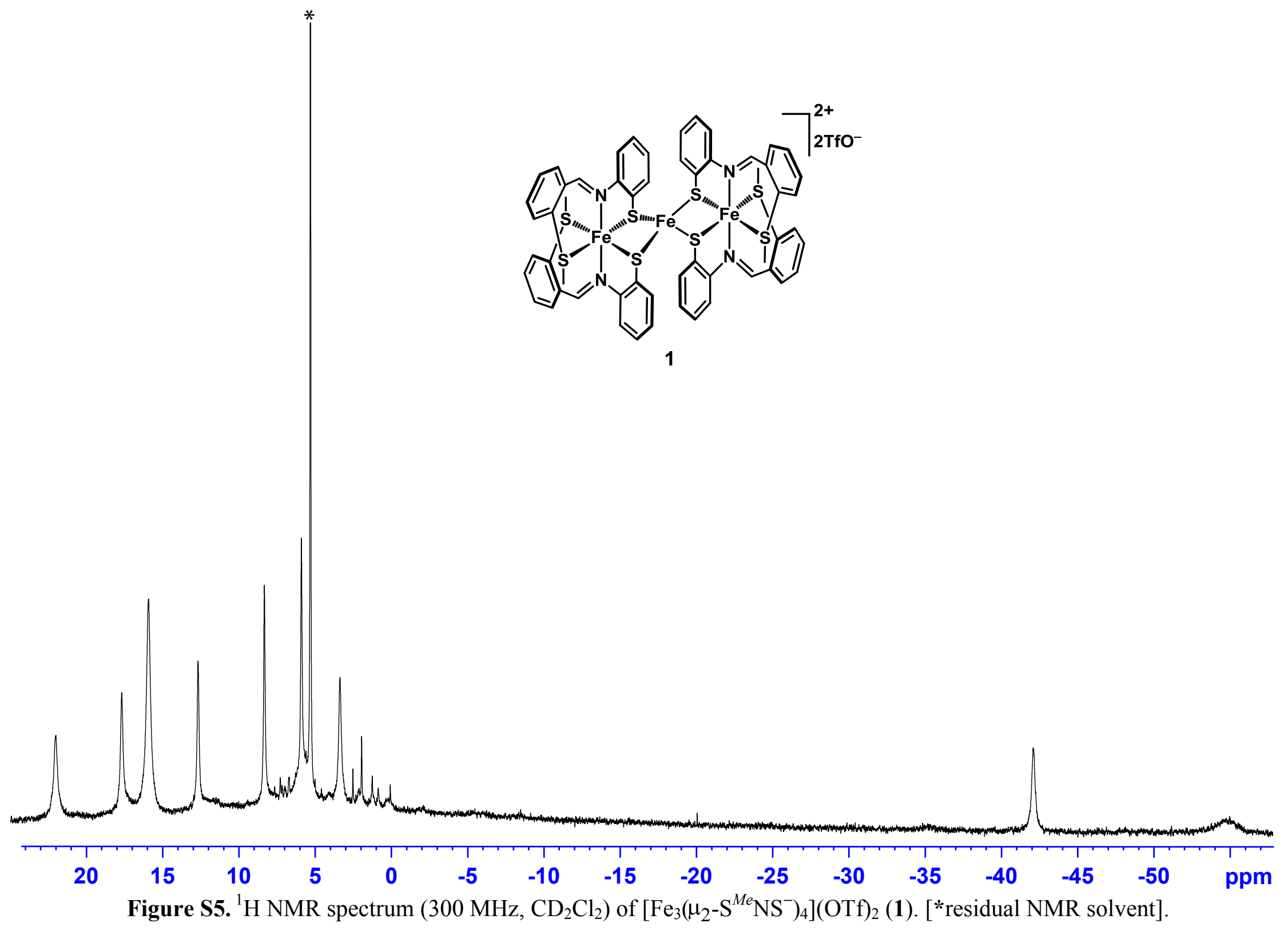




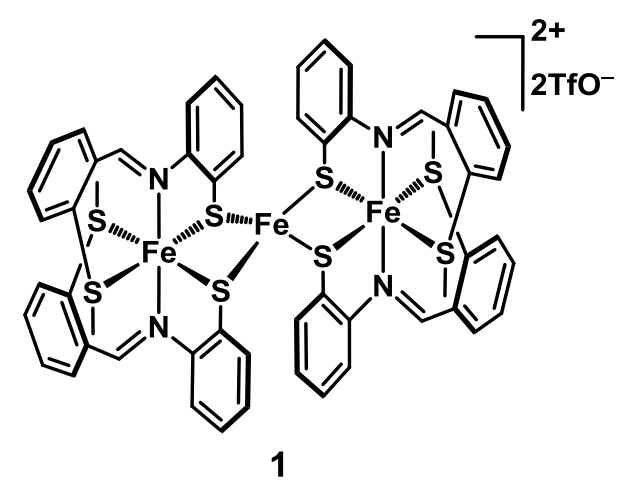

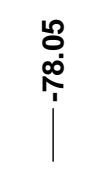

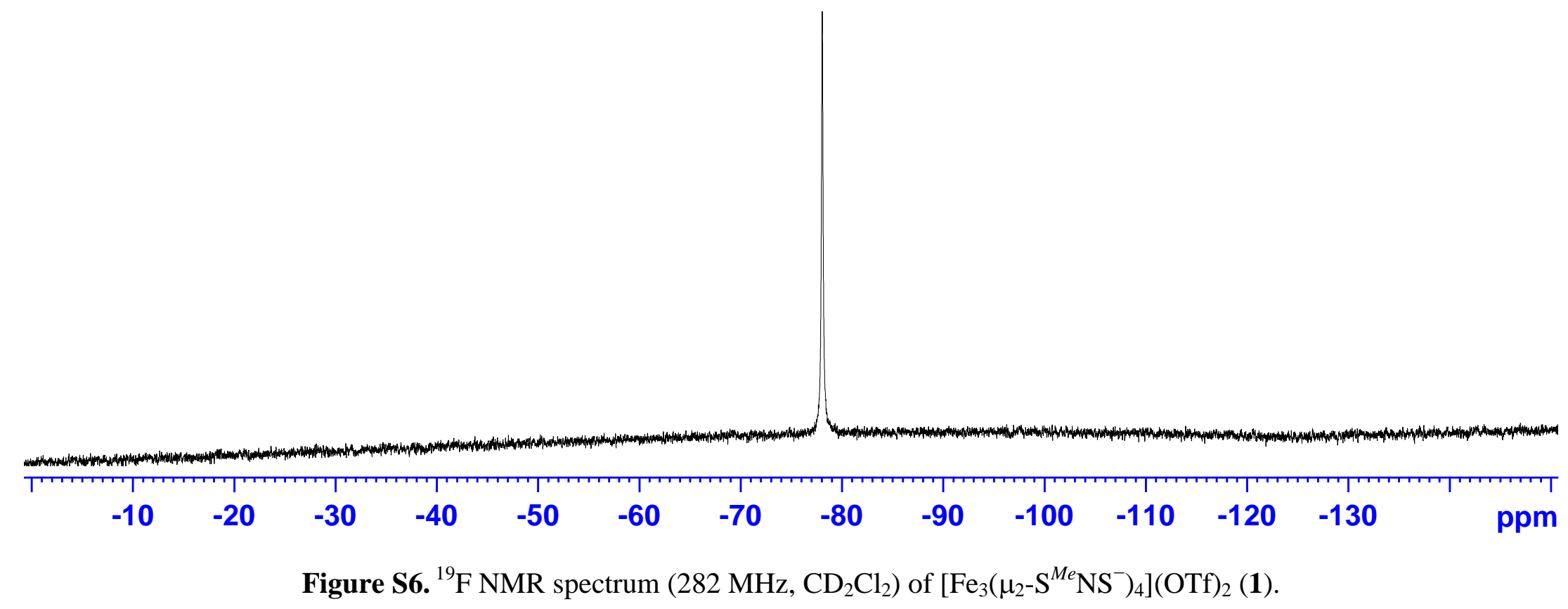




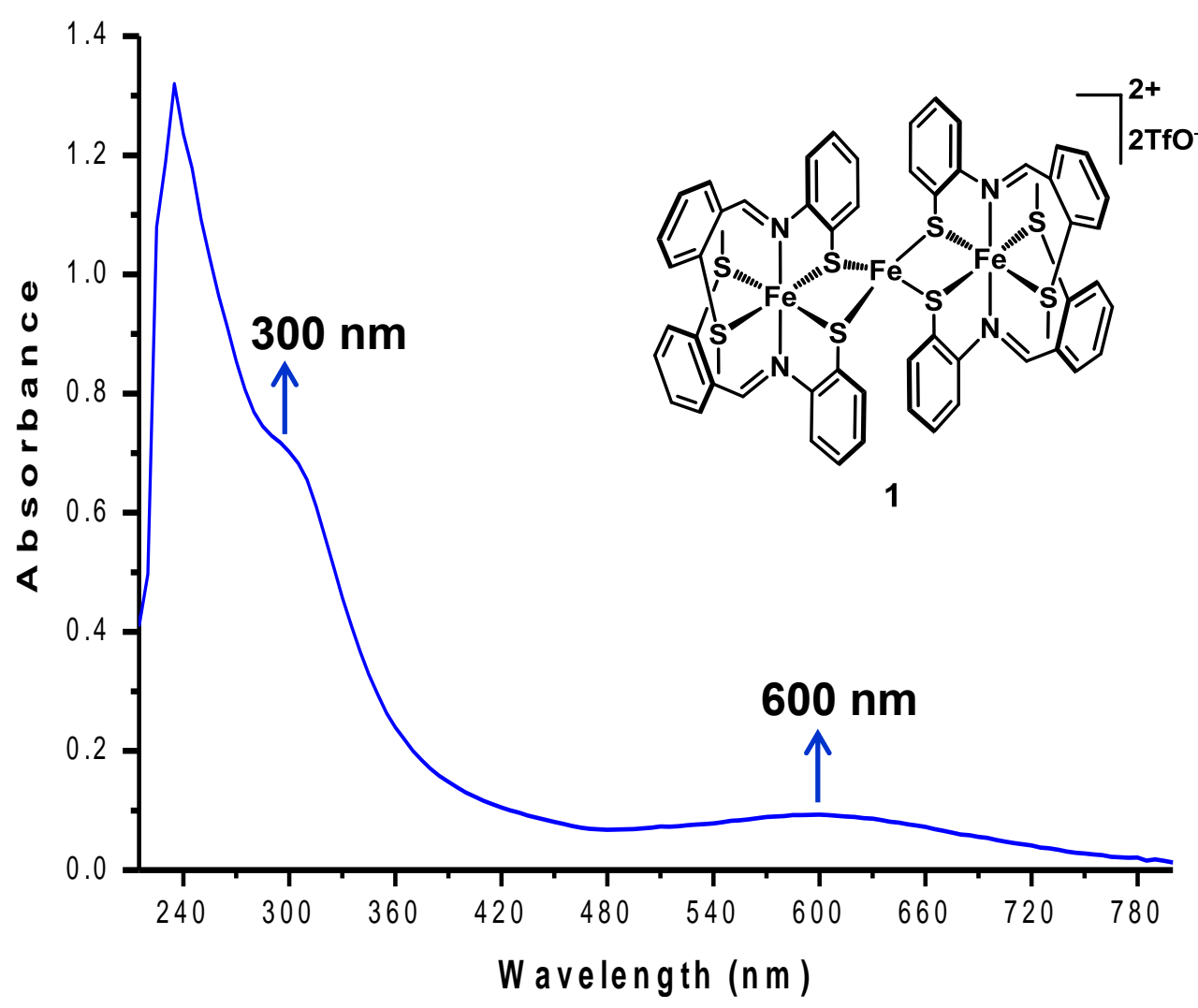

Figure S7. UV-vis spectrum of $\left[\mathrm{Fe}_{3}\left(\mu_{2}-\mathrm{S}^{M e} \mathrm{NS}^{-}\right)_{4}\right](\mathrm{OTf})_{2}(\mathbf{1})\left(1.7 \times 10^{-5} \mathrm{M}\right.$ in dichloromethane). 

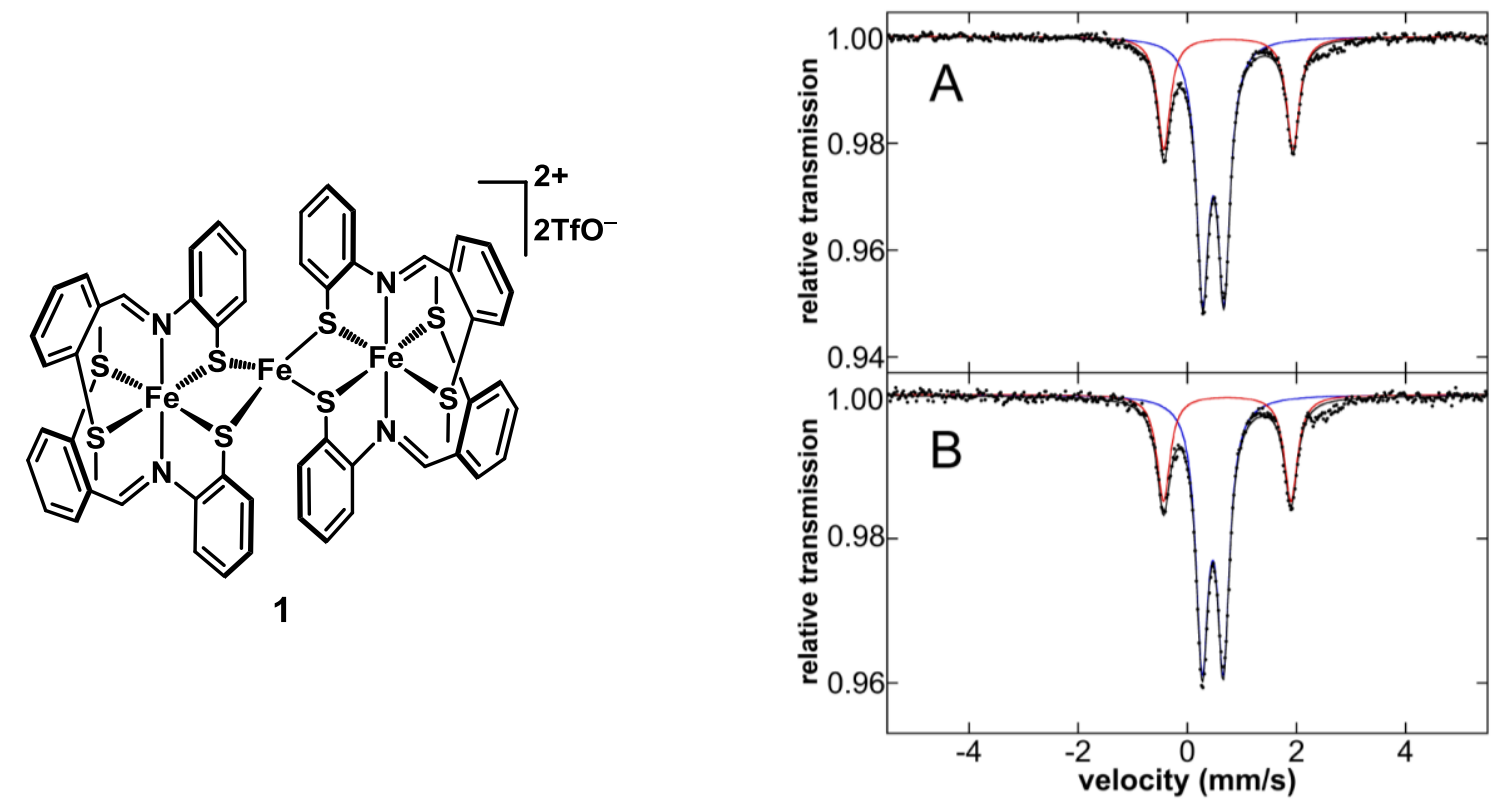

Figure S8. VT Mössbauer spectrum of $\left[\mathrm{Fe}_{3}\left(\mu_{2}-\mathrm{S}^{M e} \mathrm{NS}^{-}\right)_{4}\right](\mathrm{OTf})_{2}(\mathbf{1})$. (A) $80 \mathrm{~K}$ and (B) $130 \mathrm{~K}$ Mössbauer spectra. Data (black dots) and total fit (black lines) are shown. The Individual component parameters are the following. $\mathrm{T}=80 \mathrm{~K}: \delta=0.48, \Delta \mathrm{E}_{\mathrm{Q}}=0.39$ (blue, $65 \%)$ and $\delta=0.76, \Delta \mathrm{E}_{\mathrm{Q}}=2.37(\mathrm{red}, 35 \%) \mathrm{T}=130 \mathrm{~K}: \delta=0.47, \Delta \mathrm{E}_{\mathrm{Q}}=0.39\left(\right.$ blue, 69\%) and $\delta=0.76, \Delta \mathrm{E}_{\mathrm{Q}}=2.33(\mathrm{red}, 31 \%)$. 


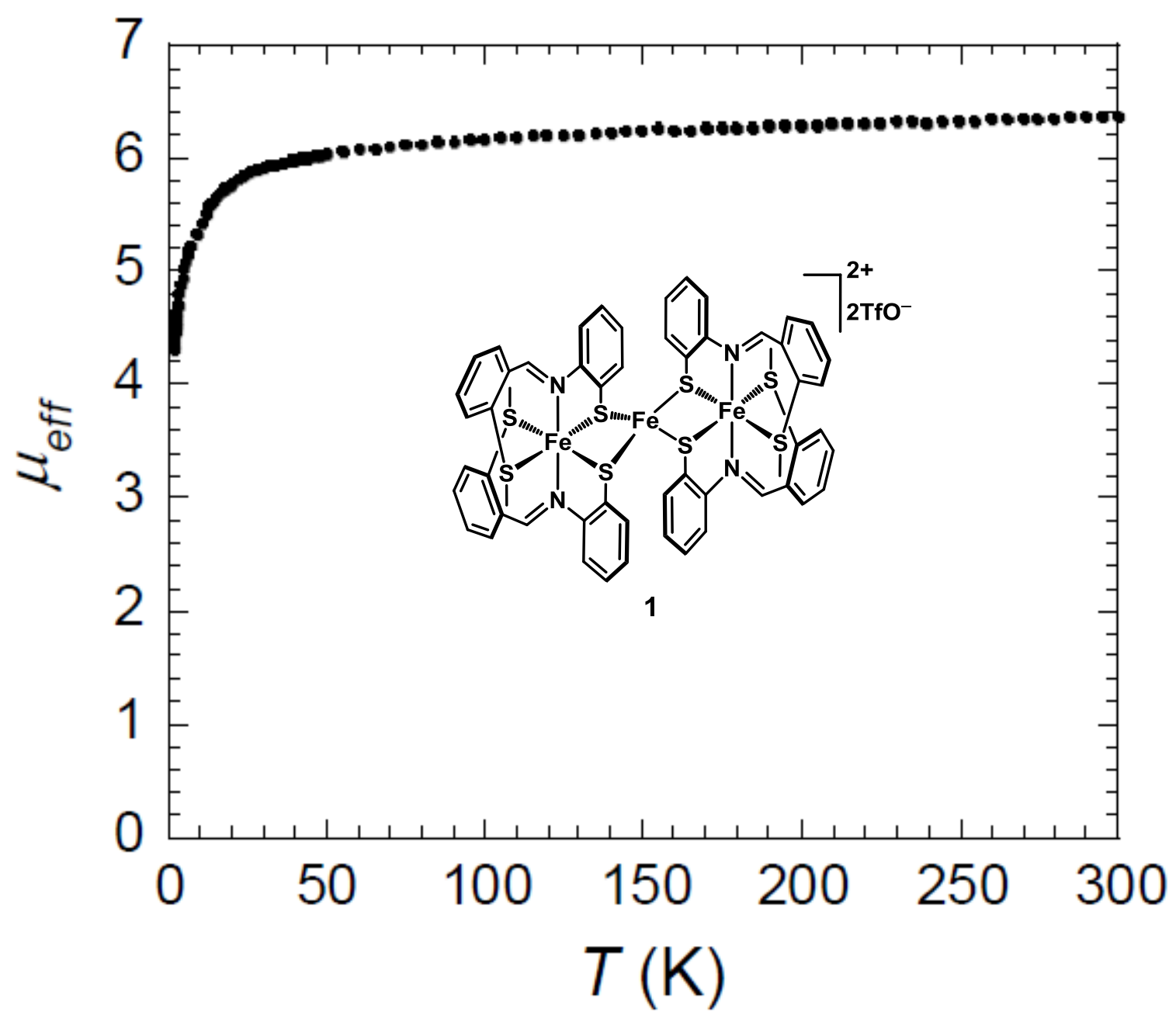

Figure S9. Variable temperature magnetic moment of $\left[\mathrm{Fe}_{3}\left(\mu_{2}-\mathrm{S}^{M e} \mathrm{NS}^{-}\right)_{4}\right](\mathrm{OTf})_{2}(\mathbf{1})$ under an applied dc field of $0.1 \mathrm{~T}$. 


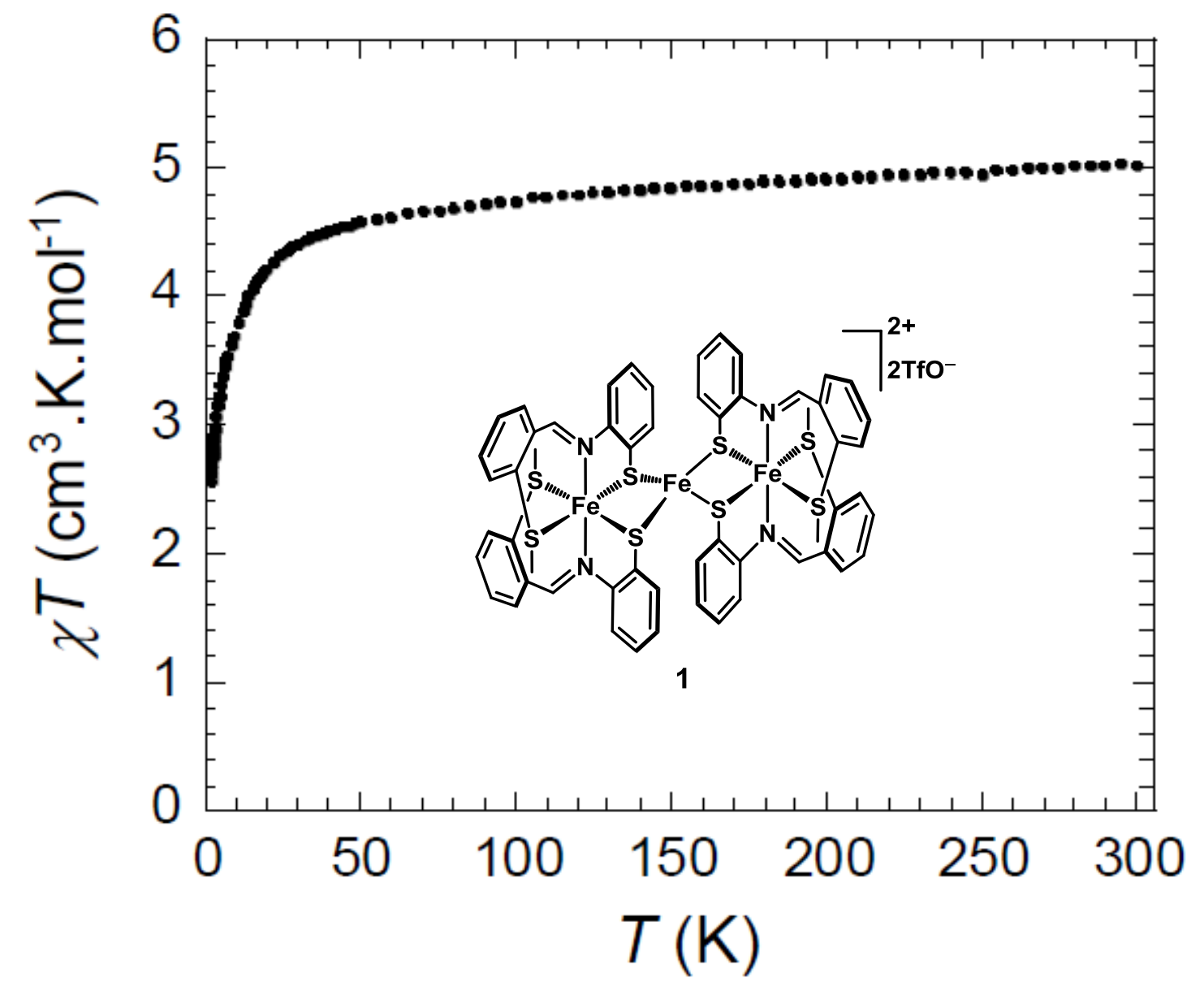

Figure S10. Temperature dependence of the $\chi T$ product at $0.1 \mathrm{~T}$ for complex $\left[\mathrm{Fe}_{3}\left(\mu_{2}-\mathrm{S}^{M e} \mathrm{NS}^{-}\right)_{4}\right](\mathrm{OTf})_{2}(\mathbf{1})$ (with $\chi$ being the molar susceptibility per molecule defined as $\mathrm{M} / \mathrm{H})$. 


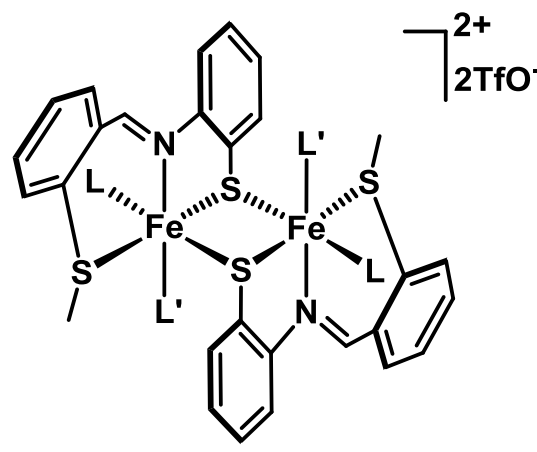

2, L=PMePh ${ }_{2}, \mathrm{~L}^{\prime}=\mathrm{CH}_{3} \mathrm{CN}$
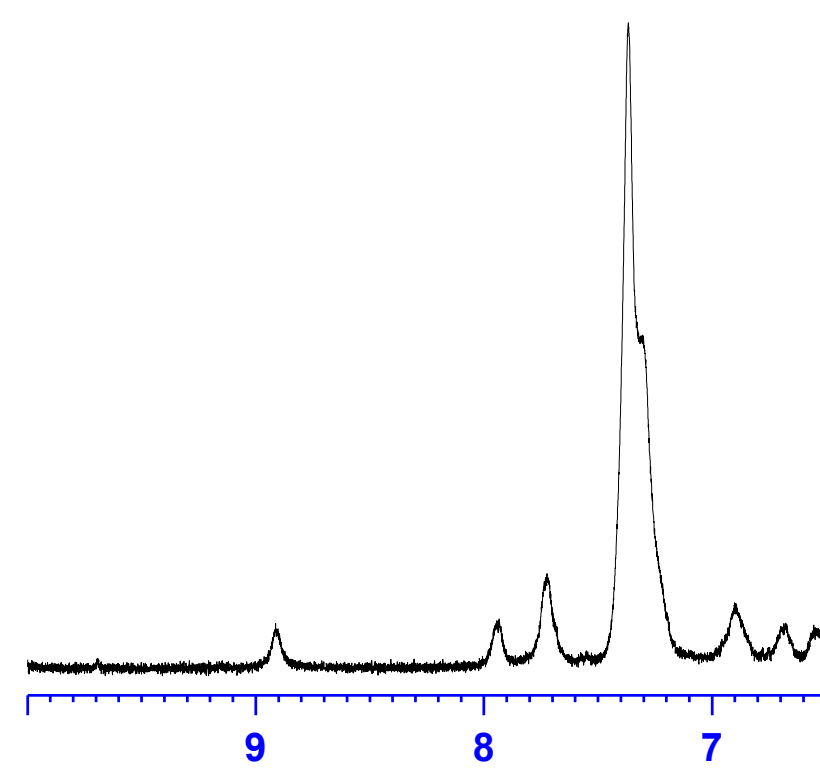

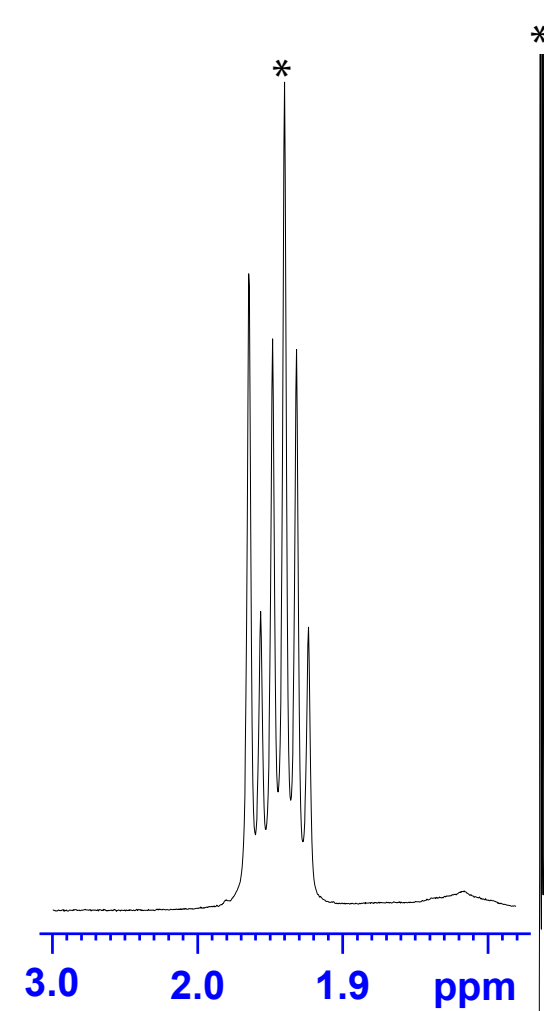

Figure S11. ${ }^{1} \mathrm{H}$ NMR spectrum $\left(300 \mathrm{MHz}, \mathrm{CD}_{3} \mathrm{CN}\right)$ of $\left\{\left[\mathrm{Fe}\left(\mu-\mathrm{S}^{M e} \mathrm{NS}^{-}\right)\left(\mathrm{PMePh}_{2}\right)\left(\mathrm{CH}_{3} \mathrm{CN}\right)\right]_{2}\right\}(\mathrm{OTf})_{2}(\mathbf{2})$. [ ${ }^{*} \mathrm{NMR}$ solvent; $\left.{ }^{\#} \mathrm{THF}\right]$ 

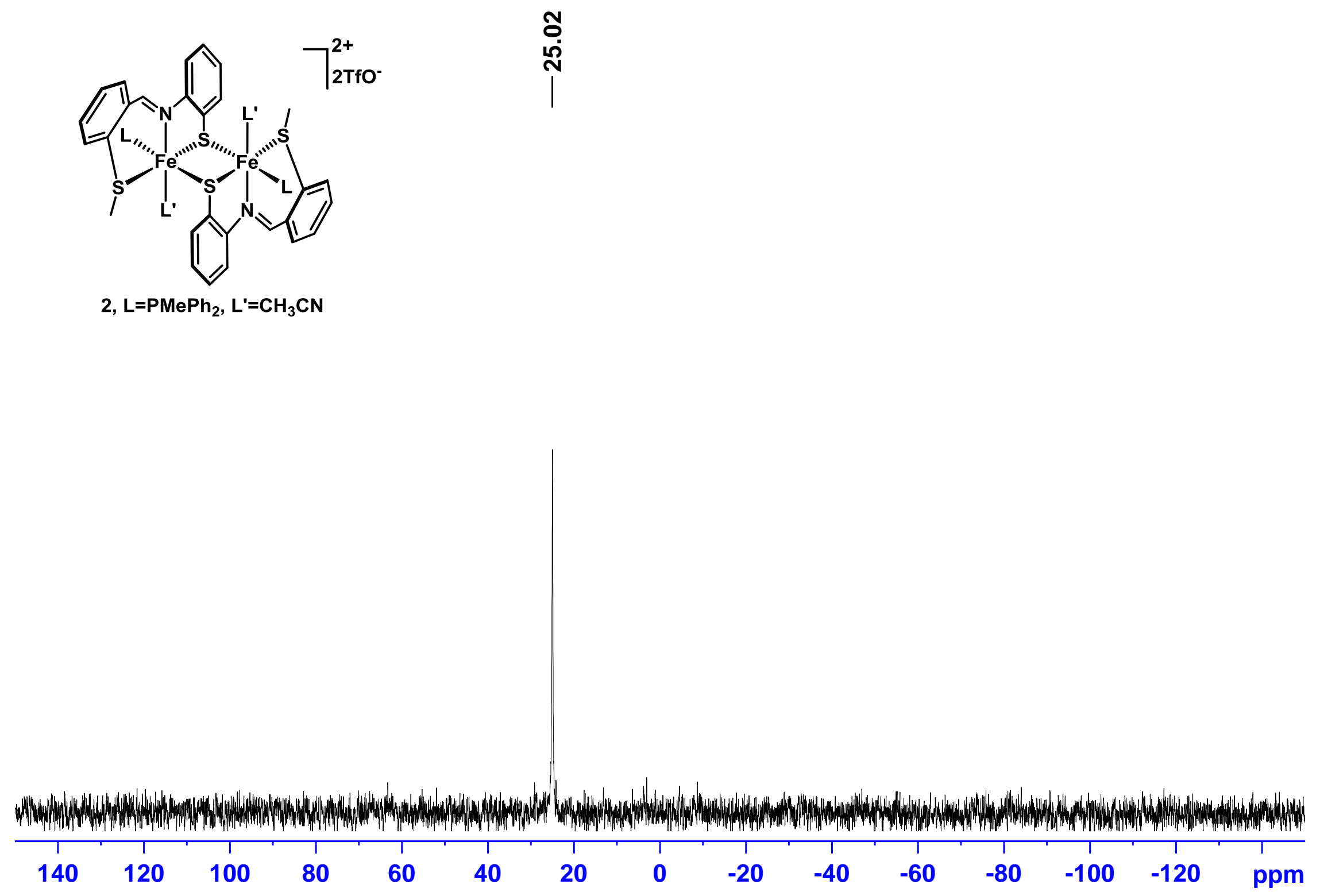

Figure S12. ${ }^{31} \mathrm{P}\left\{{ }^{1} \mathrm{H}\right\}$ NMR spectrum $\left(121 \mathrm{MHz}, \mathrm{CD}_{3} \mathrm{CN}\right)$ of $\left\{\left[\mathrm{Fe}\left(\mu-\mathrm{S}^{M e} \mathrm{NS}^{-}\right)\left(\mathrm{PMePh}_{2}\right)\left(\mathrm{CH}_{3} \mathrm{CN}\right)\right]_{2}\right\}(\mathrm{OTf})_{2}(2) .(\mathrm{LB}=3 \mathrm{~Hz})$. 


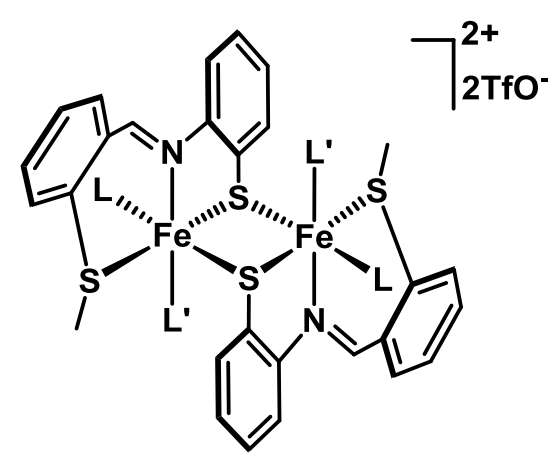

2, $\mathrm{L}=\mathrm{PMePh}_{2}, \mathrm{~L}^{\prime}=\mathrm{CH}_{3} \mathrm{CN}$

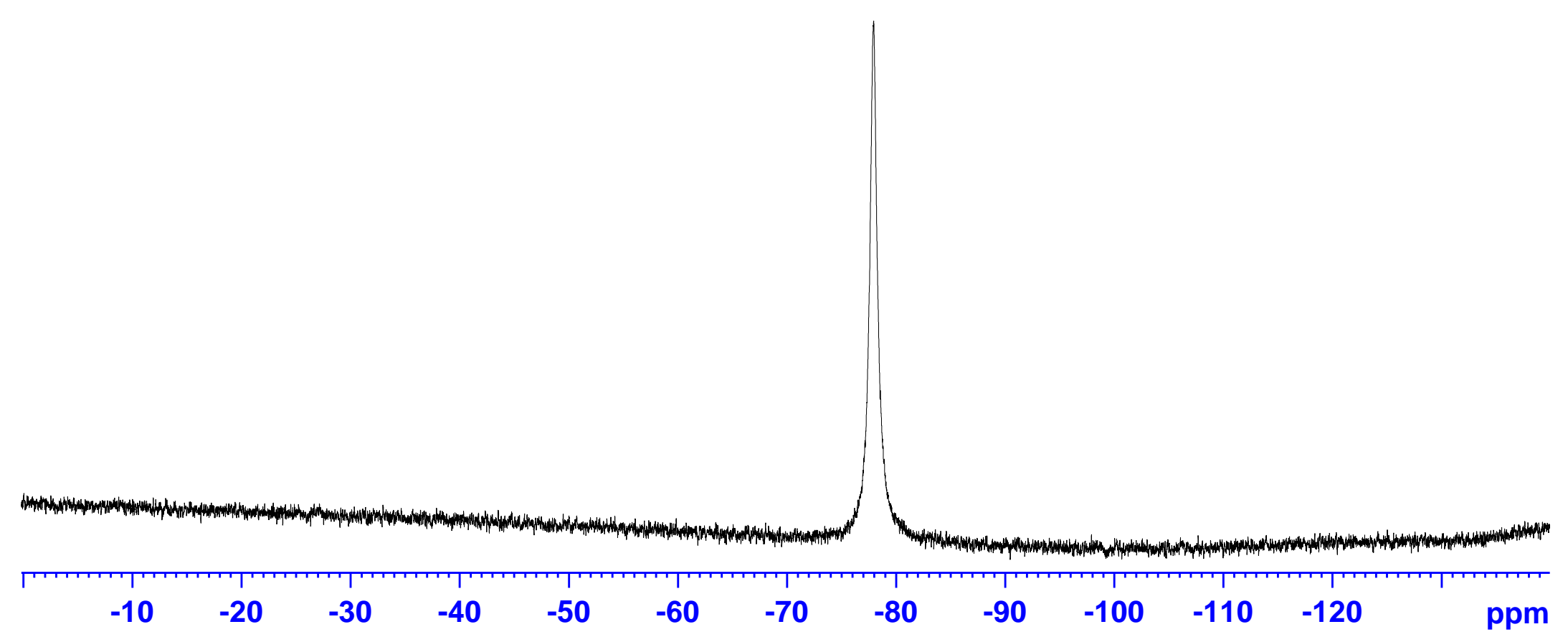

Figure S13. ${ }^{19} \mathrm{~F}$ NMR spectrum $\left(282 \mathrm{MHz}, \mathrm{CD}_{3} \mathrm{CN}\right)$ of $\left\{\left[\mathrm{Fe}\left(\mu-\mathrm{S}^{M e} \mathrm{NS}^{-}\right)\left(\mathrm{PMePh}_{2}\right)\left(\mathrm{CH}_{3} \mathrm{CN}\right)\right]_{2}\right\}(\mathrm{OTf})_{2}(\mathbf{2})$. 

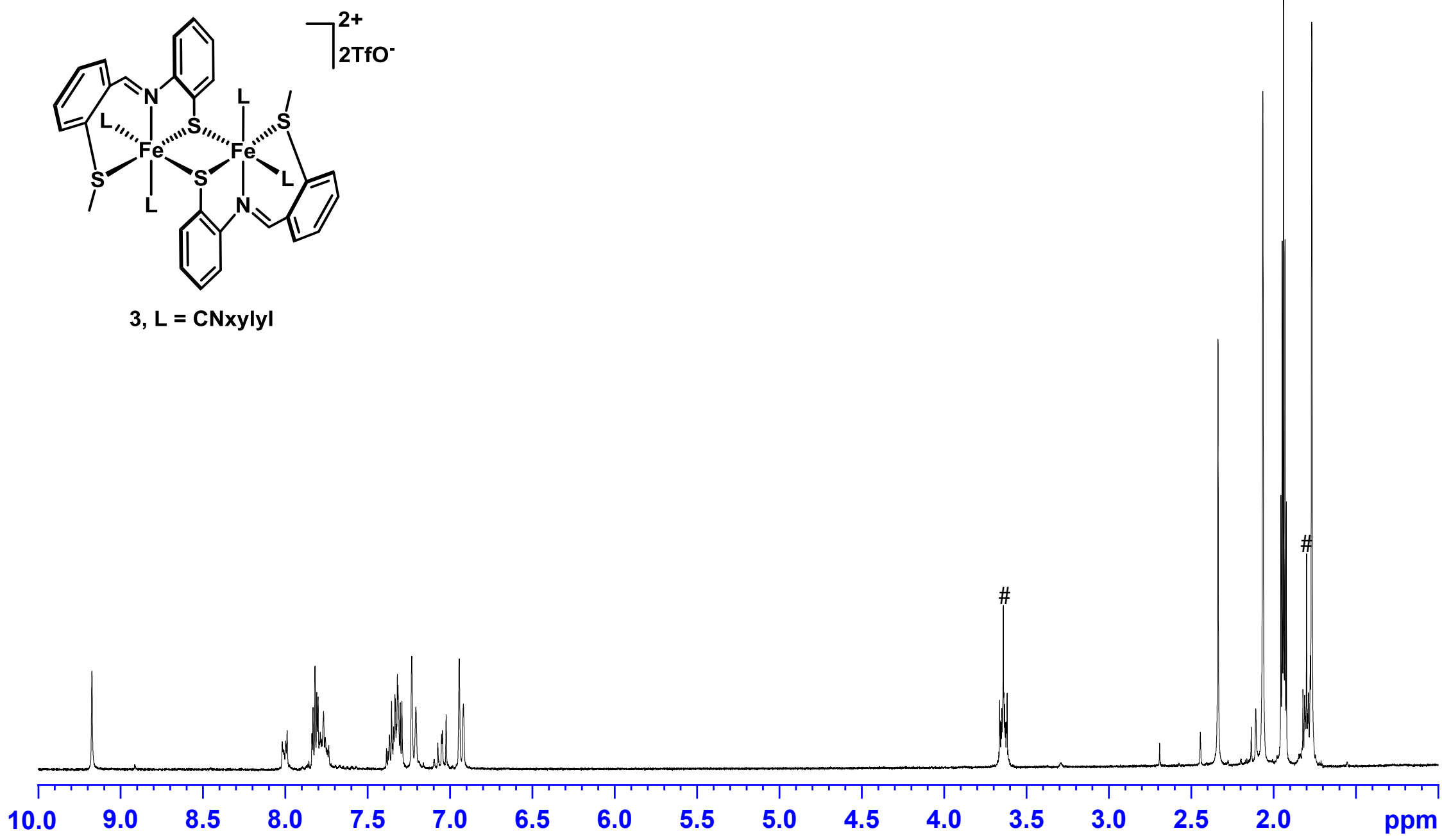

Figure S14. ${ }^{1} \mathrm{H}$ NMR spectrum $\left(300 \mathrm{MHz}, \mathrm{CD}_{3} \mathrm{CN}\right)$ of $\left\{\left[\mathrm{Fe}\left(\mu-\mathrm{S}^{\mathrm{Me}} \mathrm{NS}^{-}\right)(\mathrm{CNxylyl})_{2}\right]_{2}\right\}(\mathrm{OTf})_{2}(\mathbf{3})$. [ ${ }^{*} \mathrm{NMR}$ solvent; $\left.{ }^{\#} \mathrm{THF}\right]$ 


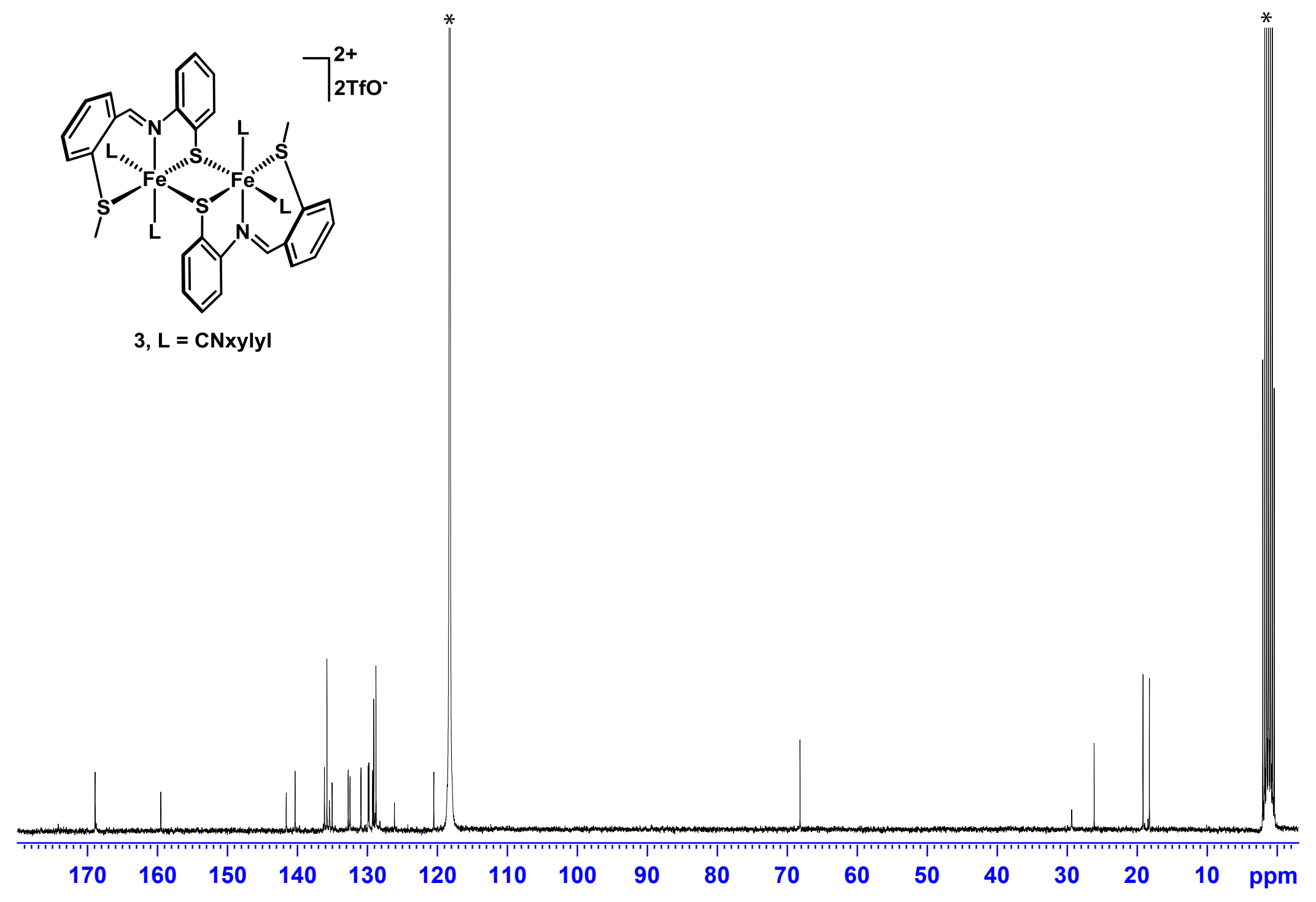

Figure S15. ${ }^{13} \mathrm{C}\left\{{ }^{1} \mathrm{H}\right\}$ NMR spectrum $\left(75 \mathrm{MHz}, \mathrm{CD}_{3} \mathrm{CN}\right)$ of $\left\{\left[\mathrm{Fe}\left(\mu-\mathrm{S}^{M e} \mathrm{NS}{ }^{-}\right)(\mathrm{CNxylyl})_{2}\right]_{2}\right\}(\mathrm{OTf})_{2}($ (3). [*residual NMR solvent] 

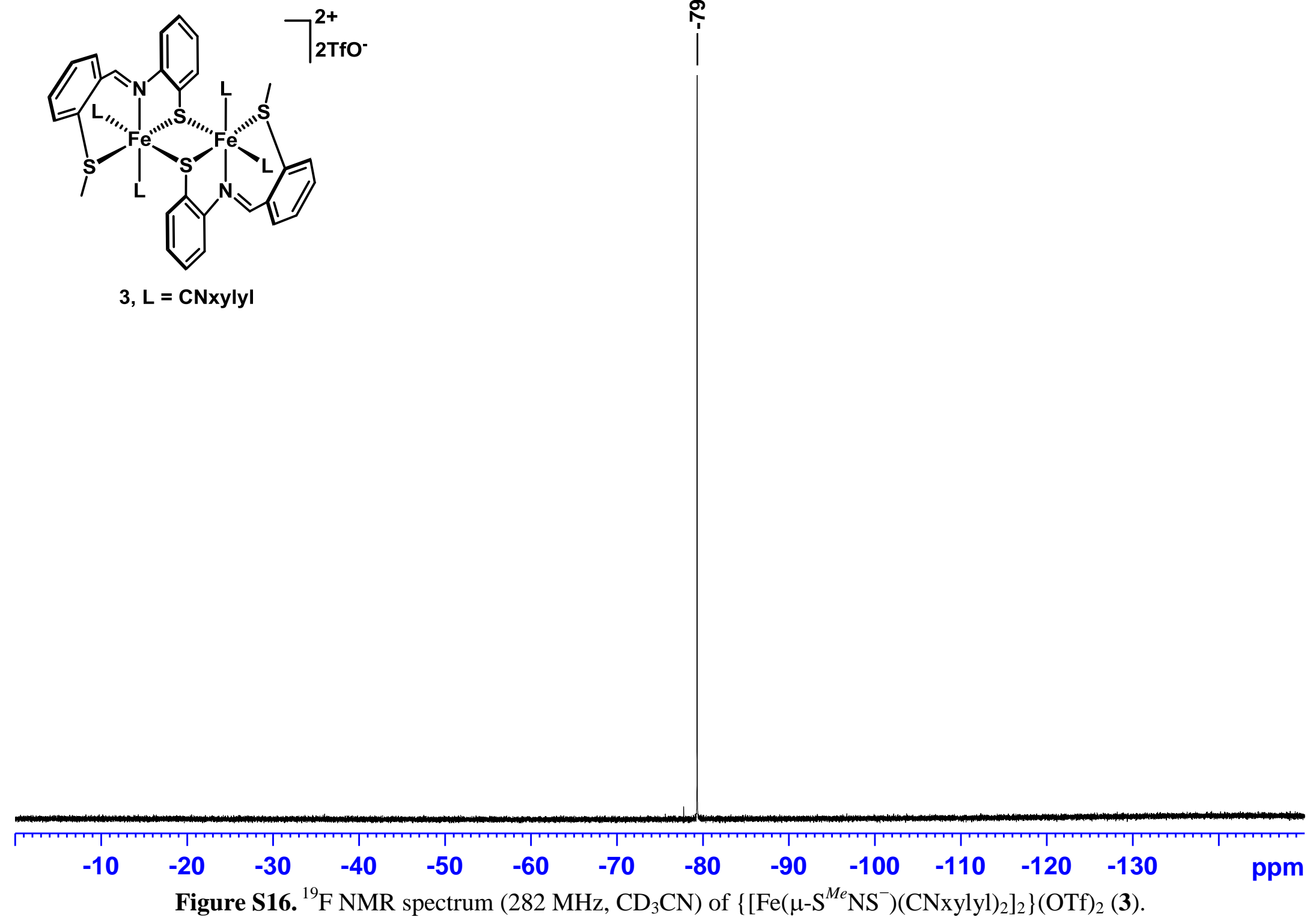


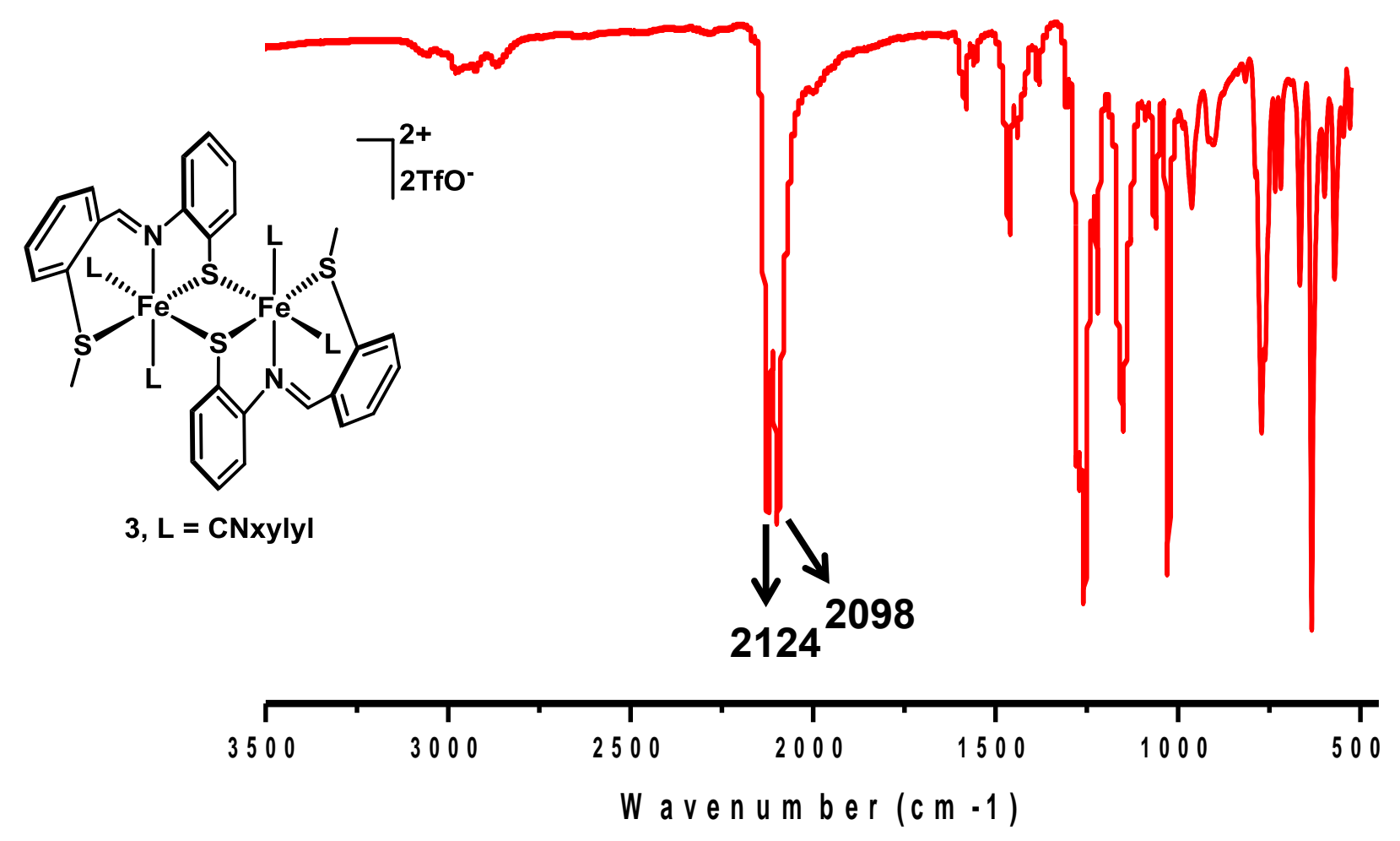

Figure S17. IR (ATR) spectrum of $\left\{\left[\mathrm{Fe}\left(\mu-\mathrm{S}^{M e} \mathrm{NS}^{-}\right)(\mathrm{CNxylyl})_{2}\right]_{2}\right\}(\mathrm{OTf})_{2}(3)$. 


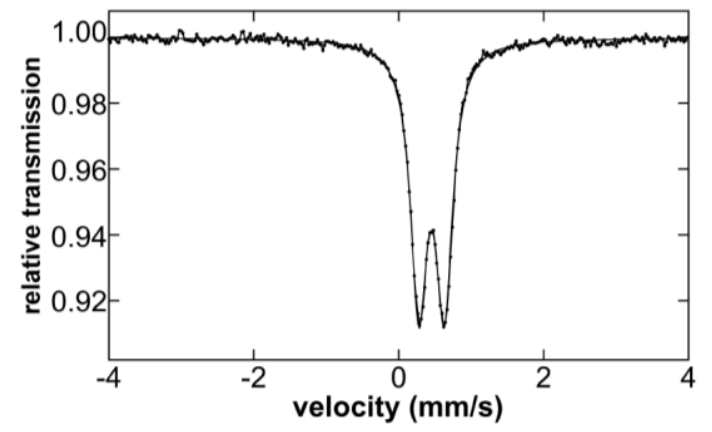

Figure S18. 5 K Mössbauer spectrum of $\left\{\left[\mathrm{Fe}\left(\mu-\mathrm{S}^{M e} \mathrm{NS}^{-}\right)\left(\mathrm{PMePh}_{2}\right)\left(\mathrm{CH}_{3} \mathrm{CN}\right)\right]_{2}\right\}(\mathrm{OTf})_{2}(2)$. The doublet is well-fit to the Mössbauer parameters $\delta=0.45$ and $\Delta \mathrm{E}_{\mathrm{Q}}=0.35$.

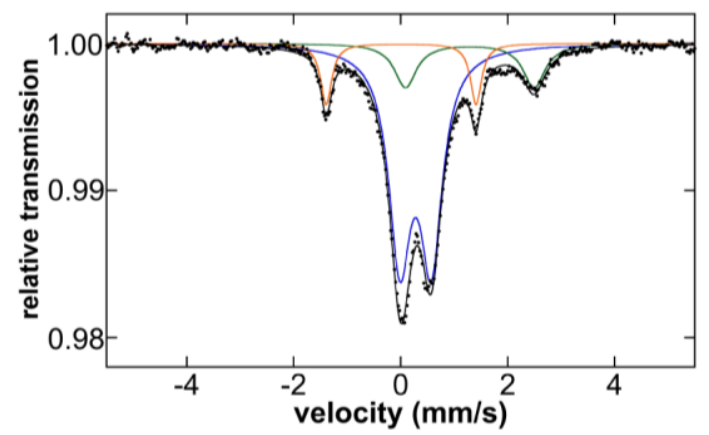

Figure S19. $80 \mathrm{~K}$ Mössbauer spectrum of $\left\{\left[\mathrm{Fe}_{2}\left(\mu-\mathrm{S}^{M e} \mathrm{NS}^{-}\right)\left[\mathrm{P}(\mathrm{OMe})_{3}\right]_{2}\right]_{2}\right\}(\mathrm{OTf})_{2}(4)$. The major species is fit to $\delta=0.28$ and $\Delta \mathrm{E}_{\mathrm{Q}}=$ 0.59 (blue, 72\%). Two additional components are observed with $\delta=1.29, \Delta \mathrm{E}_{\mathrm{Q}}=2.40$ (green, $16 \%$ ) and $\delta=0.01, \Delta \mathrm{E}_{\mathrm{Q}}=2.80($ orange, $11 \%)$. 

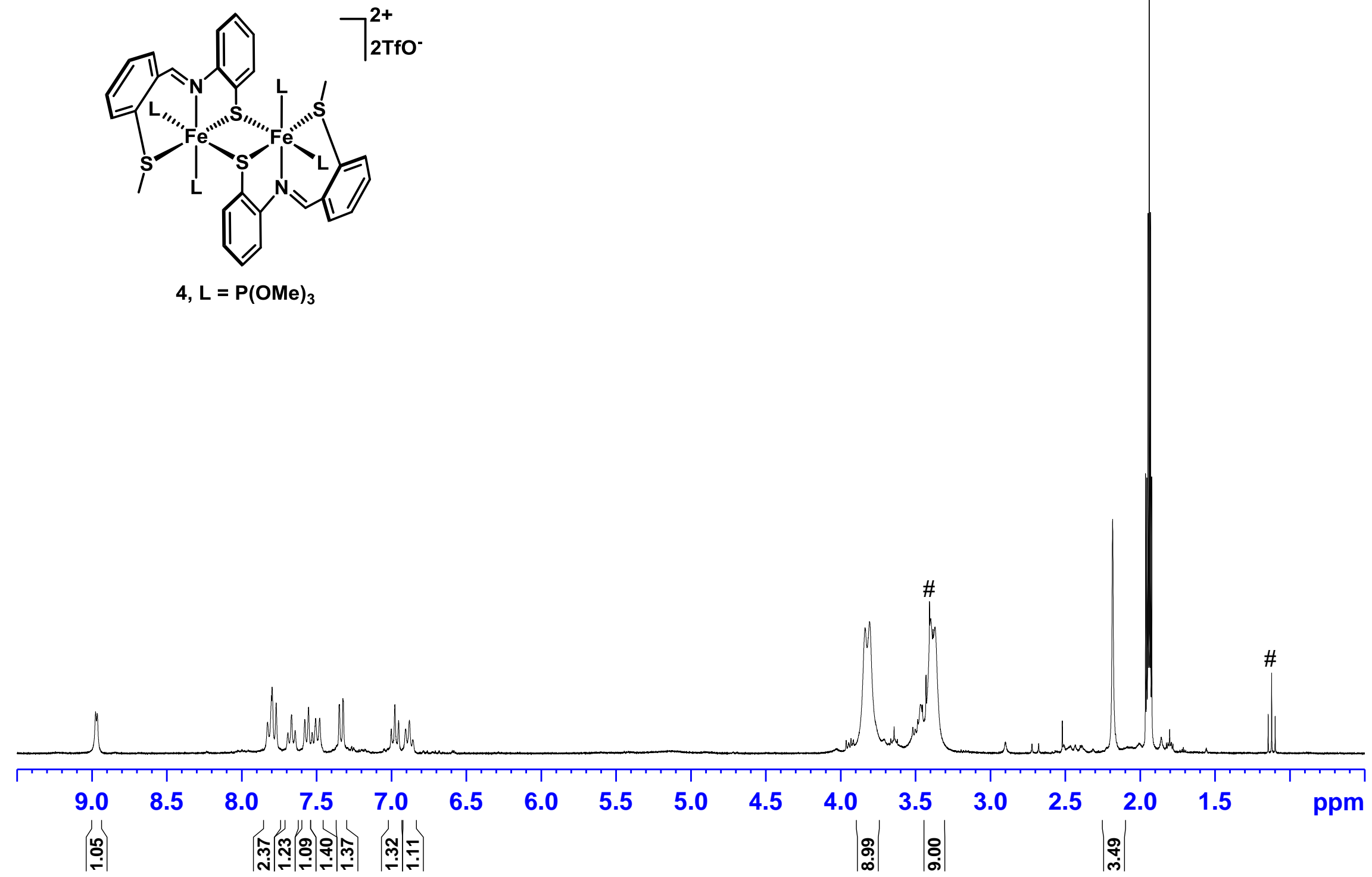

Figure S20. ${ }^{1} \mathrm{H}$ NMR spectrum $\left(300 \mathrm{MHz}, \mathrm{CD}_{3} \mathrm{CN}\right)$ of $\left\{\left[\mathrm{Fe}\left(\mu-\mathrm{S}^{M e} \mathrm{NS}^{-}\right)\left[\mathrm{P}(\mathrm{OMe})_{3}\right]_{2}\right]_{2}\right\}(\mathrm{OTf})_{2}(\mathbf{4})$. [*NMR solvent; ${ }^{*}$ diethyl ether] 

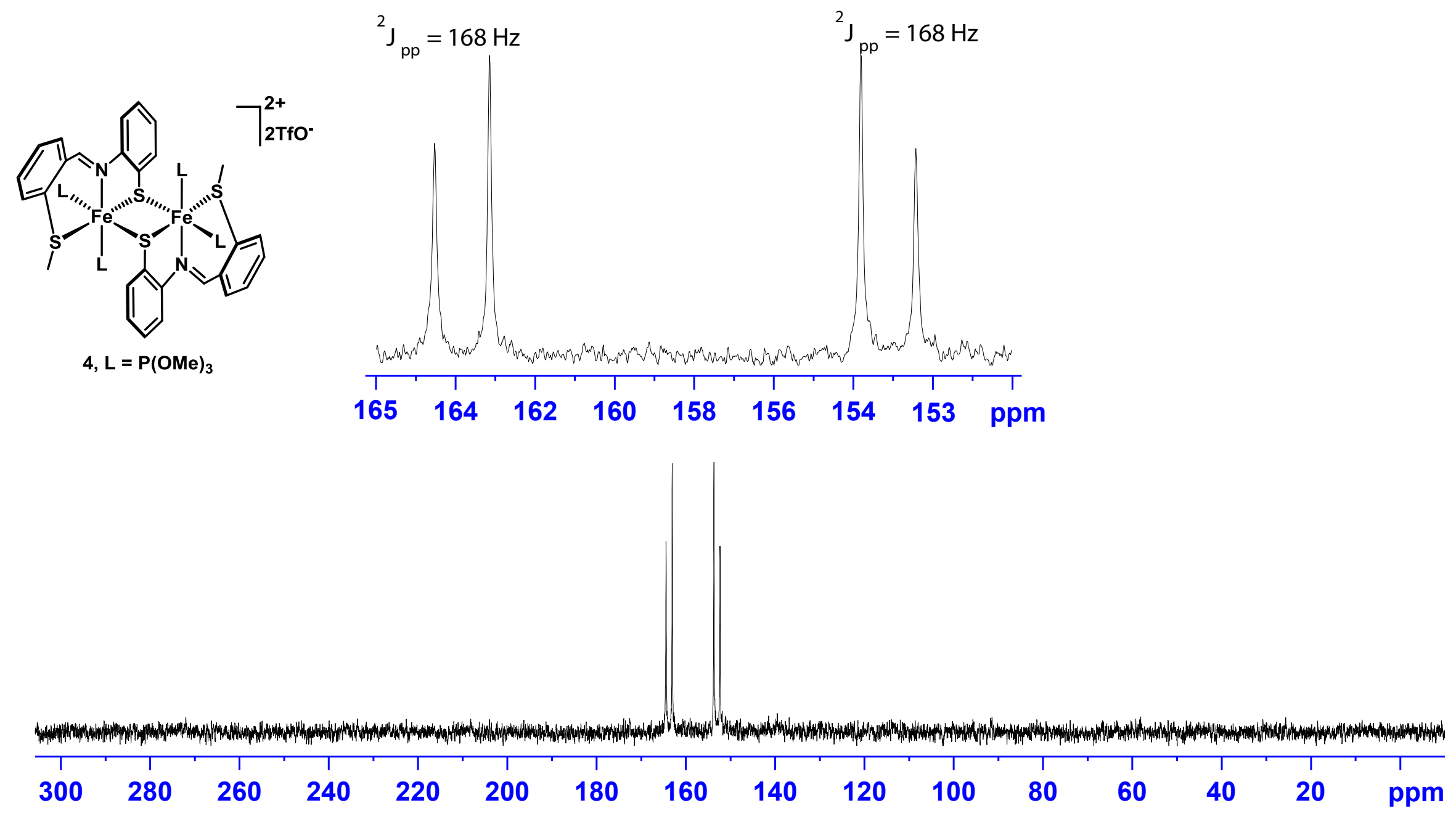

Figure S21. ${ }^{31} \mathrm{P}\left\{{ }^{1} \mathrm{H}\right\}$ NMR spectrum $\left(121 \mathrm{MHz}, \mathrm{CD}_{3} \mathrm{CN}\right)$ of $\left\{\left[\mathrm{Fe}\left(\mu-\mathrm{S}^{M e} \mathrm{NS}^{-}\right)\left[\mathrm{P}(\mathrm{OMe})_{3}\right]_{2}\right]_{2}\right\}(\mathrm{OTf})_{2}(\mathbf{4}) .(\mathrm{LB}=5 \mathrm{~Hz})$. 


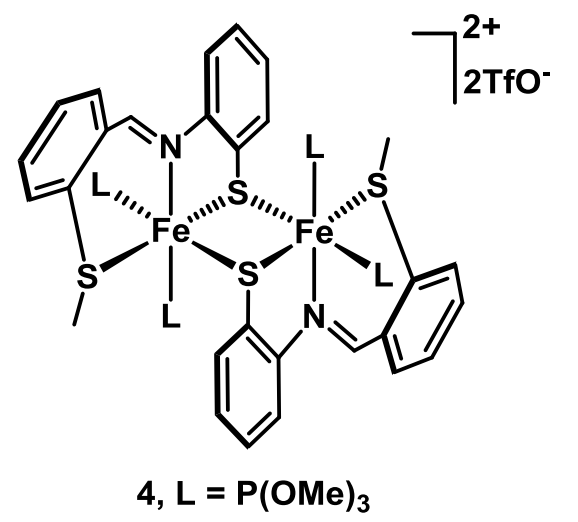

กิ

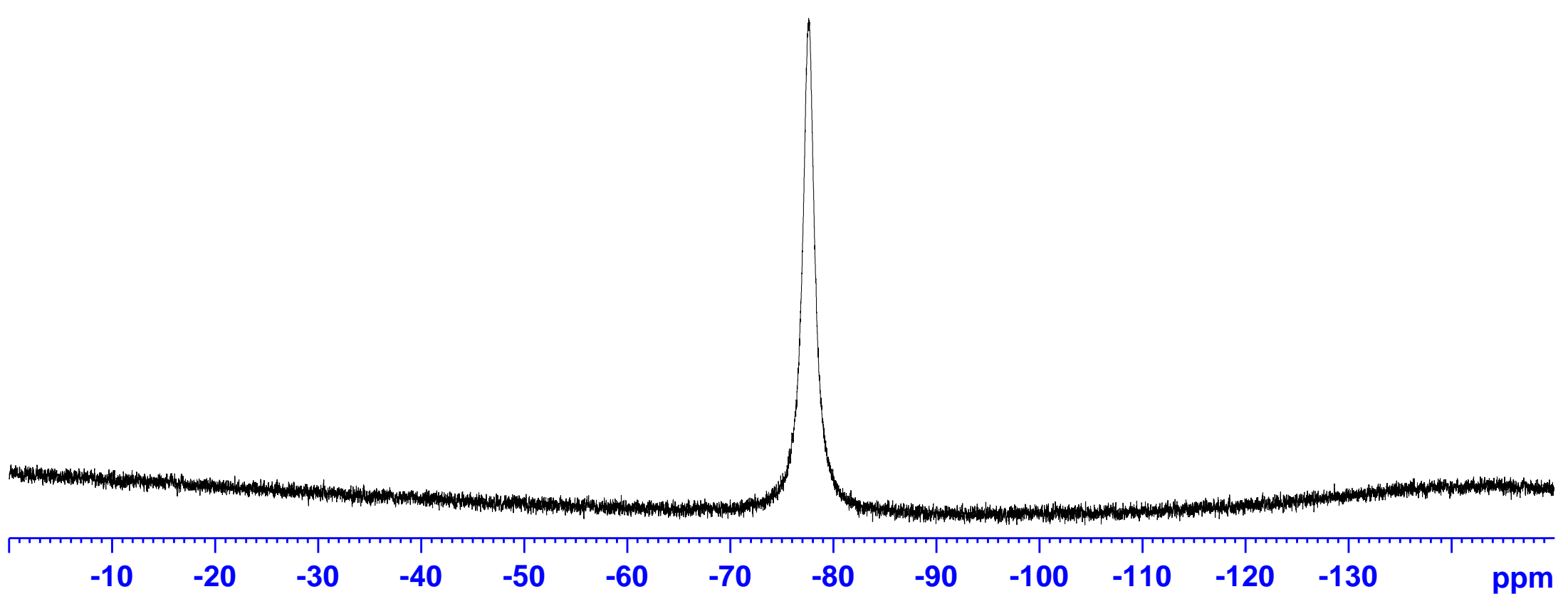

Figure S22. ${ }^{19} \mathrm{~F}$ NMR spectrum $\left(282 \mathrm{MHz}, \mathrm{CD}_{3} \mathrm{CN}\right)$ of $\left\{\left[\mathrm{Fe}\left(\mu-\mathrm{S}^{M e} \mathrm{NS}^{-}\right)\left[\mathrm{P}(\mathrm{OMe})_{3}\right]_{2}\right]_{2}\right\}(\mathrm{OTf})_{2}(4)$. $(\mathrm{LB}=3 \mathrm{~Hz})$. 


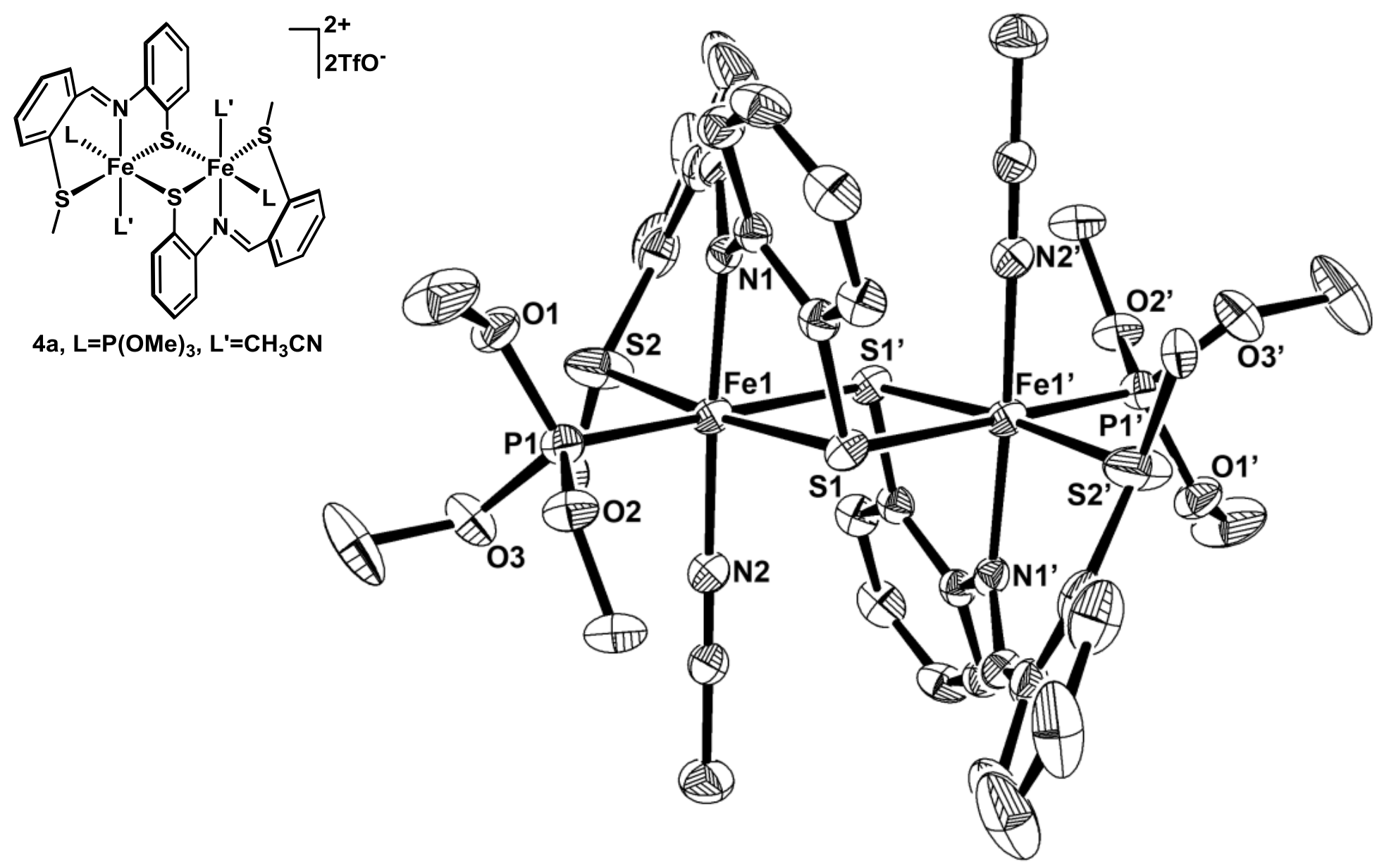

Figure S23. ORTEP diagram of $\left\{\left[\mathrm{Fe}\left(\mu-\mathrm{S}^{M e} \mathrm{NS}^{-}\right)\left(\mathrm{CH}_{3} \mathrm{CN}\right)\left[\mathrm{P}(\mathrm{OMe})_{3}\right]\right]_{2}\right\}(\mathrm{OTf})_{2}(\mathbf{4 a})$. Thermal ellipsoids are shown at $40 \%$ probability. Hydrogen atoms, $\mathrm{CH}_{3} \mathrm{CN}$ molecules and triflate anions are omitted for clarity. Selected bond lengths and angles are given in Tables 2 and 3. 

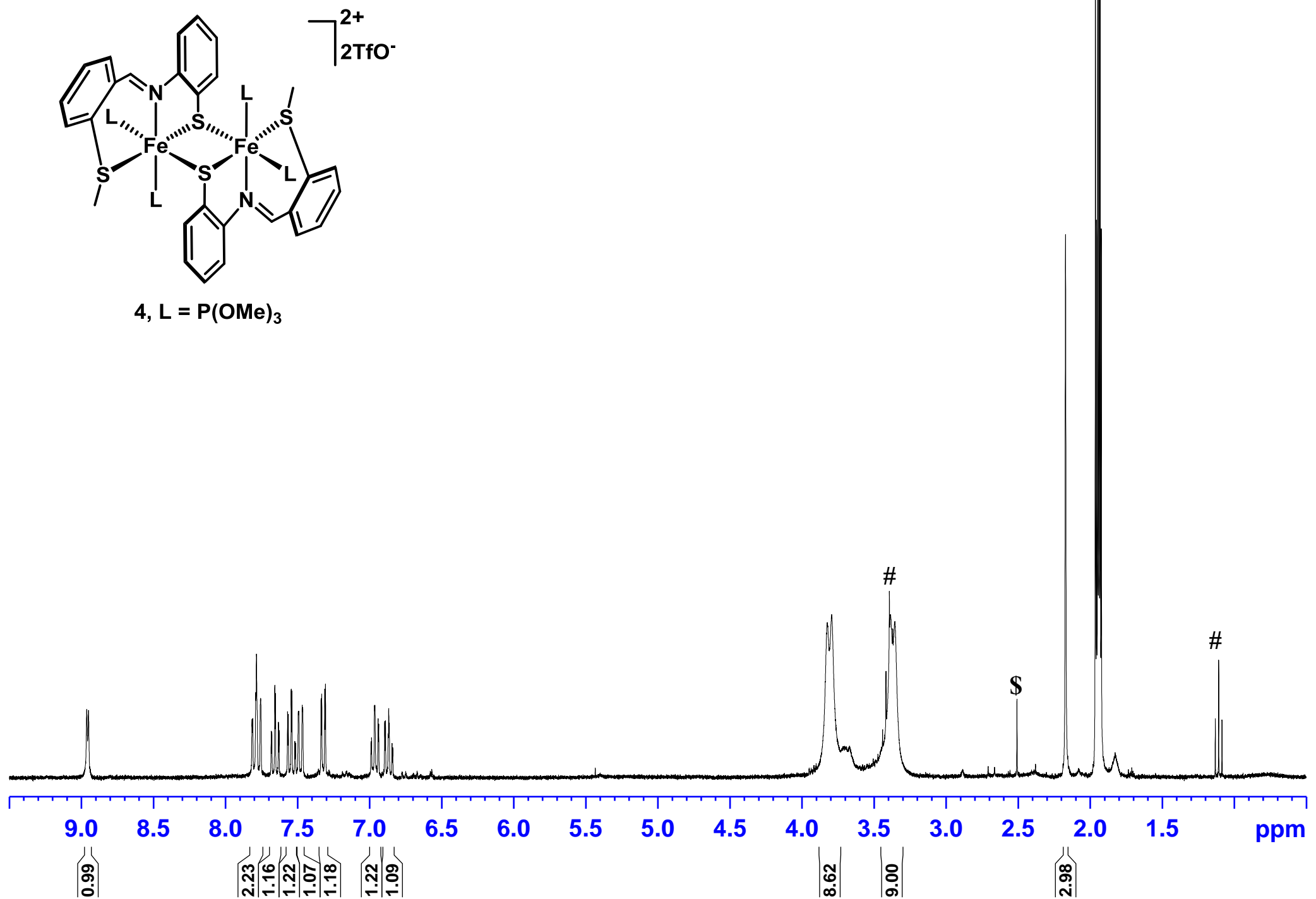

Figure S24. ${ }^{1} \mathrm{H}$ NMR spectrum $\left(300 \mathrm{MHz}, \mathrm{CD}_{3} \mathrm{CN}\right)$ of $\left\{\left[\mathrm{Fe}\left(\mu-\mathrm{S}^{M e} \mathrm{NS}^{-}\right)\left(\mathrm{CH}_{3} \mathrm{CN}\right)\left[\mathrm{P}(\mathrm{OMe})_{3}\right]\right]_{2}\right\}(\mathrm{OTf})_{2}(\mathbf{4 a})$. [*NMR solvent; ${ }^{*}$ diethyl ether; ${ }^{\$}$ impurity] 

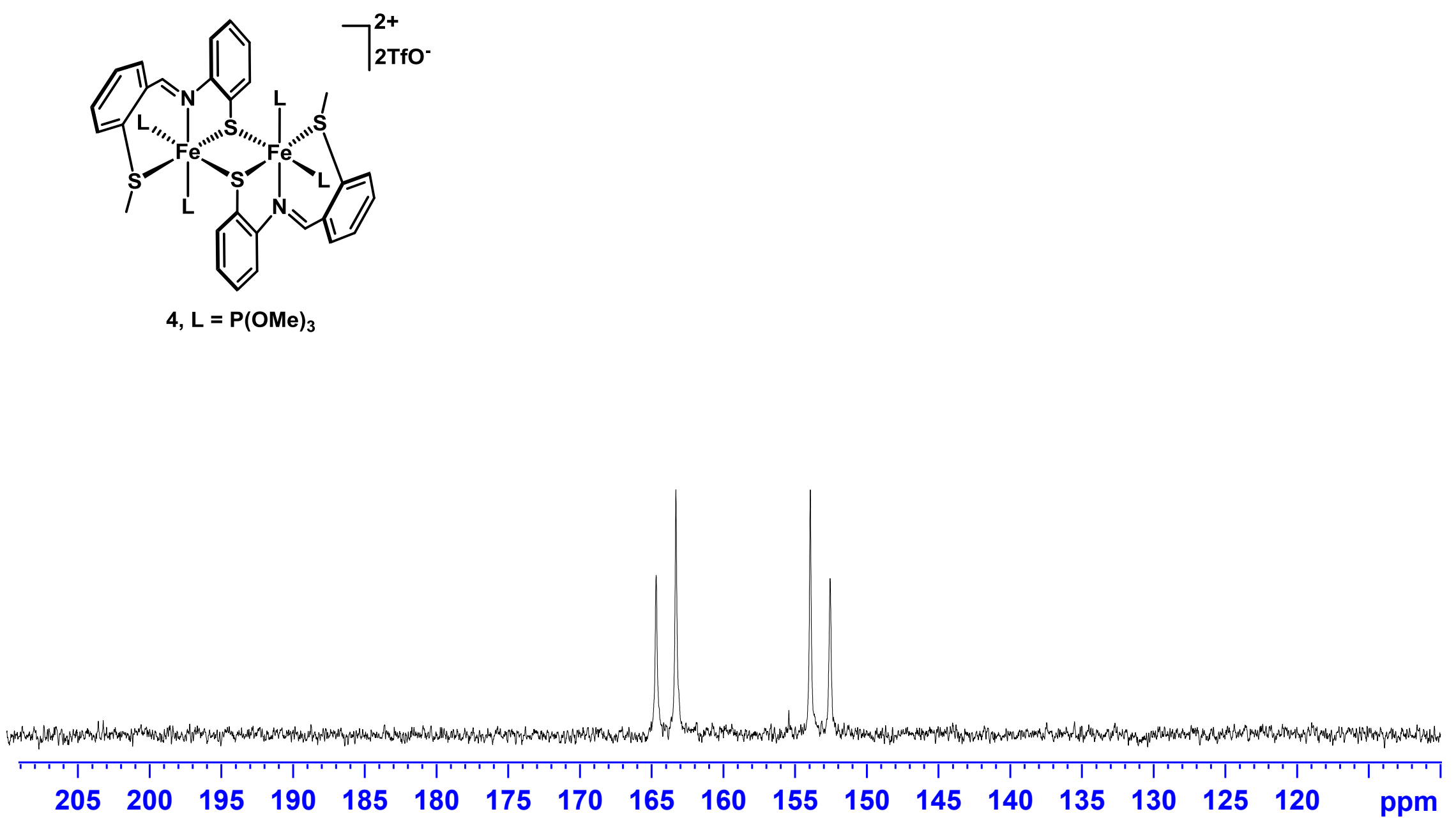

Figure S25. ${ }^{31} \mathrm{P}\left\{{ }^{1} \mathrm{H}\right\}$ NMR spectrum $\left(121 \mathrm{MHz}, \mathrm{CD}_{3} \mathrm{CN}\right)$ of $\left\{\left[\mathrm{Fe}\left(\mu-\mathrm{S}^{\mathrm{Me}} \mathrm{NS}^{-}\right)\left(\mathrm{CH}_{3} \mathrm{CN}\right)\left[\mathrm{P}(\mathrm{OMe})_{3}\right]\right]_{2}\right\}(\mathrm{OTf})_{2}(\mathbf{4 a})$. 


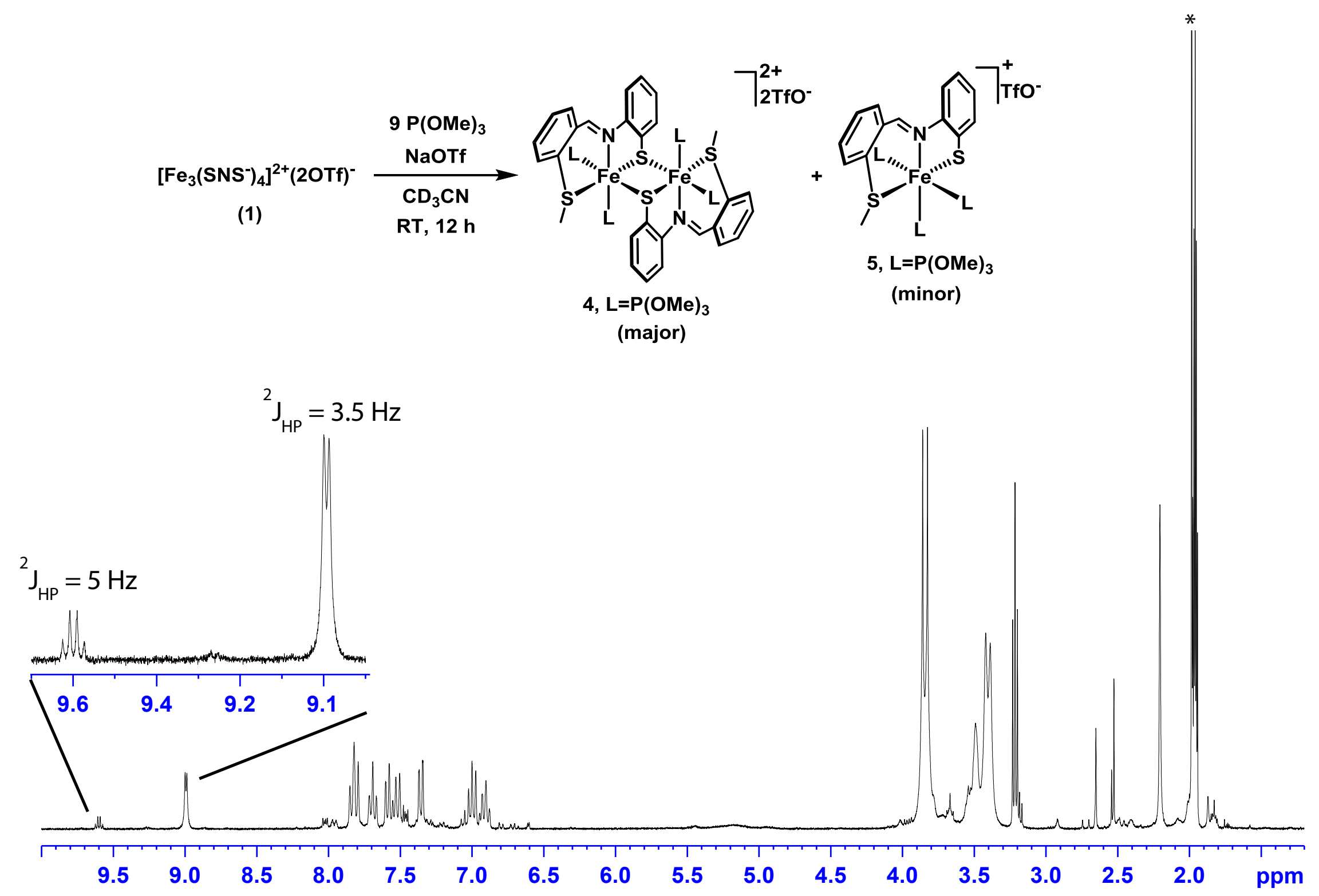

Figure S26. ${ }^{1} \mathrm{H}$ NMR spectrum $\left(300 \mathrm{MHz}, \mathrm{CD}_{3} \mathrm{CN}\right)$ of the reaction of $\left[\mathrm{Fe}_{3}\left(\mu_{2}-\mathrm{S}^{M e} \mathrm{NS}^{-}\right)_{4}\right](\mathrm{OTf})_{2}(\mathbf{1})$ and NaOTf with excess $\mathrm{P}(\mathrm{OMe})_{3}$. 


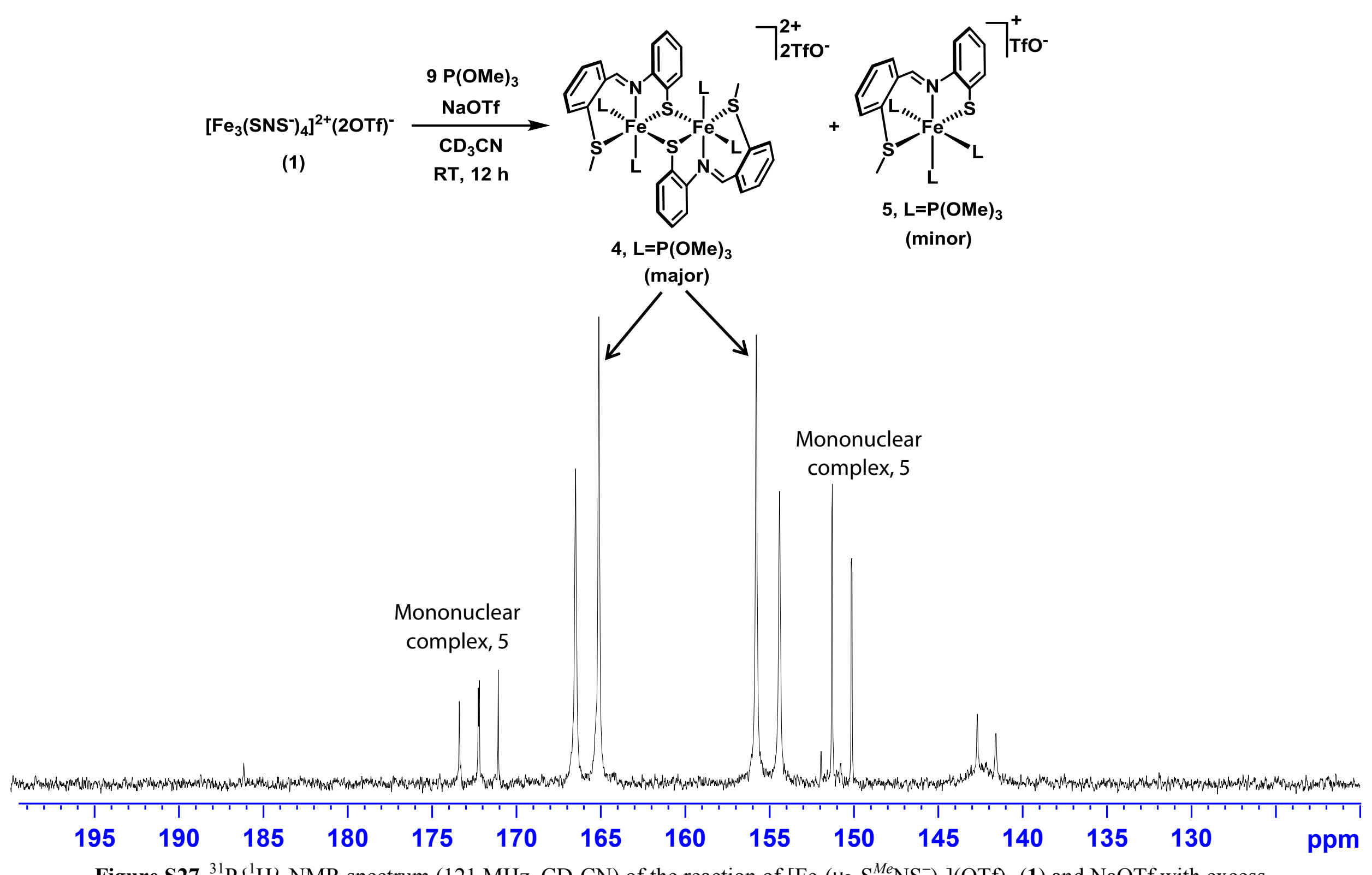

Figure S27. ${ }^{31} \mathrm{P}\left\{{ }^{1} \mathrm{H}\right\}$ NMR spectrum $\left(121 \mathrm{MHz}, \mathrm{CD}_{3} \mathrm{CN}\right)$ of the reaction of $\left[\mathrm{Fe}_{3}\left(\mu_{2}-\mathrm{S}^{M e} \mathrm{NS}^{-}\right)_{4}\right](\mathrm{OTf})_{2}(\mathbf{1})$ and NaOTf with excess $\mathrm{P}(\mathrm{OMe})_{3}$. 


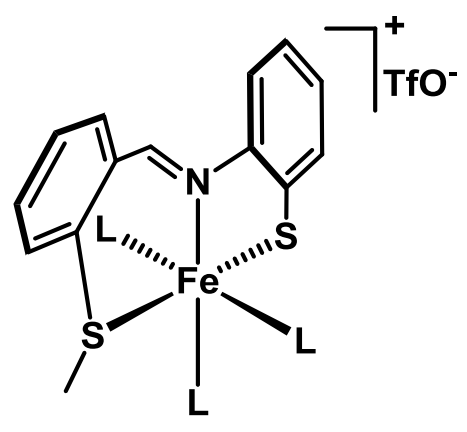

5, $\mathrm{L}=\mathrm{P}(\mathrm{OMe})_{3}$

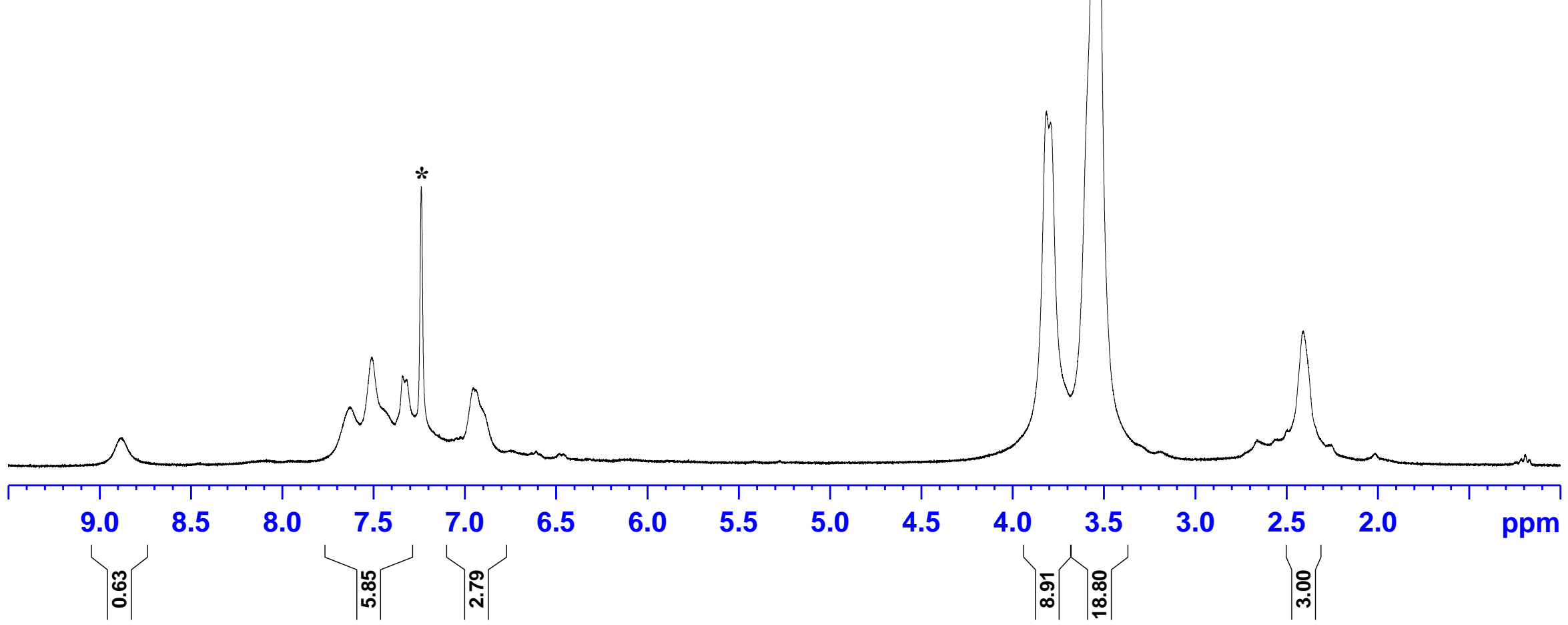

Figure S28. ${ }^{1} \mathrm{H}$ NMR spectrum $\left(300 \mathrm{MHz}, \mathrm{CDCl}_{3}\right)$ of $\left[\mathrm{Fe}\left(\mathrm{S}^{M e} \mathrm{NS}^{-}\right)\left\{\mathrm{P}(\mathrm{OMe})_{3}\right\}_{3}\right](\mathrm{OTf})(\mathbf{5})$. [*residual $\mathrm{NMR}$ solvent $]$ 


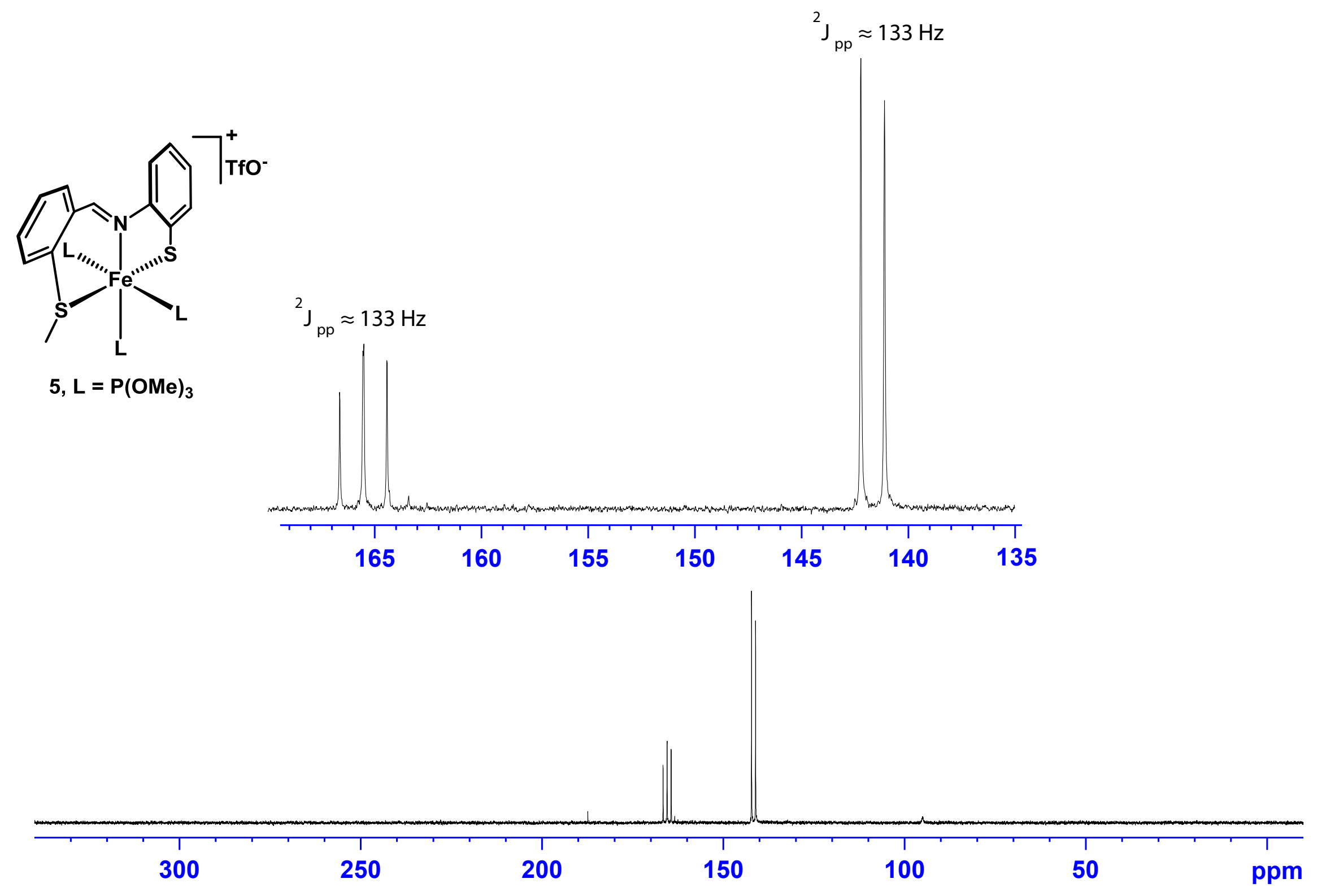

Figure S29. ${ }^{31} \mathrm{P}\left\{{ }^{1} \mathrm{H}\right\} \mathrm{NMR}$ spectrum $\left(121 \mathrm{MHz}, \mathrm{CDCl}_{3}\right)$ of $\left[\mathrm{Fe}\left(\mathrm{S}^{M e} \mathrm{NS}^{-}\right)\left\{\mathrm{P}(\mathrm{OMe})_{3}\right\}_{3}\right](\mathrm{OTf})(\mathbf{5})$. 


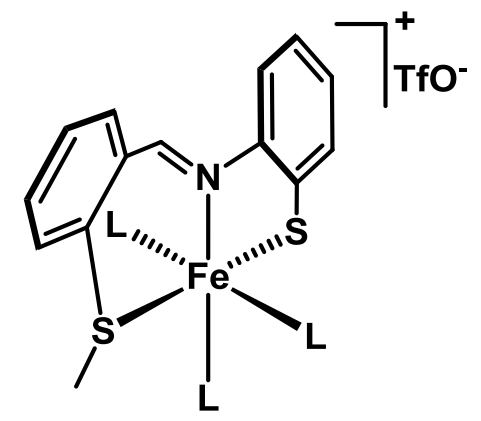

$5, \mathrm{~L}=\mathrm{P}(\mathrm{OMe})_{3}$

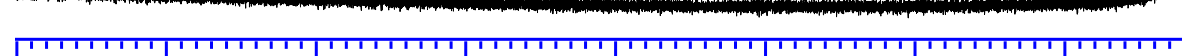

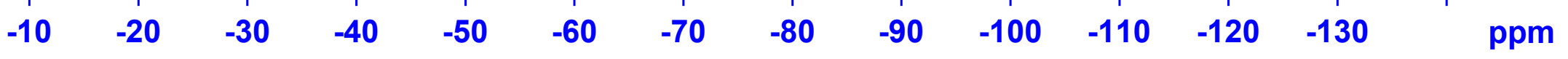

Figure S30. ${ }^{19} \mathrm{~F}$ NMR spectrum $\left(282 \mathrm{MHz}, \mathrm{CDCl}_{3}\right)$ of $\left[\mathrm{Fe}\left(\mathrm{S}^{M e} \mathrm{NS}^{-}\right)\left\{\mathrm{P}(\mathrm{OMe})_{3}\right\}_{3}\right](\mathrm{OTf})(\mathbf{5})$. 


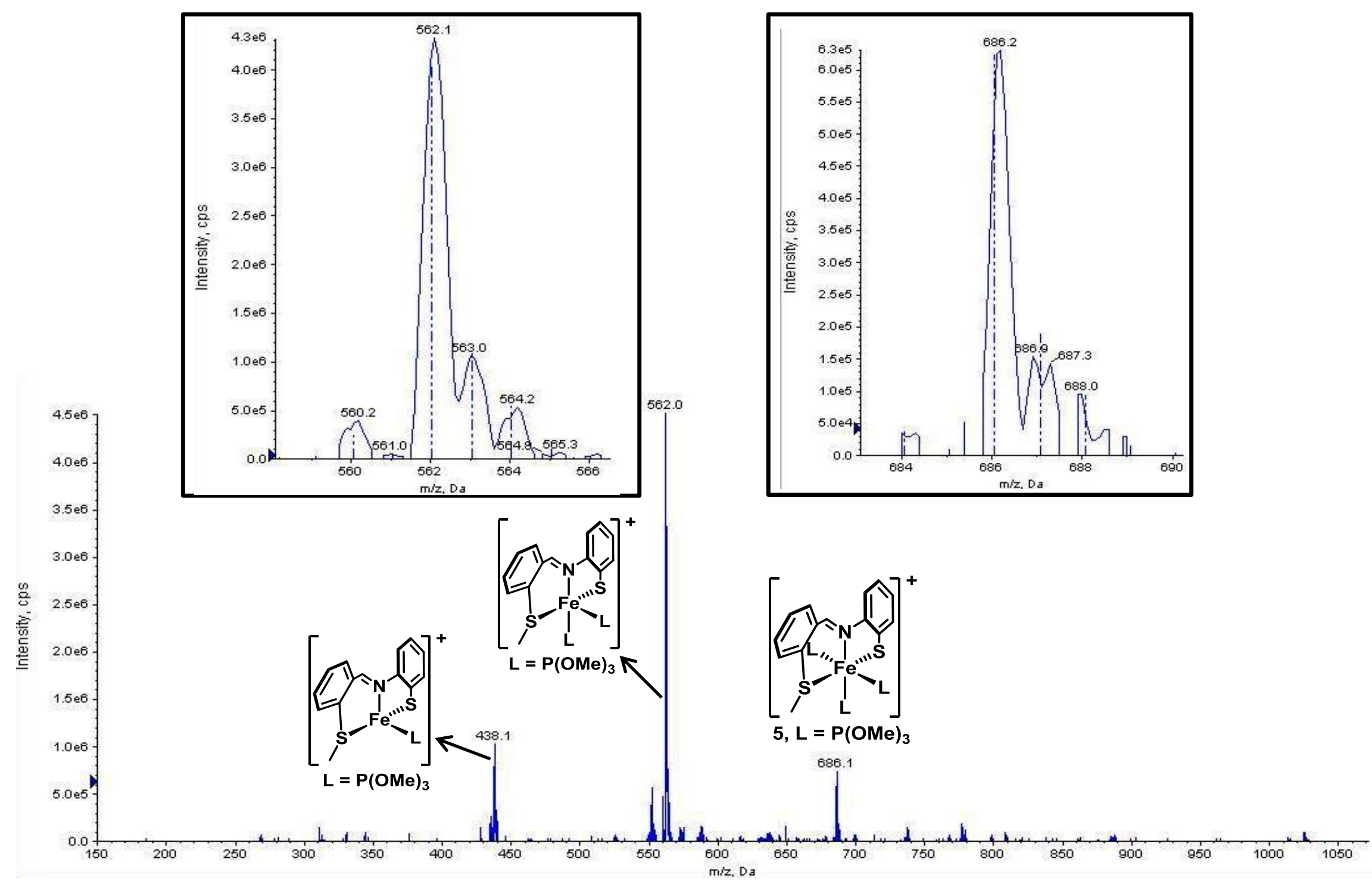

Figure S31. ESI-MS spectrum of $\left[\mathrm{Fe}\left(\mathrm{S}^{M e} \mathrm{NS}^{-}\right)\left\{\mathrm{P}(\mathrm{OMe})_{3}\right\}_{3}\right](\mathrm{OTf})(\mathbf{5})$. Inset figures: comparison of experimental isotopic patterns of $[\mathrm{M}]^{+}=686.1$ and 562.0 with simulated isotopic patterns. 
Table S1. Energies and MPA-derived compositions of the frontier molecular orbitals of $\left[\mathrm{Fe}\left(\mathrm{S}^{M e} \mathrm{NS}{ }^{-}\right)\left\{\mathrm{P}(\mathrm{OMe})_{3}\right\}_{3}\right](\mathrm{OTf})(\mathbf{5})($ at the B3LYP/TZVP level).

\begin{tabular}{|l|l|l|l|l|}
\hline & \multirow{2}{*}{} & E (eV) & \multicolumn{3}{l}{ Composition (\%) } \\
\cline { 3 - 5 } & & Fe & $\mathrm{S}^{M e} \mathrm{NS}^{-}$ligand & Phosphite ligands \\
\hline LUMO+3 & -1.43 & 57 & 16 & 27 \\
\hline LUMO+2 & -1.50 & 2 & 95 & 3 \\
\hline LUMO+1 & -1.62 & 58 & 23 & 19 \\
\hline LUMO & -2.97 & 3 & 96 & 1 \\
\hline HOMO & -5.64 & 11 & 85 & 4 \\
\hline HOMO-1 & -6.87 & 52 & 45 & 3 \\
\hline HOMO-2 & -7.06 & 35 & 61 & 5 \\
\hline HOMO-3 & -7.29 & 63 & 26 & 11 \\
\hline HOMO-4 & -7.44 & 48 & 41 & 11 \\
\hline
\end{tabular}


Table S2. TD-DFT-calculated electronic transitions of $\left[\mathrm{Fe}\left(\mathrm{S}^{M e} \mathrm{NS}^{-}\right)\left\{\mathrm{P}(\mathrm{OMe})_{3}\right\}_{3}\right](\mathrm{OTf})(\mathbf{5})$ (the HOMO is orbital \#179 and the LUMO is orbital \#180):

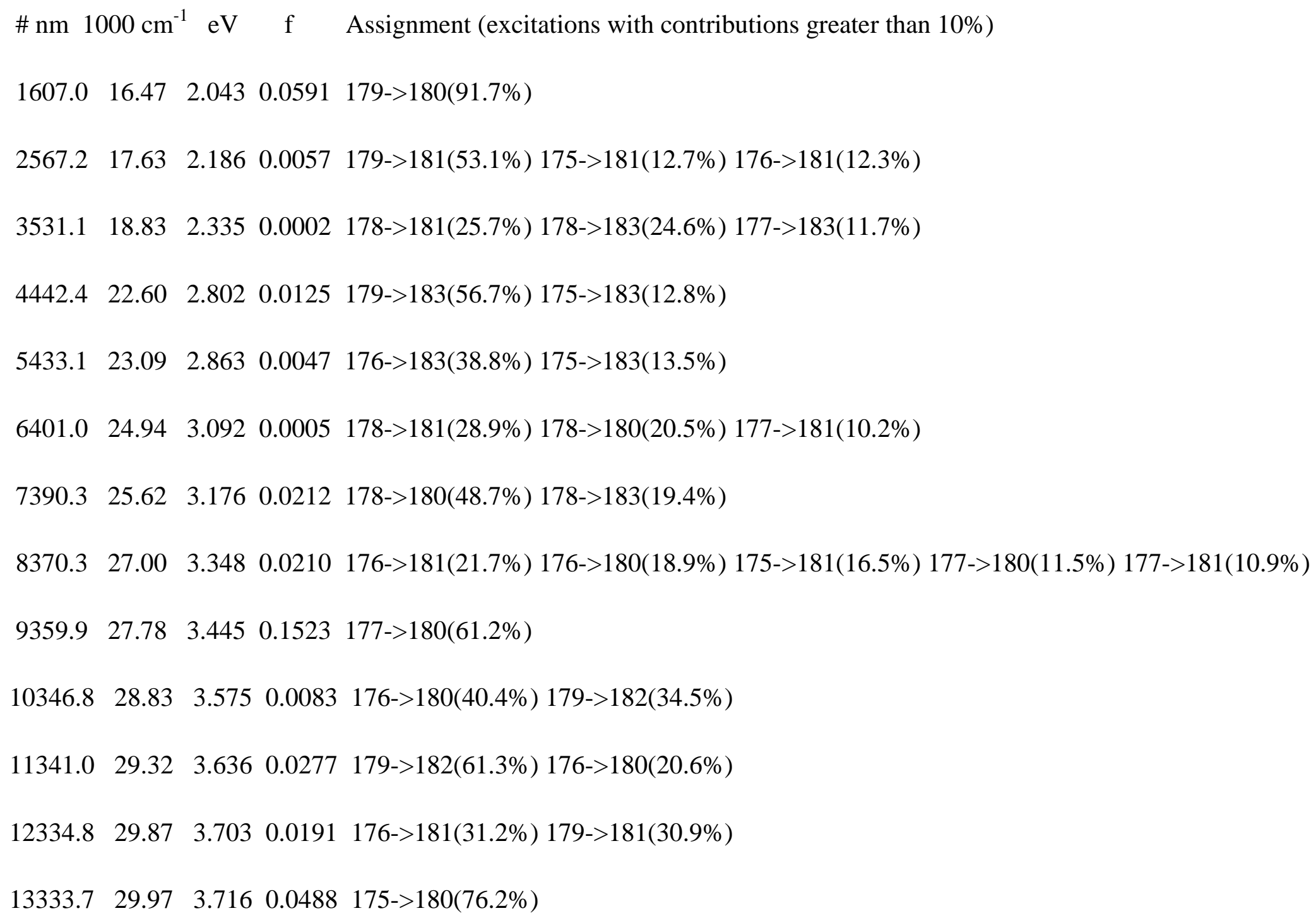




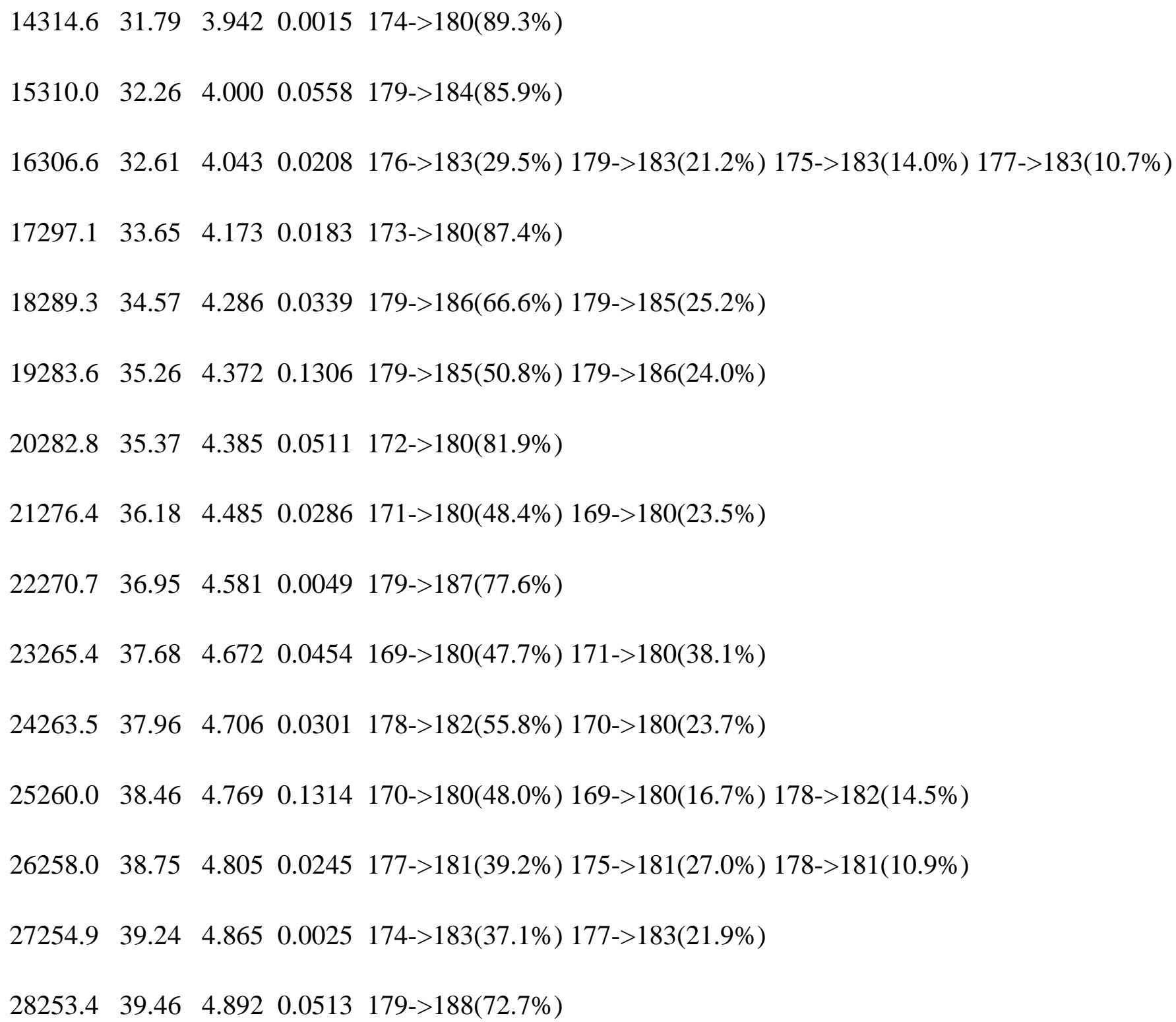




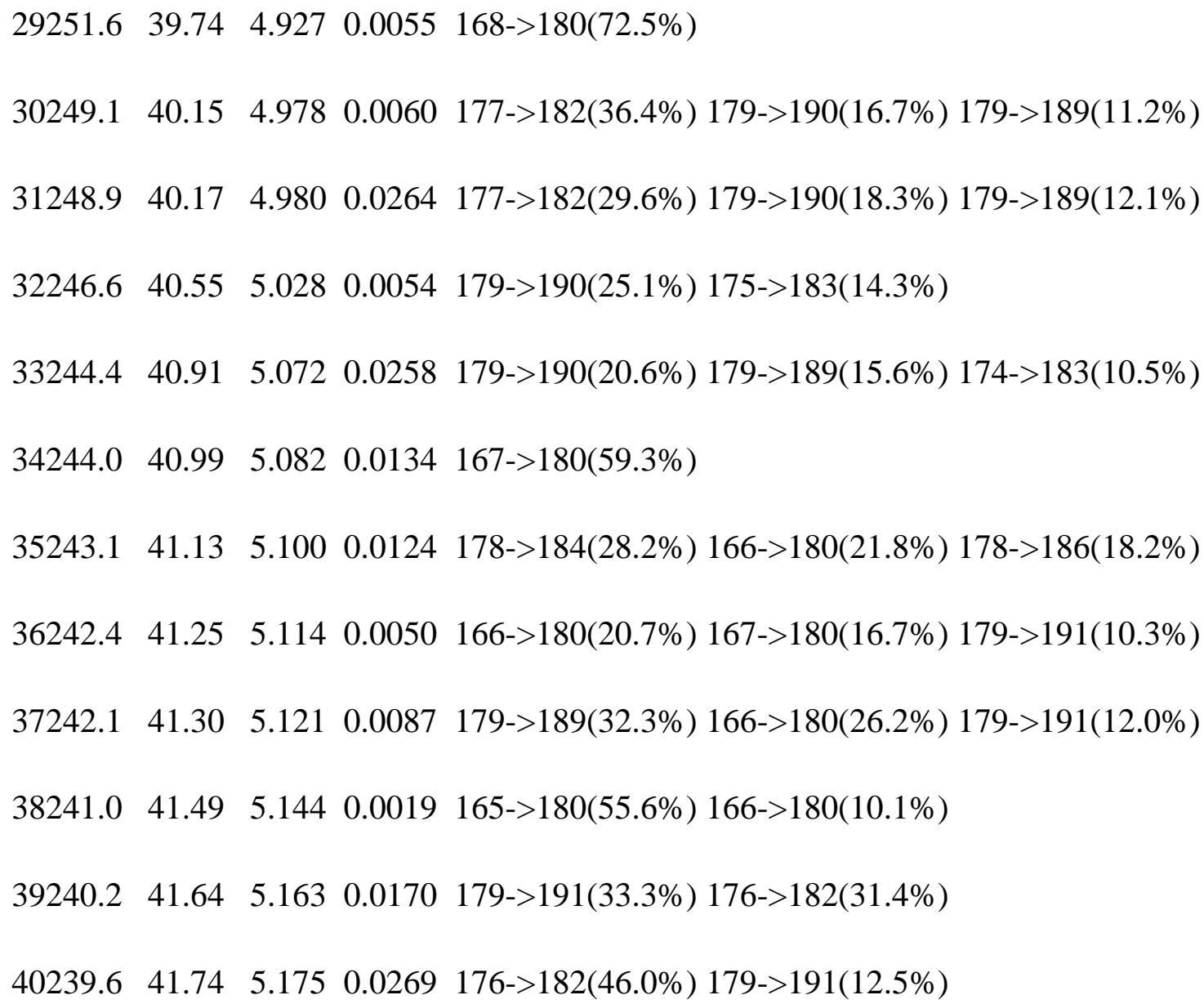




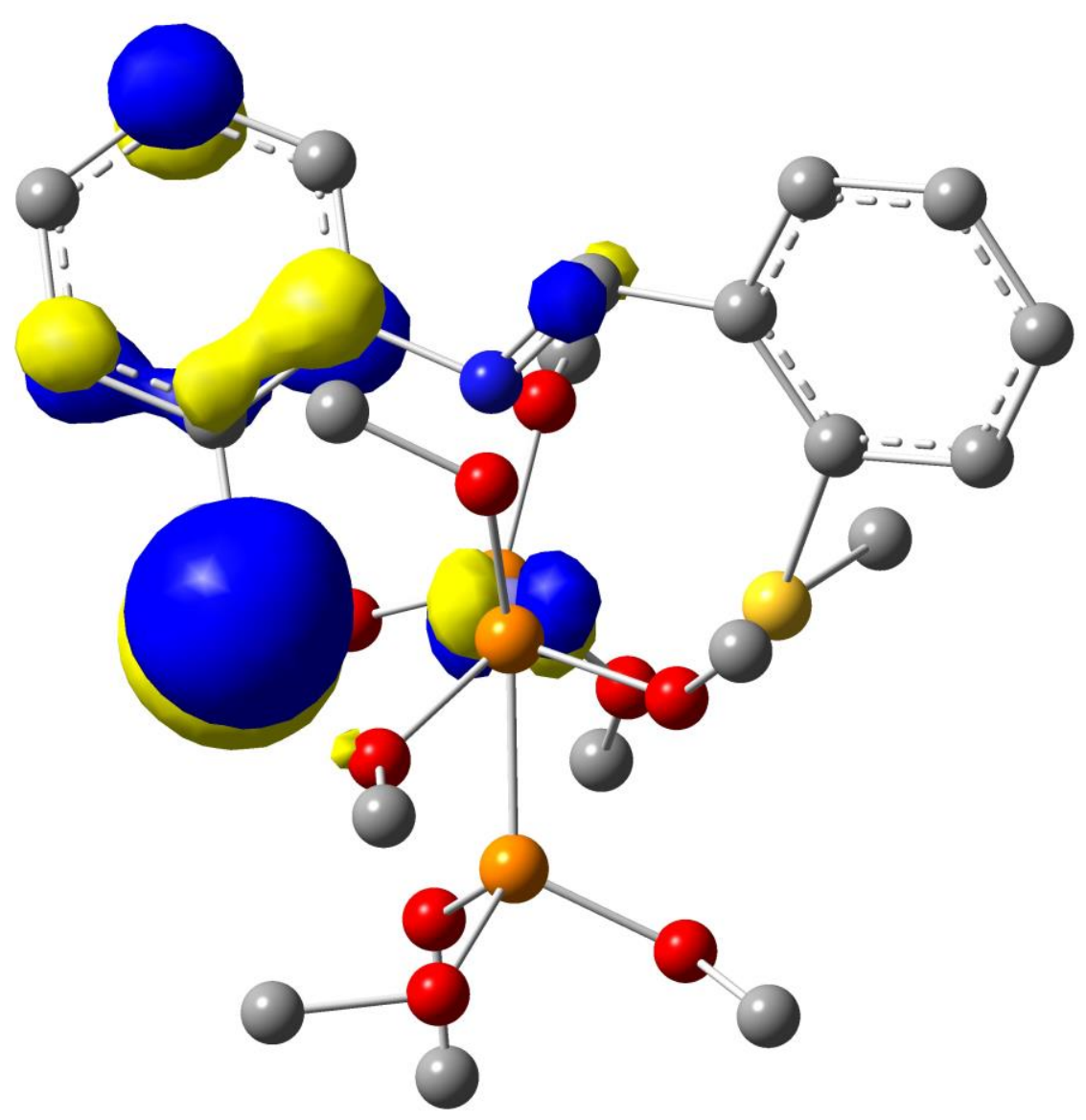

Figure S32. The HOMO of $\left[\mathrm{Fe}\left(\mathrm{S}^{M e} \mathrm{NS}^{-}\right)\left\{\mathrm{P}(\mathrm{OMe})_{3}\right\}_{3}\right](\mathrm{OTf})(\mathbf{5})$. The orbital is shown with the isosurface value of 0.05 a.u. $\mathrm{H}$ atoms are omitted for clarity. 


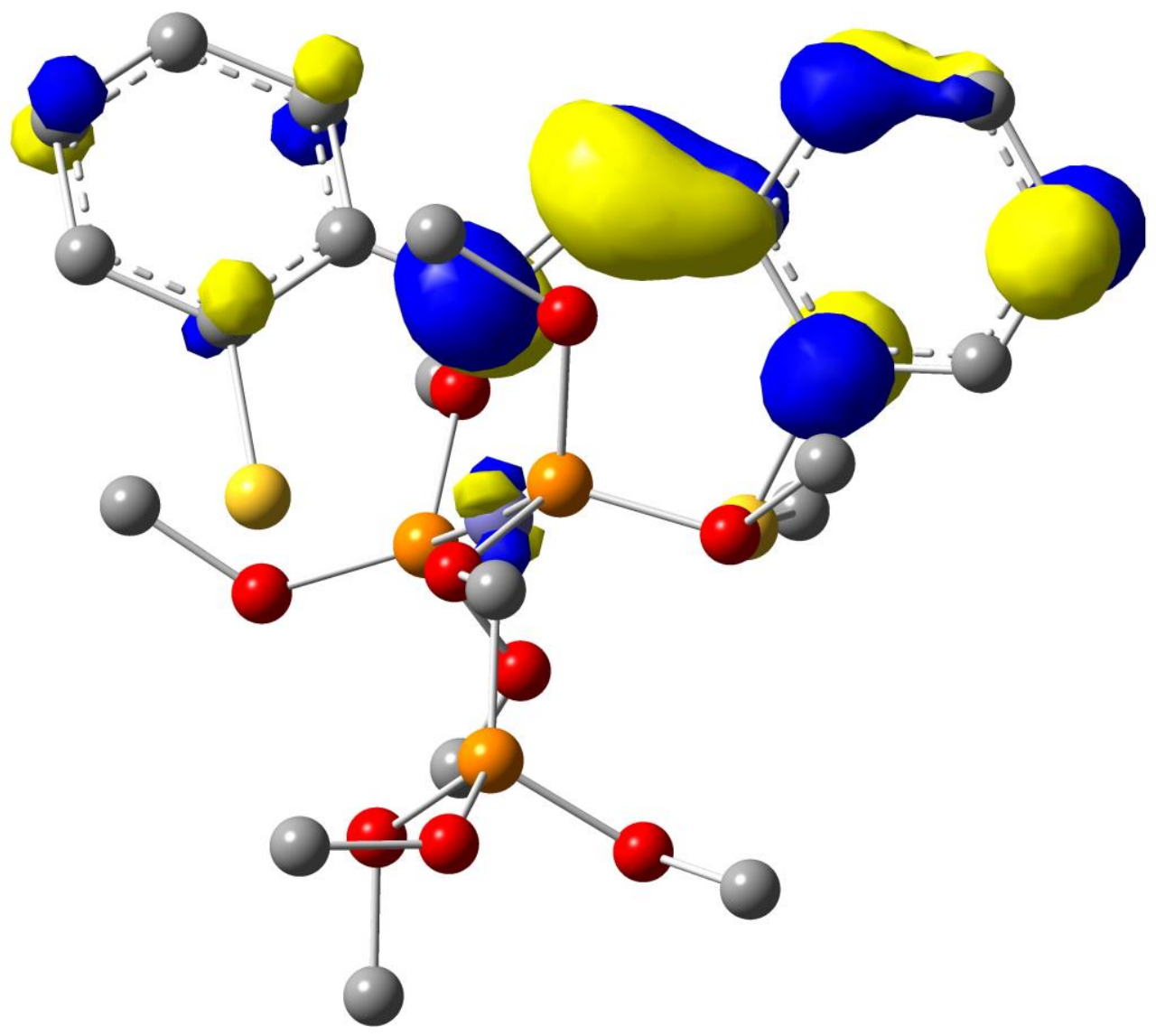

Figure S33. The LUMO of $\left[\mathrm{Fe}\left(\mathrm{S}^{M e} \mathrm{NS}^{-}\right)\left\{\mathrm{P}(\mathrm{OMe})_{3}\right\}_{3}\right](\mathrm{OTf})(\mathbf{5})$. The orbital is shown with the isosurface value of 0.05 a.u. $\mathrm{H}$ atoms are omitted for clarity. 


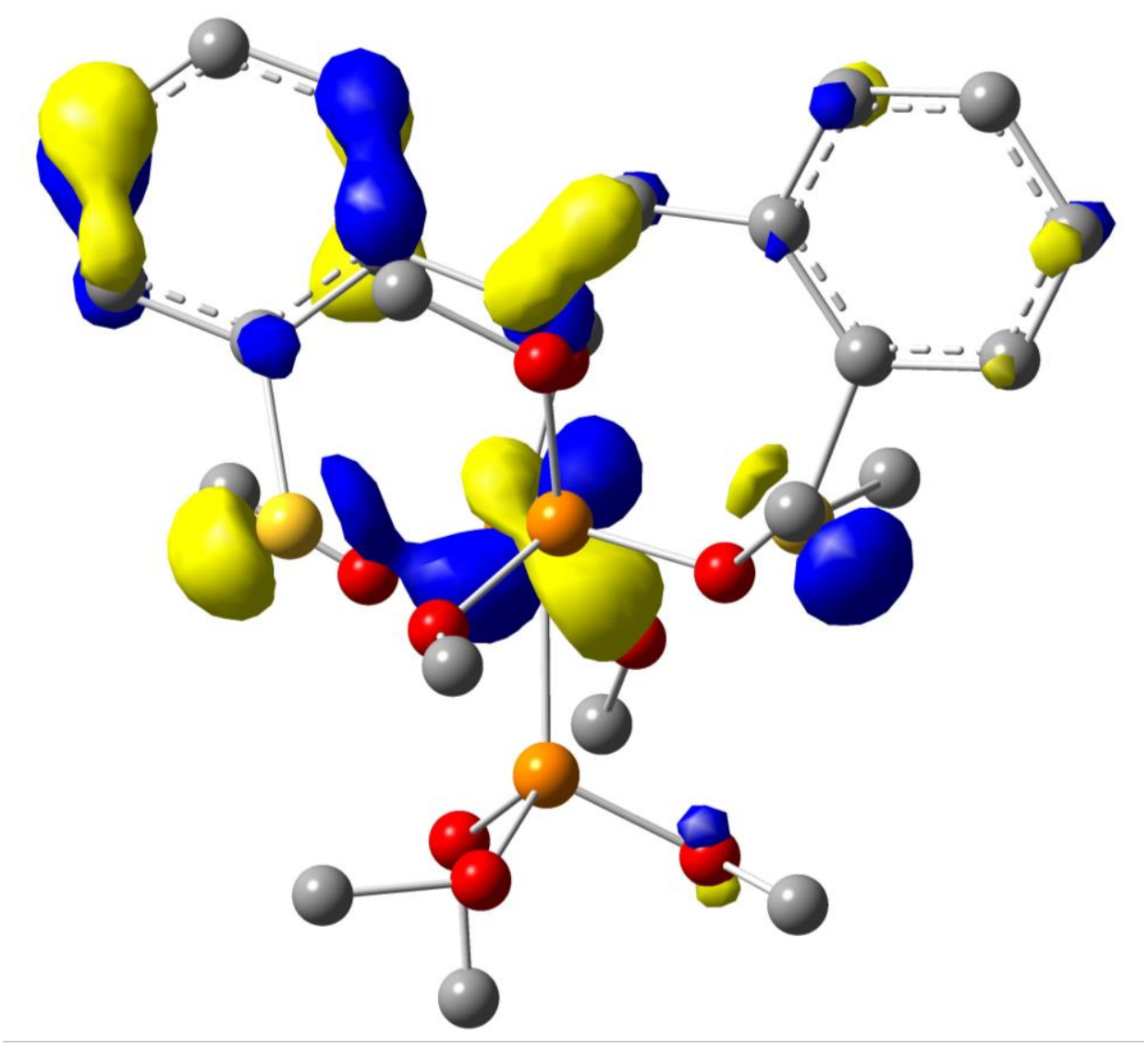

Figure S34. The HOMO-2 of $\left[\mathrm{Fe}\left(\mathrm{S}^{M e} \mathrm{NS}^{-}\right)\left\{\mathrm{P}(\mathrm{OMe})_{3}\right\}_{3}\right](\mathrm{OTf})(\mathbf{5})$. The orbital is shown with the isosurface value of 0.05 a.u. $\mathrm{H}$ atoms are omitted for clarity. 


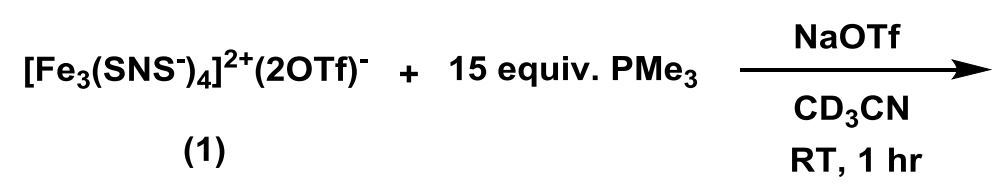

(1)

\section{RT, 1 hr}

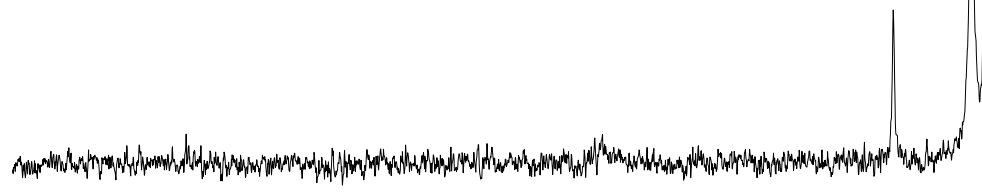

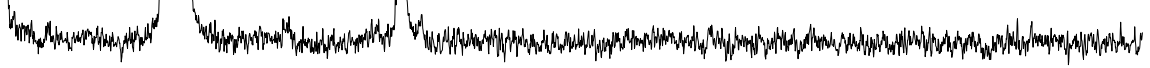
45

Figure S35. ${ }^{31} \mathrm{P}\left\{{ }^{1} \mathrm{H}\right\} \mathrm{NMR}$ spectrum $\left(121 \mathrm{MHz}, \mathrm{CD}_{3} \mathrm{CN}\right)$ of the reaction of $\left[\mathrm{Fe}_{3}\left(\mu_{2}-\mathrm{S}^{M e} \mathrm{NS}^{-}\right)_{4}\right](\mathrm{OTf})_{2}(\mathbf{1})$ and $\mathrm{NaOTf}_{\text {with } \mathrm{PMe}}$. 

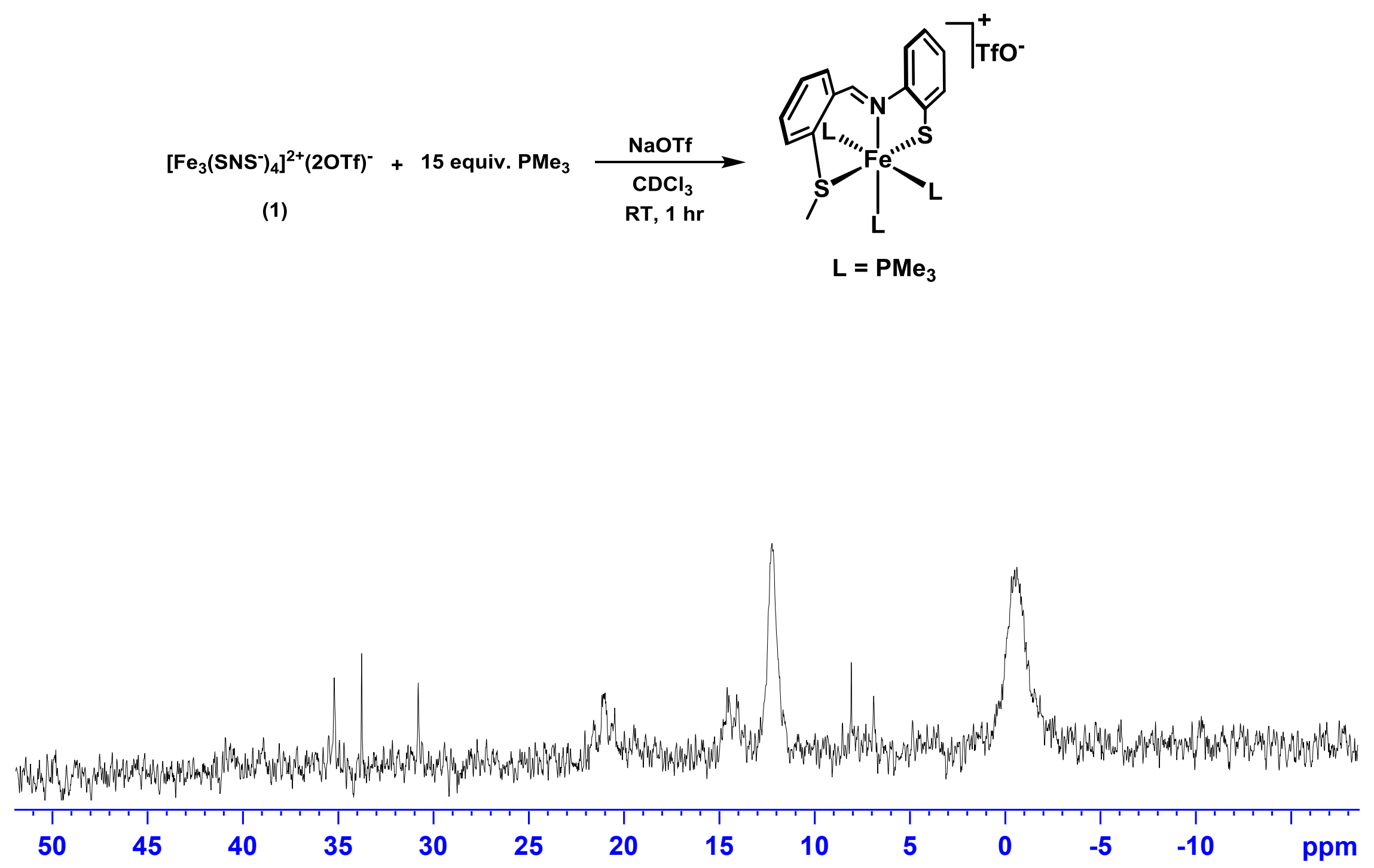

Figure S36. ${ }^{31} \mathrm{P}\left\{{ }^{1} \mathrm{H}\right\}$ NMR spectrum $\left(121 \mathrm{MHz}, \mathrm{CDCl}_{3}\right)$ of the reaction of $\left[\mathrm{Fe}_{3}\left(\mu_{2}-\mathrm{S}^{\mathrm{Me}} \mathrm{NS}^{-}\right)_{4}\right](\mathrm{OTf})_{2}(\mathbf{1})$ and $\mathrm{NaOTf}$ with excess $\mathrm{PMe}_{3}$. 

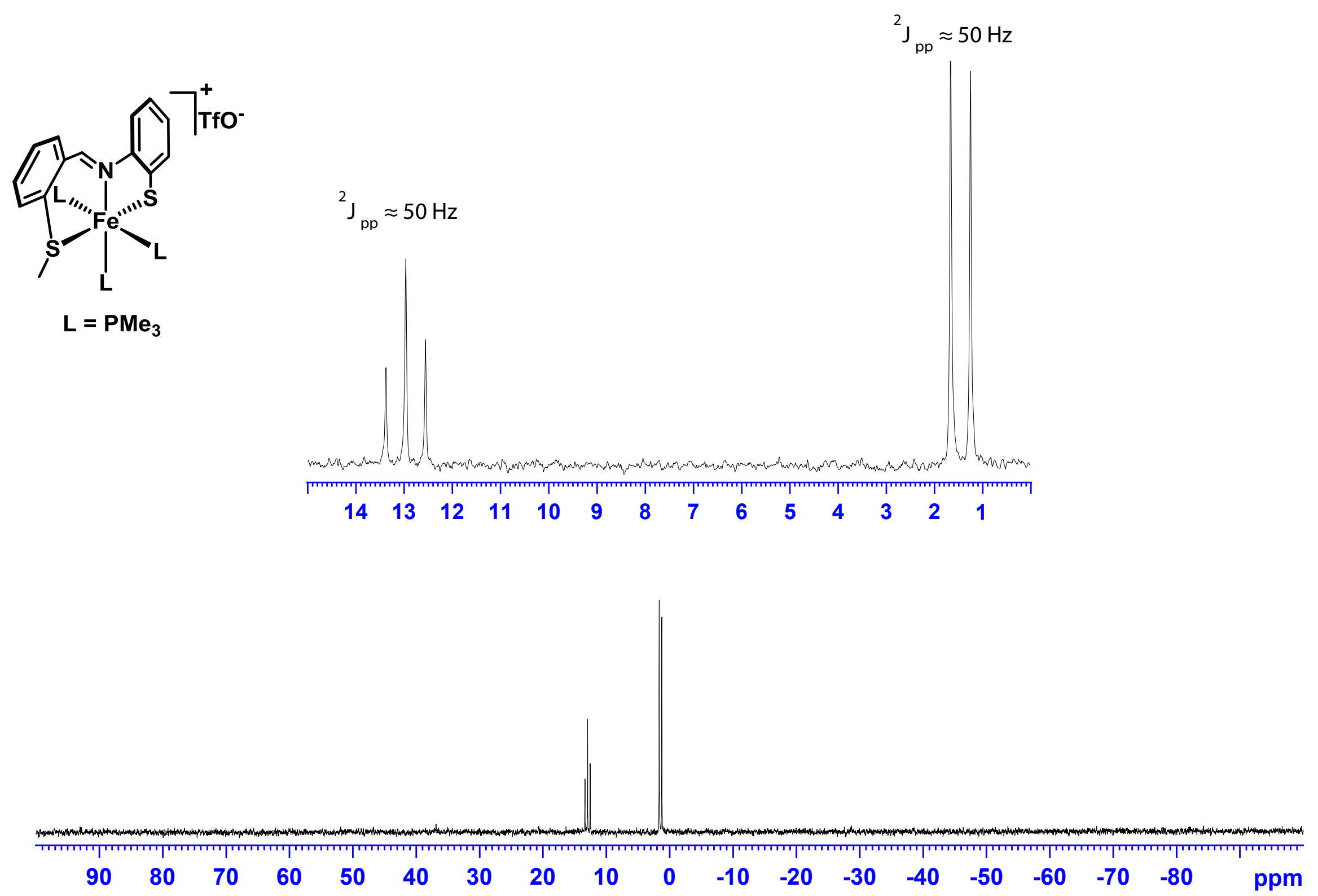

Figure S37. ${ }^{31} \mathrm{P}\left\{{ }^{1} \mathrm{H}\right\}$ NMR spectrum $\left\{121 \mathrm{MHz},\left(\mathrm{CD}_{3}\right)_{2} \mathrm{CO}\right\}$ of the reaction of $\left[\mathrm{Fe}_{3}\left(\mu_{2}-\mathrm{S}^{M e} \mathrm{NS}^{-}\right)_{4}\right](\mathrm{OTf})_{2}(\mathbf{1})$ and NaOTf with excess $\mathrm{PMe}_{3}$ at $-20^{\circ} \mathrm{C}$. 


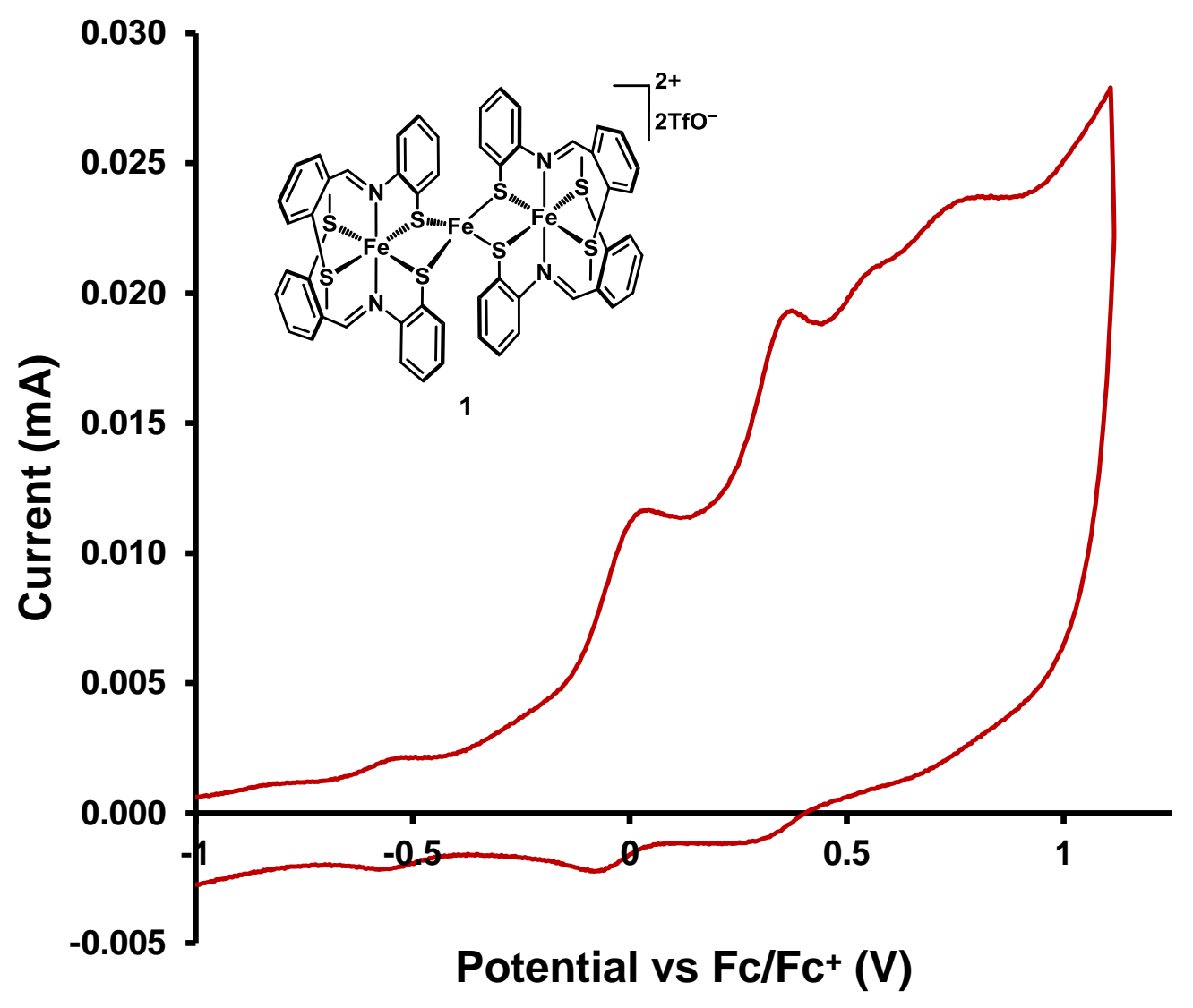

\begin{tabular}{|ccc|}
\hline $\mathbf{E}_{\mathbf{p}}{ }^{\mathbf{a}}$ & $\mathbf{E}_{\mathbf{p}}{ }^{\mathbf{c}}$ & $\mathbf{E}_{\mathbf{1} / \mathbf{2}}$ \\
\hline 0.012 & -0.072 & -0.03 \\
\hline 0.361 & 0.295 & 0.33 \\
\hline
\end{tabular}

Figure S38. Cyclic voltammogram of $\left[\mathrm{Fe}_{3}\left(\mu_{2}-\mathrm{S}^{M e} \mathrm{NS}^{-}\right)_{4}\right](\mathrm{OTf})_{2}(\mathbf{1})$ in $\mathrm{CH}_{2} \mathrm{Cl}_{2}$ under $\mathrm{N}_{2}$ atmosphere ([complex] $0.5 \mathrm{mM}$, at $100 \mathrm{mV}$ scan rate in $0.1 \mathrm{M} \mathrm{n}-\mathrm{Bu}_{4} \mathrm{NPF}_{6}$ ). 

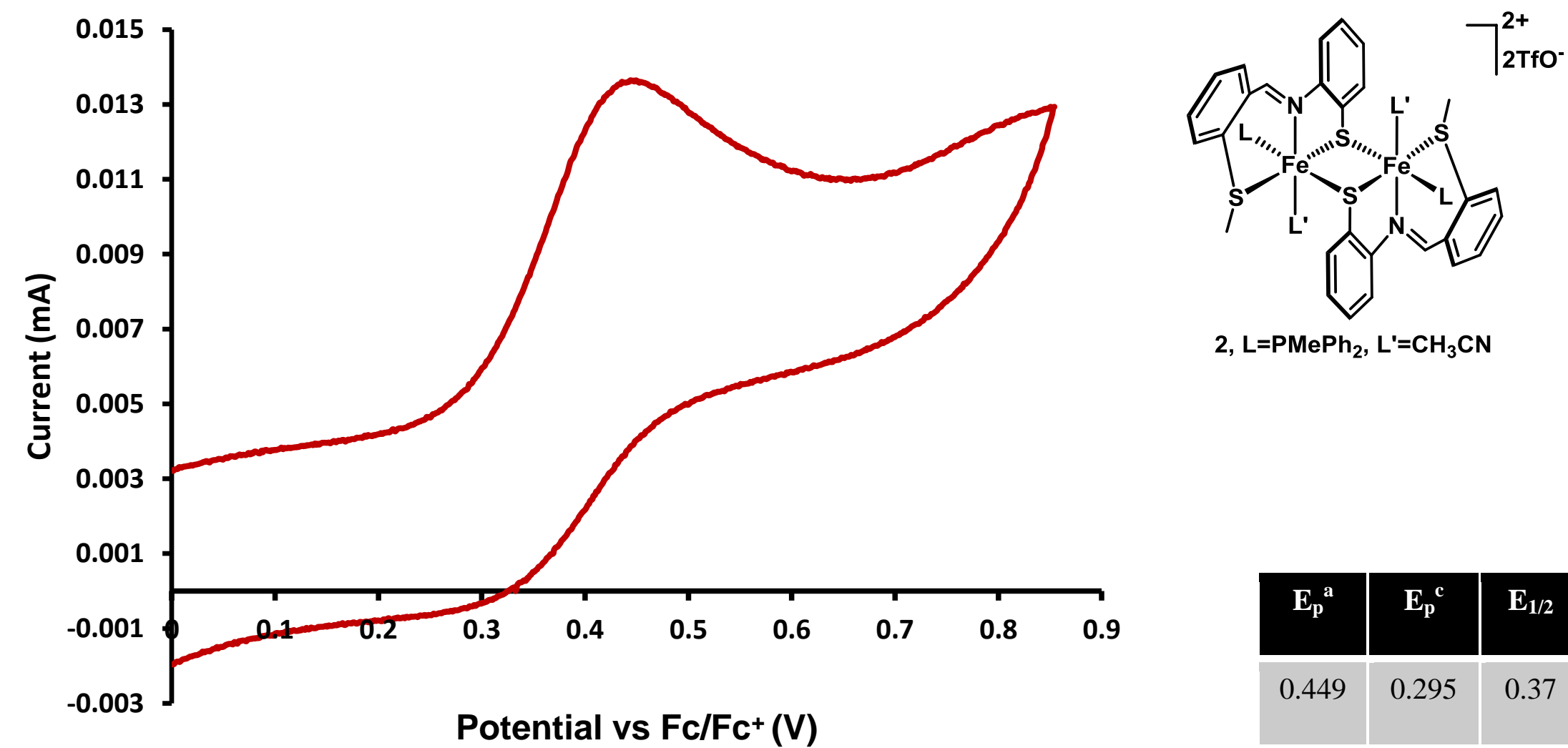

Figure S39. Cyclic voltammogram of $\left\{\left[\mathrm{Fe}\left(\mu-\mathrm{S}^{M e} \mathrm{NS}^{-}\right)\left(\mathrm{PMePh}_{2}\right)\left(\mathrm{CH}_{3} \mathrm{CN}\right)\right]_{2}\right\}(\mathrm{OTf})_{2}(\mathbf{2})$ in $\mathrm{CH}_{2} \mathrm{Cl}_{2}$ under $\mathrm{N}_{2}$ atmosphere ([complex] 0.5 $\mathrm{mM}$, at $100 \mathrm{mV}$ scan rate in $0.1 \mathrm{M} \mathrm{n}-\mathrm{Bu}_{4} \mathrm{NPF}_{6}$ ). 


\section{Experimental Section}

Mössbauer Spectroscopy. All solid samples for ${ }^{57} \mathrm{Fe}$ Mössbauer spectroscopy were run on nonenriched samples of the as-isolated complexes. All samples were prepared in an inert atmosphere glovebox equipped with a liquid nitrogen fill port to enable sample freezing to $77 \mathrm{~K}$ within the glovebox. Each sample was loaded into a Delrin Mössbauer sample cup for measurements and loaded under liquid nitrogen. Low-temperature ${ }^{57} \mathrm{Fe}$ Mössbauer measurements were performed using a SeeCo. MS4 Mössbauer spectrometer integrated with a Janis SVT-400T He/ $\mathrm{N}_{2}$ cryostat for measurements at $5 \mathrm{~K}$ to $130 \mathrm{~K}$ with a $0.07 \mathrm{~T}$ applied magnetic field. Isomer shifts were determined relative to $\alpha$-Fe at $298 \mathrm{~K}$. All Mössbauer spectra were fit using the program WMoss (SeeCo). Errors of the fit analyses were the following: $\delta \pm 0.02 \mathrm{~mm} / \mathrm{s}$ and $\Delta \mathrm{E}_{\mathrm{Q}} \pm 3 \%$. For multicomponent fits the quantitation errors were $\pm 3 \%$ (e.g., $70 \pm 3 \%$ ).

Computational Methods. The geometries were initially optimized at the density functional theory (DFT) level with Perdew, Burke and Ernzerhof's 1996 gradient-corrected exchangecorrelation functional (PBE), ${ }^{1,2}$ and TZVP basis set $^{3}$ for all atoms, with and without the empirical dispersion correction of Grimme (DG3) ${ }^{4}$ using Gaussian09 program system. ${ }^{5}$ Harmonic frequency calculations were used to confirm that the optimized structures were energy minima. The Cartesian coordinates for the optimized geometries are given in Supporting Information. The electronic structure descriptors (such as molecular orbital compositions and Mayer two-center bond orders, ${ }^{6}$ their alpha- and beta-spin components, ${ }^{7}$ and atomic spin densities were calculated using the AOMix software ${ }^{8,9}$ from the Gaussian 09-optimized wave functions.

Crystallographic Methods. Data collection results for compounds 1, 2, 3, and 4a represent the best data sets obtained in several trials for each sample. The crystals were mounted on thin glass fibers using paraffin oil. Prior to data collection crystals were cooled to $200 \mathrm{~K}$. Data were collected on a Bruker AXS SMART single crystal diffractometer equipped with a sealed Mo tube source (wavelength $0.71073 \AA$ ) APEX II CCD detector. Raw data collection and processing were performed with APEX II software package from BRUKER AXS. ${ }^{10}$ Diffraction data for samples 2 and $\mathbf{3}$ were collected with a sequence of $0.3^{\circ} \omega$ scans at 0,120 , and $240^{\circ}$ in $\varphi$. Due to lower unit cell symmetry in order to ensure adequate data redundancy, diffraction data for $\mathbf{1}$ and $4 \mathbf{a}$ were collected with a sequence of $0.3^{\circ} \omega$ scans at $0,90,180$ and $270^{\circ}$ in $\varphi$. Initial unit cell parameters were determined from 60 data frames with $0.3^{\circ} \omega$ scan each collected at the different sections of the Ewald sphere. Semi-empirical absorption corrections based on equivalent reflections were applied. ${ }^{11}$ Systematic absences in the diffraction data-set and unit-cell parameters were consistent with triclinic $\mathbf{P} \overline{1}$ (№2) for compounds $\mathbf{1}$ and $\mathbf{4 a}$, monoclinic $\mathbf{P} 2{ }_{1} / c$ (№14) for compound 2 and monoclinic $\mathbf{P} 2_{1} / n$ (№14, alternative settings) for compounds 3. Solutions in the centrosymmetric space groups for all compounds yielded chemically reasonable and computationally stable results of refinement. The structures were solved by direct methods, completed with difference Fourier synthesis, and refined with full-matrix least-squares procedures based on $F^{2}$. Diffraction data for the crystal of complex 1 were collected to $0.75 \AA$ resolution, however due to small crystal size and weak diffraction it was discovered that both $\mathrm{R}$ (int) and $\mathrm{R}$ (sigma) exceed $35 \%$ for the data below $1.00 \AA$ resolution. Based on $\mathrm{R}$ (sigma) value, data were truncated to $0.95 \AA$ resolution for refinement. The structural model of $\mathbf{1}$ contains one trinuclear iron compound molecule, two $\mathrm{CF}_{3} \mathrm{SO}_{3}{ }^{-}$anions and two chloroform molecules all located in general positions of the unit cell. To ensure proper geometry of the fragments and 
adequate values for thermal motion parameters, a set of geometry constraints (SAME) and thermal motion restraints (SIMU, DELU) were applied to both $\mathrm{CF}_{3} \mathrm{SO}_{3}{ }^{-}$moieties. After application of anisotropic thermal displacement parameters for all non-hydrogen atoms in the structural model it was discovered that one of the chloroform molecule was shift-disordered by a non-symmetry-related shift. To model this disorder the chlorine atoms with excessively large thermal motion parameters were split over two positions and occupancies of those positions were allowed to refine. On the latest stages of refinement occupancies were fixed to $60 \%: 40 \%$ and a set of geometry restraints (SADI) were used to preserve reasonable geometry of the chloroform solvent molecules. The asymmetric unit for the structure of compound $\mathbf{2}$ reveals one dimeric iron fragment located on the inversion center of the unit cell, one $\mathrm{CF}_{3} \mathrm{SO}_{3}{ }^{-}$anion located in a general position and two molecules of acetonitrile solvent also located in the general positions. Initial refinement results for compound 3 suggested the presence of two non-merohedrally twinned domains. Two independent orientation matrices were found using CELL_NOW software. ${ }^{12}$ The data set was re-integrated with two independent orientation matrices and consecutive model refinement was performed using HKLF5 reflection data file. Twinning domain ratio coefficient (BASF) was refined to 0.45287. The structure of compound 3 consists of one dimeric ironcontaining molecule located on the inversion center of the unit cell. In addition, one $\mathrm{CF}_{3} \mathrm{SO}_{3}{ }^{-}$ anion and one molecule of acetonitrile solvent were located in general positions. To enforce acceptable anisotropic thermal motion parameters for non-hydrogen atoms of the acetonitrile fragment a set of thermal motion restraints (SIMU, DELU) were applied. Diffraction data for compound $4 \mathbf{a}$ were collected to $0.75 \AA$ resolution, however due to small crystal size and weak diffraction it was discovered that both $\mathrm{R}$ (int) and $\mathrm{R}$ (sigma) exceeded $35 \%$ for the data below $1.00 \AA$ resolution. Based on $\mathrm{R}($ sigma) value, data were truncated to $0.95 \AA$ resolution for refinement. The asymmetric unit of $\mathbf{4 a}$ contains one dimeric molecule located on the inversion center and one $\mathrm{CF}_{3} \mathrm{SO}_{3}{ }^{-}$anion located in the general position of the unit cell. In order to achieve an acceptable data to parameter ratio and obtain acceptable values for anisotropic displacement coefficients, a set of thermal motion restraints (SIMU, DELU) was applied to the whole structural model. For all the compounds all hydrogen atomic positions were calculated based on the geometry of the bearing non-hydrogen atoms. All hydrogen atoms were treated as idealized contributions during the refinement. All scattering factors are contained in several versions of the SHELXTL program library, with the latest version used being v.6.12. ${ }^{13}$ 
Optimized Cartesian coordinates $(\AA)$ of 1 from the PBE/TZVP calculations without a dispersion correction
$\mathrm{Fe} \quad-3.208547 \quad-0.008919 \quad-0.061196$
$\mathrm{Fe} \quad 3.209768 \quad 0.029803 \quad-0.143888$
Fe $\quad 0.012717 \quad 0.333997 \quad-0.215938$
S $\quad-1.752852 \quad 1.779193 \quad 0.318230$
S $\quad-4.765228-1.662034-0.481607$
S $\quad-1.547936-1.099696-1.292138$
S $\quad-4.742760 \quad 0.921376 \quad 1.376217$
S $\quad 1.736919 \quad 0.945535 \quad-1.699379$
S $\quad 4.739860 \quad-0.933534 \quad 1.262295$
S $\quad 1.556063 \quad-0.190094 \quad 1.475557$
$\begin{array}{llll}\text { S } & 4.805577 & 0.247514 & -1.789366\end{array}$
$\begin{array}{llll}\mathrm{N} & -3.797291 & 1.009829 & -1.634741\end{array}$
$\begin{array}{llll}\mathrm{N} & -2.554474 & -1.129068 & 1.437284\end{array}$
N $\quad 2.711711 \quad-1.717181-0.900060$
$\begin{array}{llll}\mathrm{N} & 3.541037 & 1.829540 & 0.590864\end{array}$
$\begin{array}{llll}\text { C } & -1.841631 & 2.422494 & -1.348994\end{array}$
$\begin{array}{llll}\text { C } & -2.822427 & 1.893964 & -2.208687\end{array}$
C $\quad-2.827000 \quad 2.273438 \quad-3.562881$
H $\quad-3.515358 \quad 1.798983 \quad-4.264403$
C $\quad-1.930185 \quad 3.235003 \quad-4.023884$
H $\quad-1.938446 \quad 3.518826 \quad-5.077184$
C $\quad-1.021348 \quad 3.830711 \quad-3.139863$
H $\quad-0.334546 \quad 4.600104 \quad-3.495901$ 

$\begin{array}{llll}\text { C } & -0.971636 & 3.420002 & -1.809024\end{array}$
H $\quad-0.239366 \quad 3.849372-1.123195$
$\begin{array}{llll}\text { C } & -4.993942 & 1.067980 & -2.182284\end{array}$
$\begin{array}{llll}\mathrm{H} & -5.145430 & 1.821125 & -2.963306\end{array}$
$\begin{array}{llll}\text { C } & -6.262614 & -0.808522 & -1.010115\end{array}$
$\begin{array}{llll}\text { C } & -6.196963 & 0.346401 & -1.823764\end{array}$
$\begin{array}{llll}\text { C } & -7.416731 & 0.868249 & -2.318591\end{array}$
$\begin{array}{llll}\text { H } & -7.389090 & 1.747619 & -2.966818\end{array}$
$\begin{array}{llll}\text { C } & -8.640502 & 0.295016 & -1.986072\end{array}$
$\begin{array}{llll}\text { H } & -9.564717 & 0.723467 & -2.376214\end{array}$
$\begin{array}{llll}\text { C } & -8.681350 & -0.827980 & -1.154530\end{array}$
$\begin{array}{llll}\mathrm{H} & -9.636516 & -1.280126 & -0.883478\end{array}$
$\begin{array}{llll}\text { C } & -7.488729 & -1.384332 & -0.678200\end{array}$
$\begin{array}{llll}\mathrm{H} & -7.510103 & -2.274908 & -0.047549\end{array}$
C $\quad-4.445891 \quad-2.587655-2.045314$
$\begin{array}{llll}\mathrm{H} & -5.347388 & -3.168682 & -2.272584\end{array}$

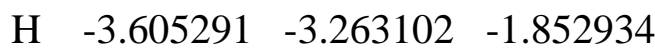
$\begin{array}{llll}\mathrm{H} & -4.201168 & -1.898751 & -2.860897\end{array}$
$\begin{array}{llll}\text { C } & -1.503220 & -2.537418 & -0.239772\end{array}$
$\begin{array}{llll}\text { C } & -2.076573 & -2.423907 & 1.041550\end{array}$
$\begin{array}{llll}\text { C } & -2.189166 & -3.565062 & 1.851051\end{array}$
H $\quad-2.713162 \quad-3.509466 \quad 2.806733$

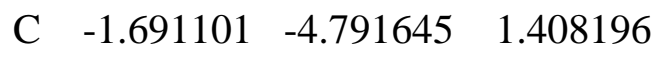
H $\quad-1.810313 \quad-5.677882 \quad 2.032896$
$\begin{array}{llll}\text { C } & -1.082561 & -4.890900 & 0.152348\end{array}$ 


$$
\begin{array}{llll}
\mathrm{H} & -0.717245 & -5.856123 & -0.203791 \\
\mathrm{C} & -0.991227 & -3.766512 & -0.672669 \\
\mathrm{H} & -0.559662 & -3.841986 & -1.673162 \\
\mathrm{C} & -2.476474 & -0.862158 & 2.720102 \\
\mathrm{H} & -1.940535 & -1.583794 & 3.347427 \\
\mathrm{C} & -3.914972 & 1.235265 & 2.947450 \\
\mathrm{C} & -2.977313 & 0.289783 & 3.434939 \\
\mathrm{C} & -2.492965 & 0.454635 & 4.755453 \\
\mathrm{H} & -1.793052 & -0.287677 & 5.147353 \\
\mathrm{C} & -2.879710 & 1.526007 & 5.550690 \\
\mathrm{H} & -2.486697 & 1.629307 & 6.562781 \\
\mathrm{C} & -3.792001 & 2.456397 & 5.045165 \\
\mathrm{H} & -4.116543 & 3.300463 & 5.655800 \\
\mathrm{C} & -4.320392 & 2.296433 & 3.761171 \\
\mathrm{H} & -5.057122 & 3.016328 & 3.408442 \\
\mathrm{C} & -5.212412 & 2.619427 & 0.870633 \\
\mathrm{H} & -6.013858 & 2.986233 & 1.520911 \\
\mathrm{H} & -5.603608 & 2.531145 & -0.147769 \\
\mathrm{H} & -4.335948 & 3.277568 & 0.888460 \\
\mathrm{C} & 1.723286 & -0.487562 & -2.760289 \\
\mathrm{C} & 2.247201 & -1.695904 & -2.259619 \\
\mathrm{C} & 2.330961 & -2.812289 & -3.112529 \\
\mathrm{H} & 2.817485 & -3.728907 & -2.773533 \\
\mathrm{H} & 1.843581 & -2.739907 & -4.416700 \\
\hline & 1.934008 & -3.605232 & -5.074808
\end{array}
$$



C $\quad 1.275284 \quad-1.550953 \quad-4.889409$
H $\quad 0.903154 \quad-1.491075 \quad-5.913353$
$\begin{array}{llll}\text { C } & 1.219956 & -0.427586 & -4.064922\end{array}$
$\begin{array}{llll}\mathrm{H} & 0.808101 & 0.512580 & -4.435619\end{array}$
$\begin{array}{llll}\text { C } & 2.673288 & -2.881819 & -0.291006\end{array}$
H $\quad 2.150832 \quad-3.692522 \quad-0.809913$
$\begin{array}{llll}\text { C } & 4.122043 & -2.539943 & 1.801787\end{array}$
$\begin{array}{llll}\text { C } & 3.227826 & -3.277722 & 0.984941\end{array}$
$\begin{array}{llll}\text { C } & 2.870880 & -4.580577 & 1.416202\end{array}$
H $\quad 2.186187 \quad-5.159655 \quad 0.792541$
$\begin{array}{llll}\text { C } & 3.357913 & -5.128452 & 2.594671\end{array}$
H $3.056163 \quad-6.131122 \quad 2.899583$
$\begin{array}{llll}\text { C } & 4.253447 & -4.390286 & 3.373591\end{array}$
H $\quad 4.662096 \quad-4.808288 \quad 4.294820$
$\begin{array}{llll}\text { C } & 4.644411 & -3.113593 & 2.964494\end{array}$
$\begin{array}{llll}\mathrm{H} & 5.364757 & -2.567607 & 3.572606\end{array}$
$\begin{array}{llll}\text { C } & 4.832135 & -0.040993 & 2.864934\end{array}$
H $\quad 5.626077 \quad-0.475558 \quad 3.480724$
H $\quad 3.862722 \quad-0.075661 \quad 3.374214$
$\begin{array}{llll}\mathrm{H} & 5.104350 & 0.991427 & 2.622489\end{array}$
$\begin{array}{llll}\text { C } & 1.579124 & 1.515280 & 2.010927\end{array}$
$\begin{array}{llll}\text { C } & 2.511076 & 2.391835 & 1.420223\end{array}$
$\begin{array}{llll}\text { C } & 2.398496 & 3.772609 & 1.681101\end{array}$
H $3.021913 \quad 4.488841 \quad 1.144425$
$\begin{array}{llll}\text { C } & 1.465910 & 4.246165 & 2.599973\end{array}$ 

H $\quad 1.393067 \quad 5.318258 \quad 2.787760$
$\begin{array}{llll}\text { C } & 0.622504 & 3.352053 & 3.271172\end{array}$
$\begin{array}{llll}\mathrm{H} & -0.095424 & 3.718034 & 4.006778\end{array}$
$\begin{array}{llll}\text { C } & 0.670223 & 1.995089 & 2.964862\end{array}$
$\begin{array}{llll}\text { H } & -0.022249 & 1.300127 & 3.441979\end{array}$
$\begin{array}{llll}\text { C } & 4.640627 & 2.548222 & 0.504066\end{array}$
H $\quad 4.691900 \quad 3.436401 \quad 1.142336$
C $\quad 6.091842 \quad 1.395153 \quad-1.279926$
$\begin{array}{llll}\text { C } & 5.841297 & 2.367089 & -0.279297\end{array}$
$\begin{array}{llll}\text { C } & 6.870187 & 3.309084 & -0.009692\end{array}$
H $\quad 6.690483 \quad 4.068672 \quad 0.754856$
$\begin{array}{llll}\text { C } & 8.082528 & 3.291854 & -0.683079\end{array}$
H $\quad 8.852250 \quad 4.026614 \quad-0.444685$
$\begin{array}{llll}\text { C } & 8.299758 & 2.333225 & -1.678133\end{array}$
H $\quad 9.240129 \quad 2.312385 \quad-2.230936$
C $\quad 7.302111 \quad 1.406183 \quad-1.981693$
H $\quad 7.479573 \quad 0.688478 \quad-2.782460$
$\begin{array}{llll}\text { C } & 5.784259 & -1.303494 & -1.952709\end{array}$
$\begin{array}{llll}\mathrm{H} & 6.491141 & -1.198617 & -2.781844\end{array}$
$\begin{array}{llll}\mathrm{H} & 5.063428 & -2.088051 & -2.205812\end{array}$
H $\quad 6.302743 \quad-1.535718-1.015846$ 
Optimized Cartesian coordinates $(\AA)$ of $\mathbf{1}$ from the PBE/TZVP calculations with the empirical dispersion correction (DG3)
$\begin{array}{llll}\mathrm{Fe} & 3.088107 & 0.002045 & -0.044407\end{array}$
Fe $\quad-3.097429 \quad 0.045061 \quad-0.195204$
Fe $\quad-0.013102 \quad-0.284535 \quad-0.409712$
$\begin{array}{llll}\text { S } & 1.708241 & -1.833775 & -0.497960\end{array}$
S $\quad 4.638308 \quad 1.674405 \quad 0.292958$
$\begin{array}{llll}\text { S } & 1.494039 & 1.475083 & -0.914631\end{array}$
S $\quad 4.499945-1.393638 \quad 1.100521$
S $\quad-1.697431-0.238803-2.033603$
$\begin{array}{llll}\text { S } & -4.556846 & 0.496892 & 1.506312\end{array}$
$\begin{array}{llll}\text { S } & -1.480792 & -0.569814 & 1.373666\end{array}$
$\begin{array}{llll}\text { S } & -4.728484 & 0.584180 & -1.726209\end{array}$
N $3.811485 \quad-0.332561 \quad-1.833256$
$\begin{array}{llll}\mathrm{N} & 2.286155 & 0.508093 & 1.695363\end{array}$
N $\quad-2.459126 \quad 1.905558 \quad-0.175111$
N $\quad-3.543385 \quad-1.864199 \quad-0.299107$
C $\quad 1.843366 \quad-1.674738-2.275401$
C $\quad 2.864782 \quad-0.848262 \quad-2.777859$
C $\quad 2.920301 \quad-0.573509 \quad-4.153775$
H $\quad 3.650359 \quad 0.139640 \quad-4.540808$
C $\quad 2.016072 \quad-1.183486 \quad-5.022050$
H $\quad 2.057280 \quad-0.958537 \quad-6.088615$
C $\quad 1.055436 \quad-2.074681 \quad-4.526503$
H $\quad 0.358424 \quad-2.563176 \quad-5.209065$ 

$\begin{array}{llll}\text { C } & 0.964742 & -2.316619 & -3.156178\end{array}$
H $\quad 0.189689 \quad-2.972190 \quad-2.755659$
$\begin{array}{llll}\text { C } & 5.044562 & -0.207753 & -2.275189\end{array}$
H $\quad 5.252461 \quad-0.576721 \quad-3.285595$
$\begin{array}{llll}\text { C } & 6.181482 & 0.996529 & -0.344820\end{array}$
$\begin{array}{llll}\text { C } & 6.208399 & 0.250643 & -1.546600\end{array}$
$\begin{array}{llll}\text { C } & 7.473094 & -0.114932 & -2.063899\end{array}$
H $\quad 7.518731 \quad-0.671481 \quad-3.003183$
$\begin{array}{llll}\text { C } & 8.649577 & 0.200618 & -1.389642\end{array}$
H $\quad 9.611210 \quad-0.103703 \quad-1.804995$
$\begin{array}{llll}\text { C } & 8.596106 & 0.907064 & -0.184319\end{array}$
$\begin{array}{llll}\text { H } & 9.513537 & 1.153686 & 0.351993\end{array}$
$\begin{array}{llll}\text { C } & 7.359936 & 1.318647 & 0.327096\end{array}$
$\begin{array}{llll}\mathrm{H} & 7.310018 & 1.899605 & 1.249553\end{array}$
$\begin{array}{llll}\text { C } & 4.448928 & 3.052587 & -0.915029\end{array}$
$\begin{array}{llll}\text { H } & 5.358855 & 3.662116 & -0.868598\end{array}$
H $\quad 3.584047 \quad 3.641764 \quad-0.592191$
$\mathrm{H} \quad 4.284319 \quad 2.657784 \quad-1.923347$
$\begin{array}{llll}\text { C } & 1.411859 & 2.466029 & 0.561531\end{array}$
$\begin{array}{llll}\text { C } & 1.868290 & 1.879569 & 1.758986\end{array}$
$\begin{array}{llll}\text { C } & 1.953578 & 2.655755 & 2.922811\end{array}$
$\mathrm{H} \quad 2.383780 \quad 2.232210 \quad 3.831964$
C $\quad 1.560231 \quad 3.996355 \quad 2.900204$
H $\quad 1.664887 \quad 4.602924 \quad 3.800804$
$\begin{array}{llll}\text { C } & 1.079578 & 4.568338 & 1.717603\end{array}$ 

$\begin{array}{llll}\mathrm{H} & 0.797491 & 5.622813 & 1.694207\end{array}$
$\begin{array}{llll}\text { C } & 1.001242 & 3.803732 & 0.548758\end{array}$
$\begin{array}{llll}\mathrm{H} & 0.657531 & 4.246519 & -0.388496\end{array}$
$\begin{array}{llll}\text { C } & 2.077969 & -0.211244 & 2.772081\end{array}$
H $\quad 1.484469 \quad 0.242641 \quad 3.573695$
$\begin{array}{llll}\text { C } & 3.470188 & -2.290345 & 2.281341\end{array}$
$\begin{array}{llll}\text { C } & 2.490423 & -1.573905 & 3.016530\end{array}$
$\begin{array}{llll}\text { C } & 1.844906 & -2.236843 & 4.087149\end{array}$
H $\quad 1.106904 \quad-1.681967 \quad 4.671587$
$\begin{array}{llll}\text { C } & 2.109029 & -3.567181 & 4.390065\end{array}$
H $\quad 1.588217 \quad-4.056426 \quad 5.213846$
$\begin{array}{llll}\text { C } & 3.053286 & -4.265971 & 3.632635\end{array}$
H $\quad 3.276784 \quad-5.310481 \quad 3.855315$
$\begin{array}{llll}\text { C } & 3.744597 & -3.621750 & 2.602205\end{array}$
H $\quad 4.503079 \quad-4.179238 \quad 2.055279$
$\begin{array}{llll}\text { C } & 5.097049 & -2.749954 & 0.026347\end{array}$
H $\quad 5.822082 \quad-3.362591 \quad 0.573033$
H $\quad 5.609414 \quad-2.260324 \quad-0.807511$
H $\quad 4.248997 \quad-3.339885 \quad-0.341382$
$\begin{array}{llll}\text { C } & -1.610254 & 1.498476 & -2.420822\end{array}$
$\begin{array}{llll}\text { C } & -2.022605 & 2.424242 & -1.441083\end{array}$
$\begin{array}{llll}\text { C } & -2.011231 & 3.797196 & -1.743617\end{array}$
$\begin{array}{llll}\mathrm{H} & -2.407205 & 4.521215 & -1.028623\end{array}$
$\begin{array}{llll}\text { C } & -1.540573 & 4.239388 & -2.979768\end{array}$
H $\quad-1.556011 \quad 5.304956-3.212717$ 

$\begin{array}{llll}\text { C } & -1.083580 & 3.316916 & -3.928812\end{array}$
H $\quad-0.722236 \quad 3.663182-4.898338$
$\begin{array}{llll}\text { C } & -1.121733 & 1.950494 & -3.651561\end{array}$
$\begin{array}{llll}\mathrm{H} & -0.788618 & 1.222517 & -4.393387\end{array}$
$\begin{array}{llll}\text { C } & -2.298784 & 2.687033 & 0.868155\end{array}$
$\begin{array}{llll}\mathrm{H} & -1.723830 & 3.605629 & 0.718422\end{array}$

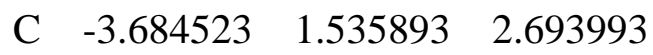
$\begin{array}{llll}\text { C } & -2.739825 & 2.487693 & 2.231352\end{array}$
$\begin{array}{llll}\text { C } & -2.176643 & 3.371314 & 3.183592\end{array}$
$\begin{array}{llll}\mathrm{H} & -1.449728 & 4.108123 & 2.836443\end{array}$
$\begin{array}{llll}\text { C } & -2.504660 & 3.307795 & 4.531487\end{array}$
$\begin{array}{llll}\mathrm{H} & -2.043579 & 3.996152 & 5.240747\end{array}$
$\begin{array}{llll}\text { C } & -3.442695 & 2.367319 & 4.964229\end{array}$
$\begin{array}{llll}\mathrm{H} & -3.725578 & 2.309740 & 6.016400\end{array}$
$\begin{array}{llll}\text { C } & -4.042041 & 1.503347 & 4.043475\end{array}$
$\begin{array}{llll}\mathrm{H} & -4.790921 & 0.797961 & 4.400827\end{array}$
$\begin{array}{llll}\text { C } & -4.871501 & -1.007853 & 2.504365\end{array}$
$\begin{array}{llll}\mathrm{H} & -5.622473 & -0.792972 & 3.271697\end{array}$
$\begin{array}{llll}\mathrm{H} & -3.934045 & -1.371269 & 2.940629\end{array}$
$\begin{array}{llll}\mathrm{H} & -5.282828 & -1.745609 & 1.808020\end{array}$
$\begin{array}{llll}\text { C } & -1.536854 & -2.320640 & 1.002387\end{array}$
$\begin{array}{llll}\text { C } & -2.502188 & -2.773722 & 0.082330\end{array}$
$\begin{array}{llll}\text { C } & -2.419300 & -4.092632 & -0.400665\end{array}$

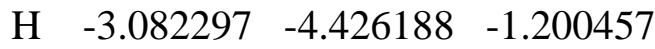
$\begin{array}{llll}\text { C } & -1.469940 & -4.972344 & 0.115148\end{array}$ 

H $\quad-1.412778 \quad-5.990378 \quad-0.272314$
C $\quad-0.592911-4.547896 \quad 1.120921$
H $\quad 0.139445 \quad-5.238236 \quad 1.542286$
C $\quad-0.618843 \quad-3.224003 \quad 1.552030$
H $\quad 0.107251 \quad-2.870443 \quad 2.283659$
C $\quad-4.693564-2.424461 \quad-0.601606$
H $\quad-4.764214 \quad-3.507992 \quad-0.458954$
C $\quad-6.146927 \quad-0.484139-1.440433$
C $\quad-5.939205 \quad-1.822130-1.018040$
C $\quad-7.066262-2.681602 \quad-0.967967$
H $\quad-6.915815 \quad-3.717609 \quad-0.655206$
C $\quad-8.342130 \quad-2.240797 \quad-1.290251$
H $\quad-9.189441 \quad-2.924749 \quad-1.232824$
C $\quad-8.525359-0.915802-1.696635$
H $\quad-9.518990 \quad-0.551028 \quad-1.961044$
C $\quad-7.431016 \quad-0.053068 \quad-1.787709$
H $\quad-7.596982 \quad 0.963885 \quad-2.139789$
C $\quad-5.449275 \quad 2.231424 \quad-1.346783$
H $\quad-6.159404 \quad 2.506176 \quad-2.133734$
H $\quad-4.609941 \quad 2.933456-1.374962$
H $\quad-5.924400 \quad 2.228171 \quad-0.359042$ 


\section{Optimized Cartesian coordinates ( $\mathrm{\AA}$ ) of 5 (at the PBEPBE/TZVP level)}
Fe $\quad-0.185100 \quad-0.187753 \quad 0.058807$
N $\quad 0.399311 \quad 1.749742 \quad 0.093828$
S $\quad 1.043941 \quad-0.637434 \quad-1.843611$
$\begin{array}{llll}\text { S } & -1.228741 & 0.288541 & 2.064819\end{array}$
C $\quad 3.612224 \quad-0.036653 \quad-2.558220$
C $\quad 4.757551 \quad 0.753333 \quad-2.688077$
C $\quad 0.396785 \quad-0.053888 \quad-3.475899$
C $\quad 2.504476 \quad 0.425265 \quad-1.843362$
C $\quad 4.791668 \quad 2.022626 \quad-2.101494$
C $\quad 3.690667 \quad 2.481832-1.388283$
C $\quad 2.522887 \quad 1.694901 \quad-1.227835$
C $\quad 1.473510 \quad 2.288186 \quad-0.425814$
C $\quad-0.417514 \quad 2.621685 \quad 0.882234$
C $\quad-1.154364 \quad 2.035607 \quad 1.934851$
$\begin{array}{llll}\text { C } & -0.481257 & 4.014045 & 0.677989\end{array}$
$\begin{array}{llll}\text { C } & -1.832018 & 2.873086 & 2.844585\end{array}$
$\begin{array}{llll}\text { C } & -1.193372 & 4.823469 & 1.556190\end{array}$
$\begin{array}{llll}\text { C } & -1.846790 & 4.250581 & 2.660483\end{array}$
H $\quad 3.574462 \quad-1.026963 \quad-3.016951$
$\begin{array}{llll}\mathrm{H} & 5.616924 & 0.377378 & -3.245571\end{array}$ 


$$
\begin{aligned}
& \text { H } \quad 1.196294 \quad-0.219553 \quad-4.207350 \\
& \text { H } \quad-0.478478 \quad-0.666957 \quad-3.707591 \\
& \mathrm{H} \quad 5.676884 \quad 2.652322 \quad-2.201496 \\
& \begin{array}{llll}
\mathrm{H} & 0.122700 & 1.005285 & -3.425606
\end{array} \\
& \text { H } \quad 3.719293 \quad 3.472191 \quad-0.927614 \\
& \text { H } \quad 1.671401 \quad 3.335296 \quad-0.170623 \\
& \text { H } \quad-0.006962 \quad 4.461425 \quad-0.197106 \\
& \begin{array}{llll}
\mathrm{H} & -2.370124 & 2.420329 & 3.679720
\end{array} \\
& \begin{array}{llll}
\mathrm{H} & -1.255225 & 5.897396 & 1.375964
\end{array} \\
& \begin{array}{llll}
\mathrm{H} & -2.393358 & 4.883972 & 3.361492
\end{array} \\
& \begin{array}{llll}
\mathrm{P} & -0.962337 & -2.266853 & 0.098182
\end{array} \\
& \begin{array}{llll}
\mathrm{P} & 1.601197 & -0.727044 & 1.312199
\end{array} \\
& \begin{array}{llll}
\mathrm{P} & -2.027054 & 0.428046 & -1.125585
\end{array} \\
& \begin{array}{llll}
\text { O } & 2.727411 & -1.634832 & 0.510472
\end{array} \\
& \begin{array}{llll}
\text { O } & 1.253610 & -1.567877 & 2.687059
\end{array} \\
& \begin{array}{llll}
\text { O } & 2.621628 & 0.462815 & 1.860015
\end{array} \\
& \begin{array}{llll}
\text { O } & -0.685174 & -3.322694 & 1.346874
\end{array} \\
& \begin{array}{llll}
\text { O } & -2.601461 & -2.319628 & -0.066405
\end{array} \\
& \begin{array}{llll}
\mathrm{O} & -0.503430 & -3.261278 & -1.137587
\end{array} \\
& \begin{array}{llll}
\text { O } & -2.355747 & -0.565144 & -2.403615
\end{array} \\
& \begin{array}{llll}
O & -3.571122 & 0.556615 & -0.566899
\end{array}
\end{aligned}
$$




$$
\begin{aligned}
& \begin{array}{llll}
\mathrm{O} & -1.740443 & 1.908934 & -1.824812
\end{array} \\
& \begin{array}{llll}
\text { C } & -1.346876 & -3.178152 & 2.625591
\end{array} \\
& \text { H } \quad-1.209941 \quad-4.136301 \quad 3.141916 \\
& \begin{array}{llll}
\mathrm{H} & -0.887943 & -2.370358 & 3.208660
\end{array} \\
& \text { H } \quad-2.421196 \quad-2.981531 \quad 2.497134 \\
& \begin{array}{llll}
\text { C } & -3.277213 & -3.585040 & -0.278325
\end{array} \\
& \begin{array}{llll}
\mathrm{H} & -2.912483 & -4.356249 & 0.414667
\end{array} \\
& \begin{array}{llll}
\text { H } & -4.340326 & -3.393437 & -0.090757
\end{array}
\end{aligned}
$$

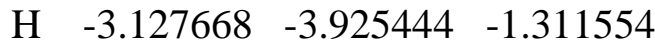

$$
\begin{aligned}
& \text { C } \quad 0.650810 \quad-4.130200 \quad-1.056432 \\
& \mathrm{H} \quad 0.513527 \quad-4.875847 \quad-0.262253 \\
& \text { H } \quad 0.713902 \quad-4.628106 \quad-2.031215
\end{aligned}
$$

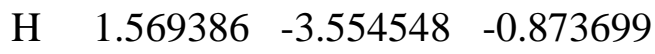

$$
\begin{aligned}
& \begin{array}{llll}
\text { C } & -3.681877 & -0.925700 & -2.853513
\end{array} \\
& \begin{array}{llll}
\mathrm{H} & -3.529380 & -1.649119 & -3.663737
\end{array} \\
& \text { H } \quad-4.254382 \quad-1.384682-2.038942 \\
& \text { H } \quad-4.228297 \quad-0.052716 \quad-3.236216 \\
& \begin{array}{llll}
\text { C } & -4.030631 & 1.539790 & 0.386659
\end{array} \\
& \begin{array}{llll}
\mathrm{H} & -3.494698 & 2.493790 & 0.281445
\end{array} \\
& \begin{array}{llll}
\mathrm{H} & -5.096738 & 1.690004 & 0.173036
\end{array} \\
& \begin{array}{llll}
\mathrm{H} & -3.899809 & 1.153432 & 1.404430
\end{array}
\end{aligned}
$$



$\begin{array}{llll}\text { C } & -2.654576 & 2.470914 & -2.793698\end{array}$
$\mathrm{H} \quad-2.577101 \quad 1.934417 \quad-3.751241$
$\begin{array}{llll}\mathrm{H} & -3.693762 & 2.440527 & -2.435398\end{array}$
$\mathrm{H} \quad-2.350400 \quad 3.514844 \quad-2.935439$
$\begin{array}{llll}\text { C } & 2.272724 & -2.247671 & 3.450062\end{array}$
H $\quad 1.781541 \quad-2.605376 \quad 4.362868$
$\mathrm{H} \quad 2.665740 \quad-3.107047 \quad 2.887859$
H $\quad 3.092369 \quad-1.566738 \quad 3.724642$
$\begin{array}{llll}\text { C } & 2.265954 & 1.325739 & 2.965882\end{array}$
H $\quad 1.730095 \quad 2.213078 \quad 2.602995$
H $\quad 1.638997 \quad 0.800110 \quad 3.699477$
H $\quad 3.210944 \quad 1.631839 \quad 3.431974$
$\begin{array}{llll}\text { C } & 4.161042 & -1.558172 & 0.709351\end{array}$
$\mathrm{H} \quad 4.441258 \quad-1.803643 \quad 1.742110$
H $\quad 4.591023 \quad-2.300971 \quad 0.026872$
$\begin{array}{llll}\mathrm{H} & 4.533629 & -0.557397 & 0.457663\end{array}$ 


\section{References}

1. Perdew, J. P.; Burke, K.; Ernzerhof, M., Phys. Rev. Lett. 1996, 77, 3865.

2. Perdew, J. P.; Burke, K.; Ernzerhof, M., Phys. Rev. Lett. 1997, 78, 1396.

3. Schaefer, A.; Huber, C.; Ahlrichs, R., J. Chem. Phys. 1994, 100, 5829.

4. Grimme, S., J. Comp. Chem. 2006, 27, 1787.

5. Frisch, M. J. T., G. W.; Schlegel, H. B.; Scuseria, G. E.; Robb, M. A.; Cheeseman, J. R.; Scalmani, G.; Barone, V.; Mennucci, B.; Petersson, G. A.;Nakatsuji, H.; Caricato, M.; Li, X.; Hratchian, H. P.; Izmaylov, A. F.; Bloino, J.; Zheng, G.; Sonnenberg, J. L.; Hada, M.; Ehara, M.; Toyota, K.; Fukuda, R.; Hasegawa, J.; Ishida, M.; Nakajima, T.; Honda, Y.; Kitao, O.; Nakai, H.; Vreven, T.; J. A. Montgomery, J.; Peralta, J. E.; Ogliaro, F.; Bearpark, M.; Heyd, J. J.; Brothers, E.; Kudin, K. N.; Staroverov, V. N.; Kobayashi, R.; Normand, J.; Raghavachari, K.; Rendell, A.; Burant, J. C.; Iyengar, S. S.; Tomasi, J.; Cossi, M.; Rega, N.; Millam, J. M.; Klene, M.; Knox, J. E.; Cross, J. B.; Bakken, V.; Adamo, C.; Jaramillo, J.; Gomperts, R.; Stratmann, R. E.; Yazyev, O.; Austin, A. J.; Cammi, R.; Pomelli, C.; Ochterski, J. W.; Martin, R. L.; Morokuma, K.; Zakrzewski, V. G.; Voth, G. A.; Salvador, P.; Dannenberg, J. J.; Dapprich, S.; Daniels, A. D.; Farkas, Ö.; Foresman, J. B.; Ortiz, J. V.; Cioslowski, J.; Fox, D. J., Gaussian 09, Revision D.01, Gaussian, Inc: Wallingford, CT, 2013.

6. Mayer, I., Int. J. Quantum Chem. 1986, 29, 73.

7. Gorelsky, S. I.; Basumallick, L.; Vura-Weis, J.; Sarangi, R.; Hedman, B.; Hodgson, K. O.; Fujisawa, K.; Solomon, E., Inorg. Chem. 2005, 44, 4947.

8. Gorelsky, S. I. AOMix: Program for Molecular Orbital Analysis, http://www.sg-chem.net/, version 6.88, 2015.

9. Gorelsky, S. I.; Lever, A. B. P., J. Organomet. Chem. 2001, 635, 187.

10. APEX Software Suite. Bruker AXS: Mdison, WI: 2005; Vol. 2010.

11. Blessing, R., Acta Cryst. 1995, A51, 33.

12. Sheldrick, Cell_Now. Bruker-AXS: Madison, WI: 2004.

13. Sheldrick, G. M., Acta Cryst. 2008, A64, 112. 